\title{
A new multiwavelength census of blazars ${ }^{\star}$
}

\author{
A. Paggi ${ }^{1,2}$, M. Bonato ${ }^{3,4}$, C. M. Raiteri ${ }^{1}$, M. Villata $^{1}$, G. De Zotti ${ }^{4}$, and M. I. Carnerero ${ }^{1}$ \\ 1 INAF, Osservatorio Astrofisico di Torino, Via Osservatorio 20, 10025 Pino Torinese, Italy \\ e-mail: alessandro.paggi@inaf.it \\ 2 INFN - Istituto Nazionale di Fisica Nucleare, Sezione di Torino, Via Pietro Giuria 1, 10125 Turin, Italy \\ 3 INAF, Istituto di Radioastronomia, Via Piero Gobetti 101, 40129 Bologna, Italy \\ ${ }^{4}$ INAF, Osservatorio Astronomico di Padova, Vicolo dell-Osservaorio 5, 35122 Padova, Italy \\ Received 16 May 2020 / Accepted 14 June 2020
}

\begin{abstract}
Context. Blazars are the rarest and most powerful active galactic nuclei. They play a crucial and expanding role in the multifrequency and multimessenger astrophysics of today. Dominating the high-energy extragalactic sky, they have been recently associated with high-energy astrophysical neutrinos and they may be among the accelerators of the ultra-high energy cosmic rays. Current blazar catalogs, however, are incomplete and they are depleted, in particular, at low Galactic latitudes.

Aims. Our study is aimed at augmenting the current blazar census, starting from a sample of ALMA calibrators that provides more homogeneous sky coverage, especially at low Galactic latitudes, to build a catalog of blazar candidates that can provide candidate counterparts to unassociated $\gamma$-ray sources and sources of high-energy neutrino emission or ultra-high energy cosmic rays.

Methods. Based on the ALMA Calibrator Catalog, we compiled a selection of 1580 ALMA Blazar Candidates (ABCs) for which we collected multiwavelength information, including Gaia photometric, parallax, and proper motion data, as well as SDSS and LAMOST photometric and spectral data, WISE photometric data, X-ray (Swift-XRT, Chandra-ACIS and XMM-Newton-EPIC) count-rates and spectra, and Fermi-LAT fluxes and spectral slopes. We also compared our ALMA Blazar Candidates with existing blazar catalogs, such as 4FGL, 3HSP, WIBRaLS2, and the KDEBLLACS.

Results. The ABC catalog fills in the gaps with regard to low Galactic latitude sources in current blazar catalogs. By cross-matching this catalog with Gaia DR2, SDSS DR12, LAMOST DR5, AllWISE, and 4FGL catalogs, we obtained 805, 295, 31, 1311, and 259 matches, respectively. ALMA Blazar Candidates are significantly dimmer than known blazars in the Gaia $g$ band, while the difference in the Gaia $b-r$ color between the two populations is less pronounced. Also, ABC sources appear bluer in SDSS than known blazars, although with low statistical significance. Most ABCs classified as QSO and BL Lac fall into the SDSS color regions of low redshift quasars, with some QSOs entering the regions of higher redshift quasars. We collected 110 optical spectra in SDSS DR12 and LAMOST DR5, which mostly classify the corresponding sources as QSO (98), while 12 sources turned out to be galactic objects. Regarding the WISE colors, we found that ABC sources are significantly bluer than known blazars in the $w 2-w 3$ and $w 3-w 4$ colors. In X-ray, we detected $173 \mathrm{ABCs}$ and we were able to extract X-ray spectra for 92 of them. Our sources are, on average, similar in the X-ray band to known blazars in terms of count rates and spectral slopes, implying that our sample covers the same region of the blazar parameter space in this band. A comparison of $\gamma$-ray properties shows that the ABCs are, on average, dimmer and that their $\gamma$-ray spectra are softer than known blazars, indicating a significant contribution on the part of FSRQ sources. Making use of WISE colors, we classified $715 \mathrm{ABCs}$ as candidate $\gamma$-ray blazar of different classes.

Conclusions. We built a new catalog of 1580 candidate blazars with a rich multiwavelength data-set, filling in the gaps for low Galactic latitude sources in current blazar catalogs. This will be particularly important for the identification of the source population of high-energy neutrinos or ultra-high energy cosmic rays, or to verify the Gaia optical reference frame. In addition, ALMA Blazar Candidates can be investigated both through optical spectroscopic observation campaigns or through repeated photometric observations for variability studies. In this context, the data collected by the upcoming LSST surveys will provide a key tool for investigating the possible blazar nature of these sources.
\end{abstract}

Key words. catalogs - galaxies: active

\section{Introduction}

Blazars are the rarest and most powerful active galactic nuclei Their emission is charcterized by variable, non-thermal radiation that extends over the entire electromagnetic spectrum, flat radio spectra, high and variable polarization, apparent superluminal motion, and high luminosities characterized by intense and rapid variability (e.g., Urry \& Padovani 1995; Giommi et al. 2013). These observational properties are generally interpreted in terms

* Full Tables 1, 2, 4, C.1 and C.2 are only available at the CDS via anonymous ftp to cdsarc.u-strasbg. fr $(130.79 .128 .5)$ or via http://cdsarc.u-strasbg.fr/viz-bin/cat/J/A+A/641/A62 of a relativistic jet aligned within a small angle to our line of sight (Blandford \& Rees 1978). Traditionally blazars have been classified in two main subclasses: BL Lac objects and flat spectrum radio quasars (FSRQs). The former exhibit featureless optical spectra, while the latter are characterized by strong quasar emission lines and higher radio polarization. More specifically, if the only spectral features observed are emission lines with a rest-frame equivalent width of $\mathrm{EW}<5 \AA$, the object is classified as a BL Lac (Stickel et al. 1991; Stocke \& Rector 1997), otherwise it is classified as a FSRQ (Laurent-Muehleisen et al. 1999). The blazar spectral energy distributions (SEDs) typically show two peaks: one in the range of infrared-soft X-rays due to 
synchrotron emission by highly relativistic electrons within the jet, and another one at hard X-ray or $\gamma$-ray energies. The latter is interpreted as inverse Compton upscattering by the electrons in the jet on the seed photons provided by the synchrotron emission (synchrotron self-Compton, SSC, see, e.g., Inoue \& Takahara 1996) which dominates the high energy output in BL Lacs. The possible addition of seed photons from outside the jets yields contributions to the non-thermal radiations in the form of external inverse Compton scattering (EC, see Dermer \& Schlickeiser 1993; Dermer et al. 2009) often dominating the $\gamma$-ray outputs in FSRQs (Aharonian et al. 2009; Ackermann et al. 2011).

Blazars play a crucial and expanding role in the multifrequency and multimessenger astrophysics of today. They dominate the high-energy (from $\mathrm{MeV}$ to $\mathrm{TeV}$ ) extragalactic sky (Massaro et al. 2015a; Di Mauro et al. 2018; Chang et al. 2019; Chiaro et al. 2019) and they have recently been associated with high-energy astrophysical neutrinos (IceCube Collaboration 2018; Garrappa et al. 2019). In turn, these neutrinos arise from interactions of ultra-high energy cosmic rays (UHECR; Sarazin et al. 2019) via charged pion decay. Thus, blazars jets may also be among the long-sought accelerators of the UHECR.

The 5th edition of the Roma-BZCAT Multifrequency Catalogue of Blazars (Roma-BZCAT, Massaro et al. 2015b) is the most comprehensive list of blazars confirmed by means of published spectra. This catalog contains 3561 entries and for each source it lists radio coordinates obtained from very-longbaseline interferometry measurements (VLBI, Titov \& Malkin 2009; Titov et al. 2011; Petrov \& Taylor 2011), augmented with the available multiwavelength information, such as optical magnitude from USNO B1 or SDSS DR10, radio flux density from NVSS (Condon et al. 1998), FIRST (White et al. 1997), SUMSS (Mauch et al. 2003), GB6 (Gregory et al. 1996), or PMN (Wright et al. 1994), the microwave flux density from Planck (Planck Collaboration XXVIII 2014), the soft X-ray flux from ROSAT archive or Swift-XRT catalogs, the hard X-ray flux from Palermo BAT Catalogue (Cusumano et al. 2010), the $\gamma$-ray flux from the first (1FGL, Abdo et al. 2010a), as well as the Second Fermi catalog (2FGL, Nolan et al. 2012) and the redshift. Confirmed blazars listed in the Roma-BZCAT are subdivided into:

- 1059 BZBs: BL Lac objects,

- 1909 BZQs: Flat spectrum radio quasars.

In addition, the 5th edition of Roma-BZCAT lists 92 BL Lac candidates, that is, sources classified as BL Lac in the literature, but without published optical spectra. In the following, we consider these sources as BZBs. The other kind of sources listed in Roma-BZCAT are:

- 274 BZGs: sources classified as BL Lacs in the literature but with a host galactic emission dominating the nuclear one,

- 227 BZUs: blazars of uncertain type.

Blazar catalogs that are as large and complete as possible are necessary to provide candidate counterparts to unassociated $\gamma$-ray sources that have been detected by the Fermi satellite (D'Abrusco et al. 2013; Massaro et al. 2013) and to sources of high-energy neutrino emission or UHECRs. In particular, a complete sky coverage of such catalogs is critically important in the case of rare events, such as detections of high-energy neutrinos. The identification of the neutrino-emitting source population requires to fully exploit the all-sky neutrino sample by stacking analyses towards candidates. To this end, we obviously need blazar catalogs that cover the whole sky.

The Atacama Large Millimeter Array (ALMA) calibrators are compact sources bright at $\mathrm{mm}$ and sub-mm wavelengths. They were primarily drawn from "seed" catalogs, such as those of the Very Long Array (VLA), the SubMillimeter Array (SMA), the Australia Telescope Compact Array (ATCA), and the Combined Radio All-Sky Targeted Eight-GHz Survey (CRATES) ${ }^{1}$. The ALMA Calibrator Catalogue (ACC; Bonato et al. 2019) contains 3364 sources, whose distribution in Galactic coordinates is presented in the left panel of Fig. 1. Because of the ALMA location in the Southern Hemisphere, its calibration sources have declination lower than $\sim+60^{\circ}$; this explains the "hole" centered at about $l=-120^{\circ}$ and $b=30^{\circ}$ in the left panel of the figure.

The distribution of the Galactic latitude of ACC sources is presented in the upper part of the right panel of the same figure. The Galactic north-south asymmetry in latitude distribution is evident, with $\sim 50 \%$ of the ACC sources having $b<-10^{\circ}$ and $\sim 35 \%$ having $b>10^{\circ}$. In particular, we note that we have $\sim 15 \%$ of the sources at low Galactic latitudes, that is, $|b|<10^{\circ}$. This is at variance with the sources listed in the Roma-BZCAT, whose Galactic latitude distribution is shown in the lower part of the right panel of Fig. 1. In this case we have $\sim 40 \%$ of the RomaBZCAT sources with $b<-10^{\circ}$ and $\sim 57 \%$ with $b>10^{\circ}$, while only $\sim 3 \%$ of the Roma-BZCAT sources have $|b|<10^{\circ}$. This is mainly due to the fact that the firm blazar classification adopted in the Roma-BZCAT catalog is based on optical spectroscopy, which tends to be problematic along the Galactic plane due to strong absorption and source crowding.

The ALMA Calibrator Catalogue therefore represents an excellent starting point from which we can build a new catalog of blazar candidates, with the goal of completing the census of blazars, especially at low galactic latitudes where the current blazar catalogs are depleted.

In this paper, following an analysis of the sample of ALMA calibrators to select bona fide blazar candidates in Sect. 2, we compare the sample of selected sources, compiled into the ALMA Blazar Candidates (ABC) catalog, with other blazar catalogs in Sect. 3. In Sect. 4, we collect multiwavelength data on ABC sources by cross-matching our catalog with public infrared, optical, and $\gamma$-ray catalogs, and by performing an extensive X-ray analysis of available data. In Sect. 5, following D' Abrusco et al. (2019), we use data from the Wide-Field Infrared Survey Explorer (WISE, Wright et al. 2010) to select ABC sources whose midinfrared colors are consistent with those of confirmed $\gamma$-ray emitting blazars and to assign them to blazar sub-classes. Finally, in Sect. 6, we summarize our conclusions.

\section{Sample selection}

In this section, we compare the radio properties of ACC sources with those of Roma-BZCAT source and compile a sample of ACC sources that are not included in Roma-BZCAT in order to build a catalog of ALMA blazar candidates.

As mentioned before, the ACC contains 3364 sources, with a declination of $\left(\delta<60^{\circ}\right)$. The sources in Roma-BZCAT below such a declination are 3340. Cross-matching them with the ACC using a search radius of $10^{\prime \prime}$ we find 1391 matches. The comparison between the radio flux densities at 1.4 or $0.843 \mathrm{GHz}$ (as listed in the Roma-BZCAT) for these 1391 matches and the remaining 1949 Roma-BZCAT sources with declination $<60^{\circ}$ without a match in the ACC catalog is presented in the left panel of Fig. 2, from which it is evident that the ACC sources represent the brightest radio end of the blazar population. A similar result is found when comparing the average ALMA band 3 (65-90) GHz

\footnotetext{
https://almascience. eso.org/alma-data/calibratorcatalogue
} 

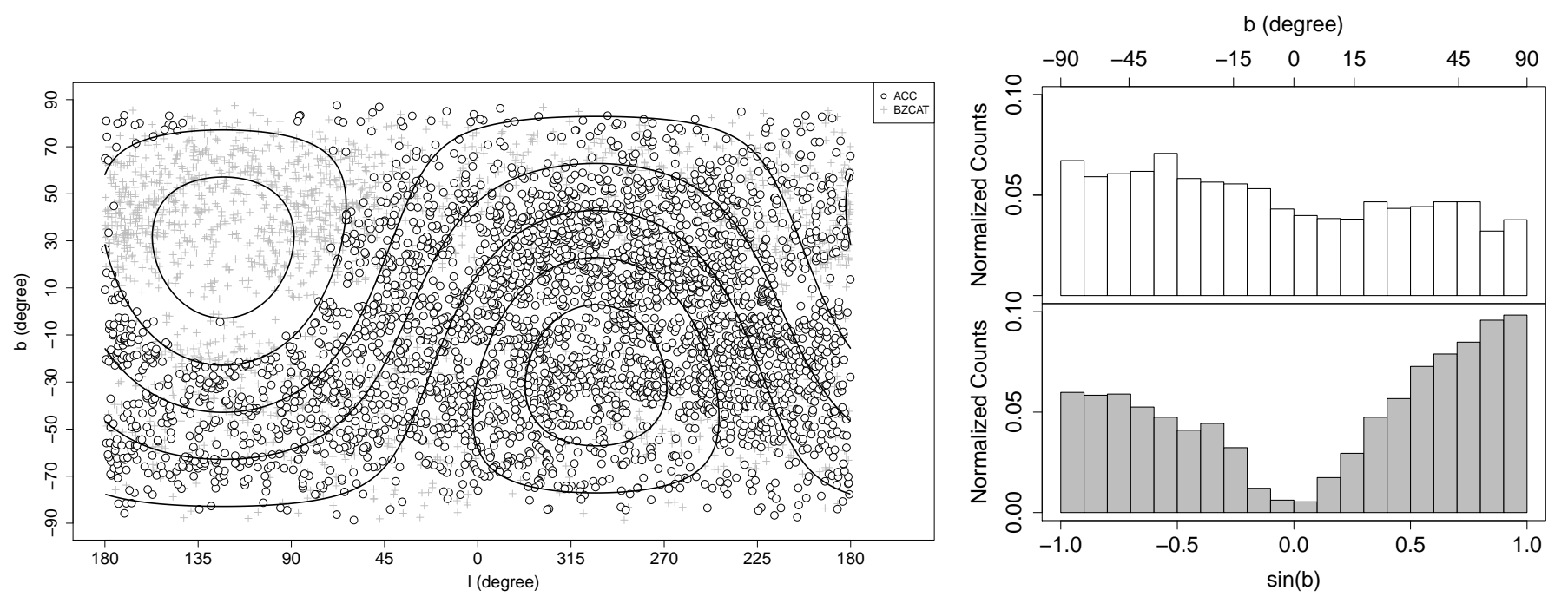

Fig. 1. Left panel: white circles indicate the Galactic coordinates of the ACC sources. Grey crosses in the background represent the Galactic coordinates of the Roma-BZCAT sources. Black lines represent equatorial coordinates with declinations from $-60^{\circ}$ to $60^{\circ}$ with increment of $20^{\circ}$. Right panel: normalized Galactic latitude distribution of ACC (top) and Roma-BZCAT (bottom) source.
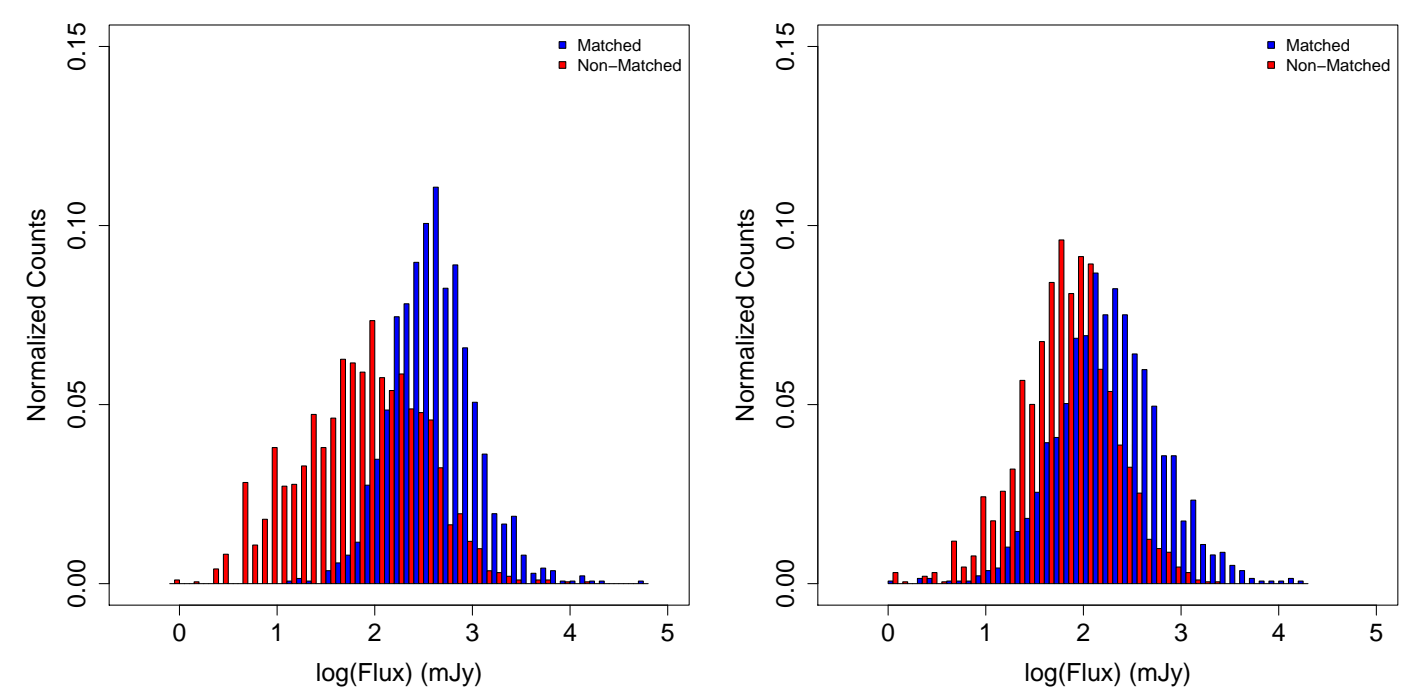

Fig. 2. Left panel: comparison of the normalized distributions of the radio fluxes densities at 1.4 or $0.843 \mathrm{GHz}$ (as listed in the Roma-BZCAT) for the Roma-BZCAT sources with a match in the ACC catalog (blue) and for the Roma-BZCAT sources below $<60^{\circ}$ of declination without a match in the ACC catalog (red). Right panel: comparison of the normalized distributions of the average ALMA band 3 radio fluxes densities for the ACC sources with a match in the Roma-BZCAT (blue) and for the ACC sources without a match in the Roma-BZCAT (red).

flux density of 1391 ACC sources that have a match with RomaBZCAT, with the remaining 1973 ACC sources without a RomaBZCAT match, as shown in the right panel of Fig. 2.

Then we excluded the 1391 sources with a match in the Roma-BZCAT from the sample of 3364 ACC sources, narrowing our selection down to 1973 sources. For the sources in ACC Bonato et al. (2019), the authors evaluated the low frequency spectral index, $\alpha_{\text {low }}$, between 1 and $5 \mathrm{GHz}$ using the $1.4 \mathrm{GHz}$ flux densities from NVSS, SUMSS, GB6, and PMN survey catalogs. We then considered only the sources classified in the ACC that are likely to be blazars, that is, sources with $\alpha_{\text {low }}<-0.5$ or demonstrating evidence of variability or $\gamma$-ray emission. This narrows our sample to 1646 sources. To get a preliminary characterization of these sources, we searched in the SIMBAD ${ }^{2}$ database (Wenger et al. 2000) for information from the literature and collected it in Table 1. In this table, in addition to the

$\overline{2 \text { http://simbad.u-strasbg.fr/simbad/ }}$ source name (Col. 1) and coordinates (Cols. 2 and 3), we list the source alternate name (Col. 4), the redshift (Col. 5), the source class given in literature according to SIMBAD object classification (Col. 6; see Table 1 note for more details), and the relative reference (Col. 7). We note that some sources are listed in the SIMBAD as "candidates" (AGN candidates: 2 sources; BL Lac candidates: 1 source; blazar candidates: 169 sources; quasar candidates: 3 sources). For the sake of simplicity, in the following, we consider candidates along with their respective sources that have a secure classification.

The composition of this sample of 1646 sources in terms of source class is presented in Fig. 3. It is evident that the three main contributors to the sample are generic radio sources (RS, $43.1 \%$ ), quasars (QSO, 26.3\%), and blazars (11.7\%).

Since we are interested in selecting a sample of blazar candidates, in the following, we exclude from our analysis the sources classified as galaxies (labeled as "G" in Fig. 3), galaxies in clusters, planetary nebulae, Seyfert galaxies, stars, etc. (labeled as 
Table 1. ALMA sources with flat radio spectrum without counterparts in the Roma-BZCAT (see Sect. 2).

\begin{tabular}{|c|c|c|c|c|c|c|}
\hline Name ALMA & RA & Dec & Name NED & Redshift & Object type & Reference \\
\hline J0002-2153 & 00:02:11.98 & $-21: 53: 09.87$ & WISEA J000211.98-215310.0 & & BLL & D'Abrusco et al. (2014) \\
\hline J0003-1941 & 00:03:15.95 & $-19: 41: 50.40$ & WISEA J000315.94-194150.1 & & QSO & Mignard et al. (2016) \\
\hline J0004-4345 & 00:04:07.26 & $-43: 45: 10.15$ & PKS 0001-440 & & $\mathrm{rG}$ & Titov et al. (2017) \\
\hline J0006-2955 & 00:06:01.12 & $-29: 55: 50.10$ & PKS 0003-302 & & QSO & Mignard et al. (2016) \\
\hline $\mathrm{J} 0008+1144$ & 00:08:00.84 & $+11: 44: 00.77$ & WISEA J000800.80+114400.9 & & $\operatorname{Rad}$ & \\
\hline J0008-1329 & 00:08:28.02 & $-13: 29: 30.57$ & PMN J0008-1329 & & $\operatorname{Rad}$ & \\
\hline J0008-3945 & 00:08:09.20 & $-39: 45: 22.96$ & WISE J000809.17-394522.8 & & $\mathrm{Rad}$ & \\
\hline J0010-0433 & 00:10:00.39 & $-04: 33: 48.51$ & WISEA J001000.38-043348.4 & & $\operatorname{Rad}$ & \\
\hline $\mathrm{J} 0011+0823$ & $00: 11: 35.27$ & $+08: 23: 55.59$ & WISEA J001 135.27+082355.5 & & QSO & Mignard et al. (2016) \\
\hline J0011-1434 & $00: 11: 40.46$ & $-14: 34: 04.63$ & LQAC 002-014 001 & & QSO & Mignard et al. (2016) \\
\hline
\end{tabular}

Notes. For each source we present the source name (Name ALMA), coordinates (RA and Dec), the source alternate name (Name NED), the redshift (Redshift), the source type given in literature (Object type, see note for more details) and the reference (Reference). SIMBAD object classification. AGN: Active Galaxy Nucleus, AG?: Possible Active Galaxy Nucleus, BCG: Brightest galaxy in a Cluster, BLL: BL Lac object, BL?: BL Lac candidate, Bla: Blazar, Bz?: Blazar candidate, G: Galaxy, gam: $\gamma$-ray source, GiC: Galaxy in cluster of galaxies, IR: infrared source, PaG: Pair of galaxies, PN: Planetary nebula, pr*: Pre-main sequence star, QSO: Quasar, Q?: Quasar candidate, Rad: Radio source, rG: Radio Galaxy, Sy1: Seyfert 1 galaxy, Sy2: Seyfert 2 galaxy, SyG: Seyfert galaxy, X: X-ray source. The full table is available in electronic form at the CDS.

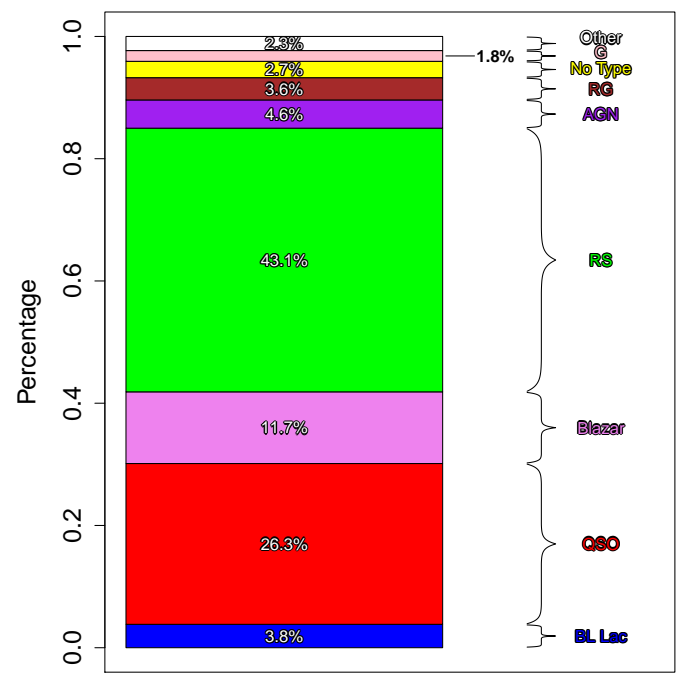

Fig. 3. Composition of the sample of 1646 ALMA calibrators with a flat radio spectrum and without a Roma-BZCAT counterpart in terms of their SIMBAD type. In blue: we present BL Lacs, in red: quasars (QSO), in violet: blazars, in green: radio sources (RS), in purple: active galactic nuclei (AGN), in brown: radio galaxies (RG), in yellow: sources without classification (No Type), in pink: galaxies (G), and in white: other type of sources (including galaxies in clusters, planetary nebulae, Seyfert galaxies, stars, etc).

"Other" in Fig. 3). In addition, we also include the only source with a $\gamma$-ray source classification (GAM in SIMBAD), namely J0055-1217, since its position at high Galactic latitude $\sim-75^{\circ}$ indicates that it is likely to be a blazar. As this is only one source, in the following, we include it in the RS group. This narrows our sample to 1580 sources, which represents about $\sim 96 \%$ of the ACC sample, referred to ALMA Blazar Candidates (ABC).

In Fig. 4, we compare the redshift distribution of sources in Roma-BZCAT (top panel) and ABC (lower panel). We note that redshift estimates are available for $706 \mathrm{ABC}$ sources $(\sim 46 \%)$ and for 2566 Roma-BZCAT sources $(\sim 72 \%)^{3}$. We see that BZQ 3 We exclude from this analysis the redshift estimate $z=6.802$ of
Roma-BZCAT source 5BZQJ1556+3517, since its SDSS spectrum

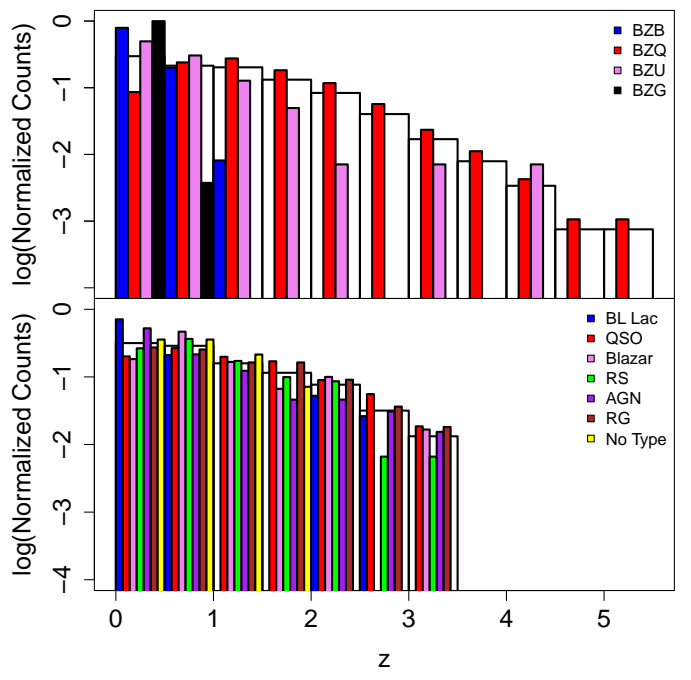

Fig. 4. Upper panel: redshift distribution of sources. The colored bars represent the different source classes indicated in the legend, while the white bars in the background show the redshift distribution of the whole Roma-BZCAT catalog. Lower panel: same as the upper panel, but for $\mathrm{ABC}$ sources.

sources extend up to $z \sim 5.5$, with BZG and BZB showing smaller redshifts $z \leq 0.6$ and $z \leq 1.3$, respectively. On the other hand, ABC sources only reach $z \sim 3.5$, with BL Lacs extending up to $z \leq 2.6$, and sources without classification reaching $z \leq 1.8$, while the other source types extend up to $z \leq 3.5$.

\section{Comparisons with other blazar candidate catalogs}

In this section, we compare the $\mathrm{ABC}$ catalog with other cata$\operatorname{logs}$ of blazar sources. The full results of this comparison are presented in Table C.1.

We start from the Fermi Large Area Telescope Fourth Source Catalog (4FGL, Abdollahi et al. 2020), the most recent release

shows that this estimate based on tentative detections of Ly- $\alpha$ and NV $\lambda 1240$ lines is doubtful. 

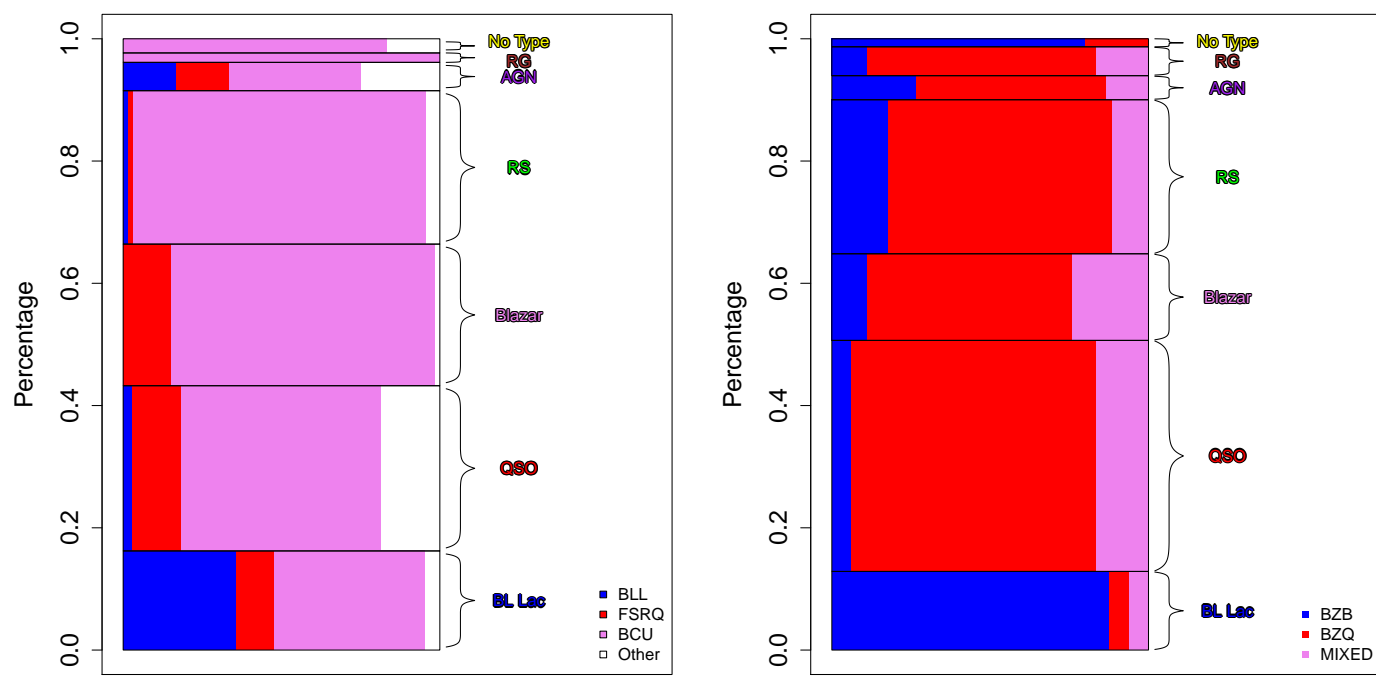

Fig. 5. Left panel: distribution of the 4FGL (Abdollahi et al. 2020) source types in the ABC source classes. Blue rectangles indicate BLLs, red rectangles indicate FSRQs, violet rectangles indicate BCUs, and white rectangles indicate other kind of sources (including compact steep spectrum radio sources, radio galaxies, starburst galaxies, generic active galactic nuclei, or unknown sources). Right panel: distribution of the WIBRaLS2 (D'Abrusco et al. 2019) source types in the ABC source classes. Blue rectangles indicate BZBs, red rectangles indicate BZQs and violet rectangles indicate MIXED sources.

of the Fermi mission $\gamma$-ray source catalog. Blazars represent the largest population of identified $\gamma$-ray sources. The 4FGL contains 5065 sources in the $50 \mathrm{MeV}-1 \mathrm{TeV}$ energy band, including 3915 sources associated with lower-energy counterparts with the Bayesian source association method (Abdo et al. 2010a) and the Likelihood Ratio method (Ackermann et al. 2011, 2015), both based on the spatial coincidence between the $\gamma$-ray sources and their potential counterparts, and 358 sources identified basing on periodic variability, correlated variability at other wavelengths, or spatial morphology.

Sources in 4FGL are classified as BLL (BL Lac objects), FSRQs, BCU (blazar of uncertain type), RDG (radio galaxies), AGNs, etc., according to the properties of their counterpart at other wavelengths. In particular, 3137 sources in the 4FGL are classified as blazars, which therefore represents $\sim 62 \%$ of the whole $4 \mathrm{FGL}$ and $\sim 80 \%$ of the identified sources.

We therefore cross-matched the catalog of $\mathrm{ABC}$ with the associated or identified sources in the 4FGL, adopting a search radius of $33^{\prime \prime} 0$ around the coordinates of the associated counterparts, finding 259 matches. We then looked for $\mathrm{ABCs}$ that would fall into the $90 \%$ uncertainty ellipse of unassociated unidentified sources in the 4FGL and found no matches.

The distribution of these matches in the different source classes of $\mathrm{ABC}$ is shown in the left panel of Fig. 5. In this figure, each rectangle represents an $\mathrm{ABC}$ class and the colored bars inside it represent the distribution of sources in this class among the 4FGL types.

We see that all ABC types are dominated by Fermi BCUs, which represent most $(\sim 73 \%)$ of the 4 FGL sources matching $\mathrm{ABC}$ sources. On the other hand, the majority of sources classified as BLL in the 4FGL fall in the BL Lac class, while FSRQs mainly fall in QSO and blazar classes. In addition, we note that six AGN and four RDG sources fall in the QSO class, while the ABC AGN type contains two BLLs, two FSRQs, five BCUs, and three RDGs.

We then compared the catalog of ABC with the Third catalog of extreme and High-Synchrotron Peaked blazars (3HSP, Chang et al. 2019). This is a catalog containing 2013 highsynchrotron peaked blazars (HSPs), that is, BL Lacs with the synchrotron component of their SED peaking at frequencies larger than $10^{15} \mathrm{~Hz}$. They were selected through multiwavelength analysis and 657 of these sources also appear in the 5th edition of Roma-BZCAT

We note that the Galactic latitude distribution of sources in 3HSP is similar to that of Roma-BZCAT (see Fig. 1), that is, peaking outside of the Galactic plane $\left(|b|>10^{\circ}\right)$. By crossmatching the ABC and the 3HSP catalogs adopting a search radius of $3{ }^{\prime \prime} 0$ we find 9 matches. In particular, one object has no classification in the $\mathrm{ABC}$ catalog, one object is classified ad Radio Galaxy, and seven are classified as BL Lacs.

Next we considered two catalogs of blazar candidates presented in D'Abrusco et al. (2019), namely, the second WISE Blazar-like Radio-Loud Sources (WIBRaLS2) catalog and the KDEBLLACS. The WIBRaLS2 catalog includes 9541 blazar candidates selected on the basis of their radio-loudness and of their WISE colors. Candidates were required to be detected in all four WISE bands. Their radio-loudness was defined on the basis of the ratio between the mid-infrared $22 \mu \mathrm{m}$ flux density $S_{22 \mu \mathrm{m}}$ and the radio flux density $S_{\text {Radio }}$, defined as $q_{22}=$ $\log \left(S_{22 \mu \mathrm{m}} / S_{\text {Radio }}\right)$. Radio flux densities were taken from NVSS (Condon et al. 1998), FIRST (White et al. 1997) and SUMSS (Mauch et al. 2003).

D'Abrusco et al. (2012) discovered that blazar listed in the second edition of Roma-BZCAT (Massaro et al. 2009) and associated with $\gamma$-ray sources listed in the Fermi Large Area Telescope Second Source Catalog (2FGL, Nolan et al. 2012) occupy a specific region of the two-dimensional WISE color-color planes. D'Abrusco et al. (2019) defined the region of the threedimensional principal component space generated by the three independent WISE colors occupied by blazars listed in the 5th edition of Roma-BZCAT and associated with $\gamma$-ray sources listed in the Fermi Large Area Telescope Third Source Cata$\log$ (3FGL, Acero et al. 2015). The WIBRaLS2 catalog contains radio-loud sources located in this region. Such sources are classified as candidates BZB and BZQ depending on whether they are compatible with the region occupied by BL Lacs or FSRQs. In the cases where they are compatible with both, they are classified 
as MIXED candidates ${ }^{4}$. Finally, blazar candidates in WIBRaLS2 are assigned a class going from D to A depending on how compatible a source is with the respective blazar WISE color region.

By cross-matching the ABC catalog with WIBRaLS2, adopting a search radius of $3{ }^{\prime \prime} 0$, we find 381 matches. The distribution of WIBRaLS2 source types among the various ABC classes is presented in the right panel of Fig. 5. Again, each rectangle in this figure represents an $\mathrm{ABC}$ class and the colored bars inside it represent the amount of sources in this class falling the WIBRaLS2 class types, according to the color code indicated in the legend. BZB sources are found mostly among BL Lacs, while other classes are dominated by BZQs which represent $\sim 64 \%$ of the WIBRaLS2 sources associated with ABC catalog.

KDEBLLACS is a catalog of BL Lac candidates selected among radio-loud sources using criteria that are analogous to those of the WIBRaLS2 catalog, except for the requirement of detection taking place in only the first three WISE bands and, consequently, for redefining the radio-loudness on the basis of their $S_{12 \mu \mathrm{m}} 12 \mu \mathrm{m}$ flux density, that is, on the ratio $q_{12}=$ $\log \left(S_{12 \mu \mathrm{m}} / S_{\text {Radio }}\right)$, where $S_{\text {Radio }}$ is the radio flux density ${ }^{5}$. BL Lac candidates are located in the region of the two dimensional $w 1-w 2$ versus $w 2-w 3$ WISE color-color plane occupied by BL Lacs listed in the 5th edition of Roma-BZCAT and associated with $\gamma$-ray sources listed in the 3FGL. This region is bounded by the isodensity contour, obtained with kernel density estimation (KDE), containing 90\% of Roma-BZCAT $\gamma$-ray BL Lacs. Finally, the KDEBLLACS catalog is restricted to the sources outside the Galactic plane $\left(|b|>10^{\circ}\right)$. By cross-matching the $\mathrm{ABC}$ catalog with KDEBLLACS adopting a search radius of 3 '”0, we find only one match, namely the source J0835-5953, which is classified in the $\mathrm{ABC}$ catalog as a blazar.

\section{Multiwavelength analysis}

In this section, we aim to offer an improved characterization of our ABC sources by looking for their multiwavelength counterparts in the available catalogs obtained from major surveys.

\subsection{Optical data}

Optical observations of blazars both in photometry (e.g., Marchesini et al. 2016a; Raiteri et al. 2019; Abeysekara et al. 2020) and spectroscopy (e.g., Landoni et al. 2015; Ricci et al. 2015; Álvarez Crespo et al. 2016; Peña-Herazo et al. 2019; de Menezes et al. 2020) provide an excellent tool to characterize their broad band emission and a to pinpoint their classification through the detection (or lack of it) of strong emission lines. In this section, we collect the photometric and spectroscopic data available in public catalogs with the goal of obtaining a better characterization of the $\mathrm{ABC}$ sources.

\subsubsection{Galactic extinction}

As we saw in Sect. 2, nearly one-fourth of the ABC sources are located close to the Galactic plane, where extinction is a major issue. We used the Galactic Dust Reddening and Extinction

\footnotetext{
4 The thresholds on radio loudness are selected as $q_{22} \leq-0.61$, $q_{22} \leq-0.97$ and $q_{22} \leq-0.79$ for BZB, BZQ and MIXED candidates, respectively.

5 In particular, BL Lac candidates are selected with $-1.85<q_{12}<-1$, $-1.64<q_{12}<-1.01$ and $-1.64<q_{12}<-1$ for sources with NVSS, FIRST and SUMSS counterparts, respectively.
}

tool of the NASA/IPAC Infrared Science Archive ${ }^{6}$ to obtain the value of Galactic absorption along the line of sight of our $A B C$ sources, according to the analysis by Schlafly \& Finkbeiner (2011).

In Fig. 6, we show the distribution of the Galactic extinction in the $V$ band $A(V)$ (left panel) and the distribution of $A(V)$ as a function of the Galactic latitude (right panel) for both the ABC and Roma-BZCAT sources. We see that Roma-BZCAT objects have, on average, lower $A(V)$ values than $\mathrm{ABC}$ ones, confirming that an important percentage of $\mathrm{ABC}$ sources are probable blazars that have so far gone unrecognized because they lie close to the Galactic plane, where strong absorption makes optical observations difficult.

\subsubsection{Gaia}

The Gaia satellite (Gaia Collaboration 2016a) was launched in 2013 with the aim of performing the largest, most precise 3D map of our Galaxy. A first data release (DR1) was issued in 2016, covering the first 14 months of observations (Gaia Collaboration 2016b). A second data release (DR2) followed in 2018, including 22 months of observations (Gaia Collaboration 2018). Gaia DR2 contains high-precision parallaxes and proper motions for over 1 billion sources together with precise multiband photometry.

As stressed by Bailer-Jones et al. (2019), Gaia selects pointlike sources, so most galaxies have been missed. Moreover, parallaxes and proper motions of galaxies are liable to incorrect fitting by the astrometric model. Therefore, most BL Lac sources with dominant host galaxies (i.e., BZGs) may have no Gaia counterparts or may suffer for overestimates of parallaxes and proper motions.

We looked for Gaia counterparts of the ABC sources in DR2 using the standard 3".0 search radius and found 1137 matches. However, we expect that the Gaia counterparts can be shifted by no more than a fraction of arcsec with respect to the ALMA position. Therefore, in Fig. 7 we plot the distribution of the separations between the ABC source positions and its closest Gaia match. The separation distribution was fitted with Expectation-Maximization algorithm for mixtures of univariate normals (NORMALMIXEM ${ }^{7} \mathrm{R}$ package). We can clearly see that this distribution is adequately represented by two gaussians, the smallest one with mean $\mu_{1}=\sim 1^{\prime \prime}$.5 and the largest one with mean $\mu_{2}=\sim 2$ mas, indicating that the majority of these matches are accurate to the sub-arcsecond scale. We therefore select as reliable only the matches that show a separation smaller than $\sim 0{ }^{\prime \prime} 1$, that corresponds to a deviation of 3 sigmas from $\mu_{2}$, narrowing down the number of associations to 1030 .

In addition, extragalactic objects should ideally have null parallaxes and proper motions (even though not all sources with null parallax and proper motion are necessarily extragalactic objects). We then consider as possible identifications all associations where the proper motion and parallaxes are compatible with zero at a three-sigma level, narrowing down the number of associations to 805 possible identifications. These are shown in the left panel of Fig. 8 in the RA Dec proper motion plane, where we see that there is no significant difference between the proper motions of all possible identifications and sources close to the Galactic plane $\left(|b|<10^{\circ}\right)$.

In the right panel of the same figure we show Gaia parallaxes of the possible associations versus the separation between

\footnotetext{
6 https://irsa.ipac.caltech.edu/applications/DUST/

https://www.rdocumentation.org/packages/mixtools/ versions/1.0.4
} 

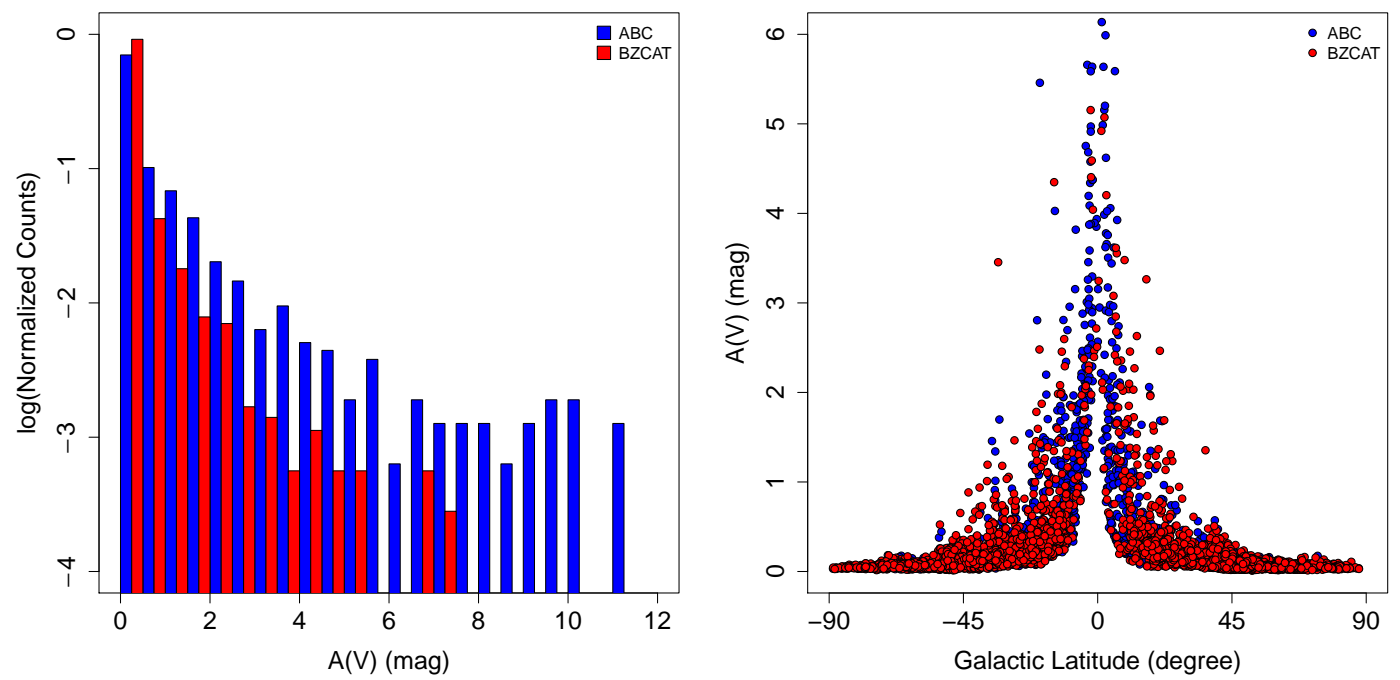

Fig. 6. Left panel: distribution of the Galactic extinction in the $V$ band $A(V)$ at the coordinates of $\mathrm{ABC}$ sources (blue bars) and at the coordinates of Roma-BZCAT sources (red bars). Right panel: galactic extinction in the $V$ band $A(V)$ versus Galactic latitude for ABC sources (blue circles) and for Roma-BZCAT sources (red circles).

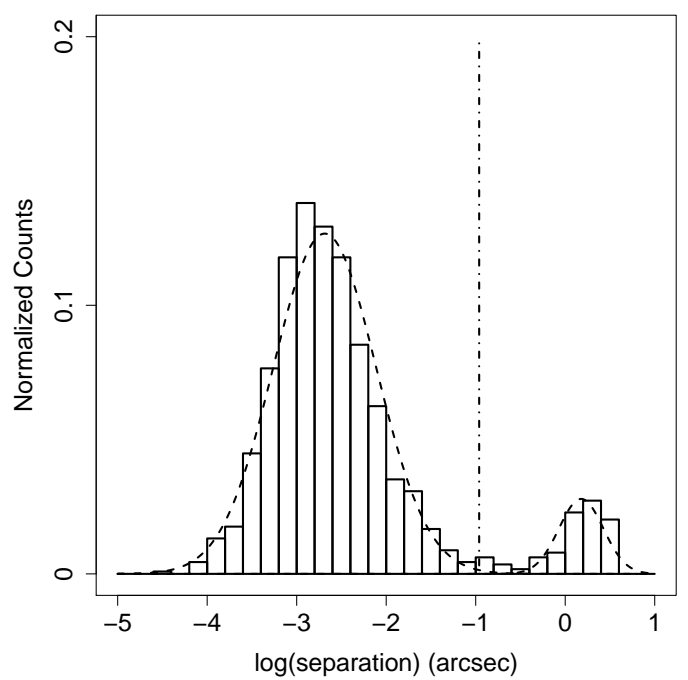

Fig. 7. Distribution of the separations between the ABC source positions and its closest Gaia match. The dashed lines represent the two gaussians fitting the separation distribution. The vertical dot-dashed line represents the three-sigma deviation from the mean of the largest gaussian, chosen as the boundary for reliable matches (see Sect. 4.1.2).

the ABC sources and their Gaia matches. We note that negative parallaxes are a consequence of how Gaia data are treated and can be safely used (Gaia Collaboration 2018). Again, we do not see a significant difference between the parallaxes of all possible identifications and sources close to the Galactic plane.

In Fig. 9, we compare the distribution of $\mathrm{ABC}$ sources (blue circles) and Roma-BZCAT sources (red circles) in the Gaia $g$ versus $b-r$ color-magnitude plot. The great majority $(\sim 98 \%)$ of the Gaia counterparts selected for ABC sources are detected in $g, b$, and $r$ bands. Gaia counterparts for RomaBZCAT sources have been selected adopting the same matching criteria used for ABC sources. All magnitudes have been corrected for Galactic absorption using reddening estimates from Schlafly \& Finkbeiner (2011) and the extinction model from Fitzpatrick \& Massa (2007). In the same plot we show with gray points in the background $\sim 5 \times 10^{4}$ random Gaia sources, together with KDE isodensity curves containing 60\%, 70\%, 80\%, and $90 \%$ of these Gaia random sources. On top and on the right of the main panel of this figure, we show the normalized distributions of the $b-r$ color and $g$ magnitude, respectively, for the ABC, Roma-BZCAT and random sources. It is evident that neither ABC nor Roma-BZCAT sources are clearly separated on the color-magnitude diagram from the random Gaia sources. $\mathrm{ABC}$ sources are, on average, slightly bluer and dimmer than the Roma-BZCAT ones, although spanning a similar range of magnitudes. A Kolmogorov-Smirnov (KS; Kolmogorov 1933; Smirnov 1939) test shows that the distributions of the Gaia $g$ magnitudes of ABC sources and Roma-BZCAT sources have a $p$-chance $p=3.0 \times 10^{-11}$ of having been randomly sampled from a common parent distribution, while for the $b-r$ color distributions this value is $p=1.1 \times 10^{-5}$.

We can then conclude that $\mathrm{ABC}$ sources are significantly dimmer than Roma-BZCAT sources in Gaia $g$ band, while the difference in the Gaia $b-r$ color between the two populations is less pronounced, while still significant.

\subsubsection{SDSS DR12 and LAMOST DR5}

The 12th data release of the Sloan Digital Sky Survey (SDSS DR12, Alam et al. 2015) covers over $14000 \mathrm{deg}^{2}$ of the sky, providing optical multiband photometric information for more than 450 millions unique objects and optical spectra for more than 5 million sources.

To determine the optimal cross-match radius of $\mathrm{ABC}$ sources with the SDSS DR12 catalog, we considered values from $00^{\prime \prime} 1$ to $33^{\prime \prime 0}$ in steps of $0 \prime \prime 1$. We then repeated the same procedure around random positions in the sky. In the left panel of Fig. 10, we compare the increase of sources with at least one SDSS counterpart $(\Delta n)$ with increasing search radius for the $\mathrm{ABC}$ sources (black line) and for the random positions (red line). Since these variations are rather noisy, for better visualization we smoothed $\Delta n$ using the SMOOTH.SPLINE $\mathrm{R}$ tool with a smoothing parameter 0.4 . For ABC sources, the majority of matches can already be found with a search radius of $00^{\prime \prime} 6$, and further increases in the search radius only add few matches, while for the random positions $\Delta n$ is initially 0 , and then it increases after $1^{\prime \prime} 0$. We therefore chose, as an optimal search radius for the SDSS DR12 

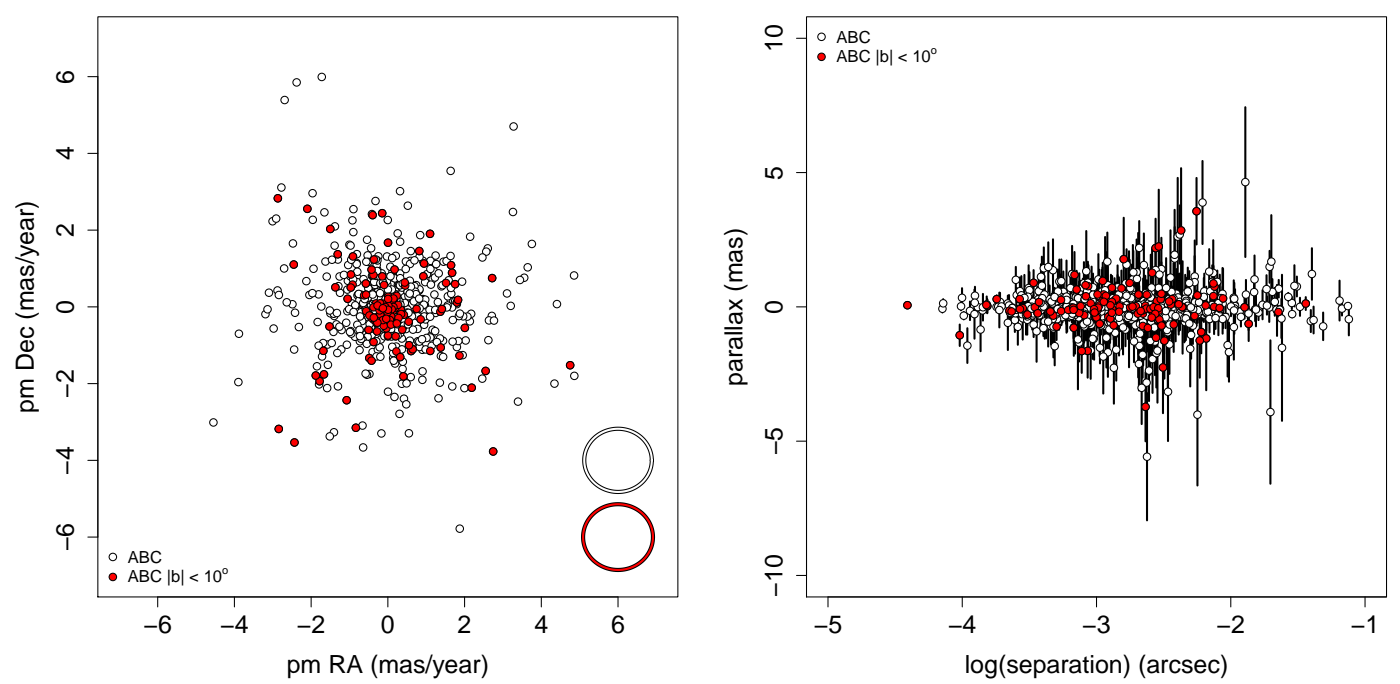

Fig. 8. Left panel: distribution of the 805 possible Gaia identifications in the motion plane (see Sect. 4.1.2). The white circles represent all possible Gaia identifications, and the red circles represent the sources close to the Galactic plane $\left(|b|<10^{\circ}\right)$. The ellipses in the lower-right corner represent the average uncertainties for possible Gaia identifications (white ellipse) and for the sources close to the Galactic plane (red ellipse). Right panel: Gaia parallaxes of the possible associations versus separation between the ABC sources and their Gaia matches. The vertical bars represent the 1- $\sigma$ errors on parallax, the white circles represent all possible Gaia identifications, and the red circles represent the sources close to the Galactic plane.

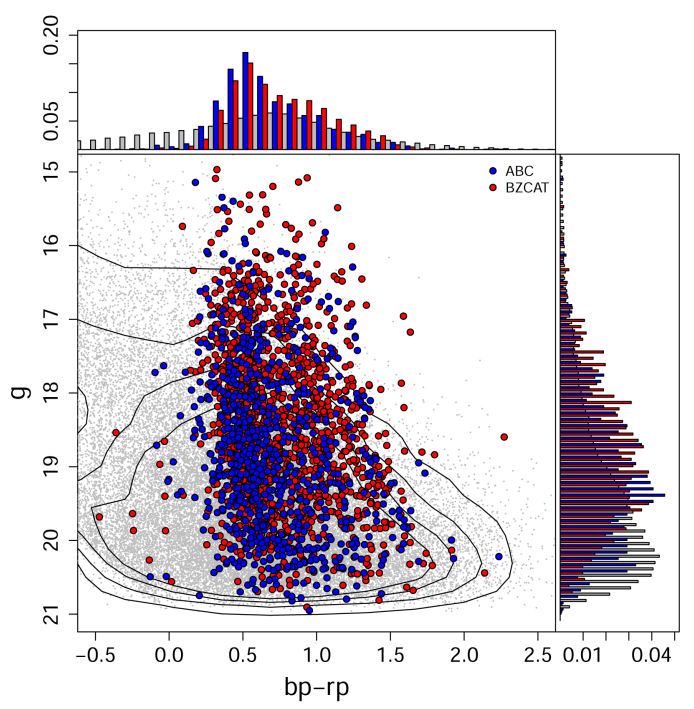

Fig. 9. Comparison of ABC sources (blue circles) and Roma-BZCAT sources (red circles) in the Gaia $g$ versus $b-r$ color-magnitude plot Gray points in the background represent random Gaia sources, and the black continuous lines indicate KDE isodensity curves containing $60 \%$, $70 \%, 80 \%$, and $90 \%$ of the Gaia random sources. On top and on the right of the main panel: we present the normalized distributions of the $b-r$ color and $g$ magnitude, respectively, for the ABC, Roma-BZCAT and random sources.

counterpart, the radius of 1 ". 1 at which the increase of matches at random positions in the sky becomes larger than that at the positions of ABC sources. As shown in the right panel of Fig. 10, at this radius we have a total of 295 SDSS DR12 matches for the ABC sources, without multiple matches. All these 295 sources are detected in the five SDSS bands.

The Large sky Area Multi-Object fiber Spectroscopic Telescope (LAMOST, Cui et al. 2012) survey is a large-scale spectroscopic survey which follows completely different target selection algorithms than SDSS, and is therefore complementary to the latter. The fifth data release of the LAMOST survey (DR5) provides optical spectra for more than 9 million sources.

We adopted the same approach to determine the optimal radius to cross-match $\mathrm{ABC}$ sources with the LAMOST DR5 catalog. We looked for matches in the latter with search radii, increasing from $00^{\prime \prime} 1$ to $33^{\prime \prime} 0$, with increments of $00^{\prime \prime} 1$, around both the coordinates of $\mathrm{ABC}$ sources and random positions in the sky. We find that for $\mathrm{ABC}$ sources, the majority of matches is already found with a search radius of $0{ }^{\prime \prime} .3$. Due to the difference in surface density of sources between SDSS DR12 ( 470 million sources) and LAMOST DR5 ( $\sim 7$ million sources), we start finding some matches around random sources only around 4". We then choose as an optimal search radius for LAMOST DR5 counterpart the radius of $0 \prime \prime 5$ at which $\Delta n$ reaches 0 , and above which fluctuations in $\Delta n$ for $\mathrm{ABC}$ sources and random positions are similar. At this radius, we have a total of 31 LAMOST DR5 matches for the ABC sources, without multiple matches. Of these, 29 have also SDSS DR12 counterparts.

In Fig. 11, we compare $\mathrm{ABC}$ sources (blue circles) and Roma-BZCAT sources (red circles) in the SDSS color-color plots, namely $g-r$ versus $u-g$ (left panel), $r-i$ versus $g-r$ (central panel), and $i-z$ versus $r-i$ (right panel). SDSS counterparts for Roma-BZCAT sources have been selected adopting the same matching radius used for ABC sources. Again, all magnitudes have been corrected for Galactic absorption. Like for Fig. 9, in the panels of Fig. 11 we show with gray points in the background $\sim 5 \times 10^{4}$ random SDSS sources together with KDE isodensity curves containing $60 \%, 70 \%, 80 \%$, and $90 \%$ of these random sources. Again, on the top and on the right of the main panels of this figure we show the normalized distributions of the SDSS color for the ABC, Roma-BZCAT and random sources. The ellipses in the panels indicate the average uncertainties on the SDSS colors.

We see that neither ABC nor Roma-BZCAT sources can be easily separated from random SDSS sources, with the possible exception of $g-r$ color, in which ABC and Roma-BZCAT sources appear bluer than the majority of random sources, however there is significant contamination. In general, ABC sources 

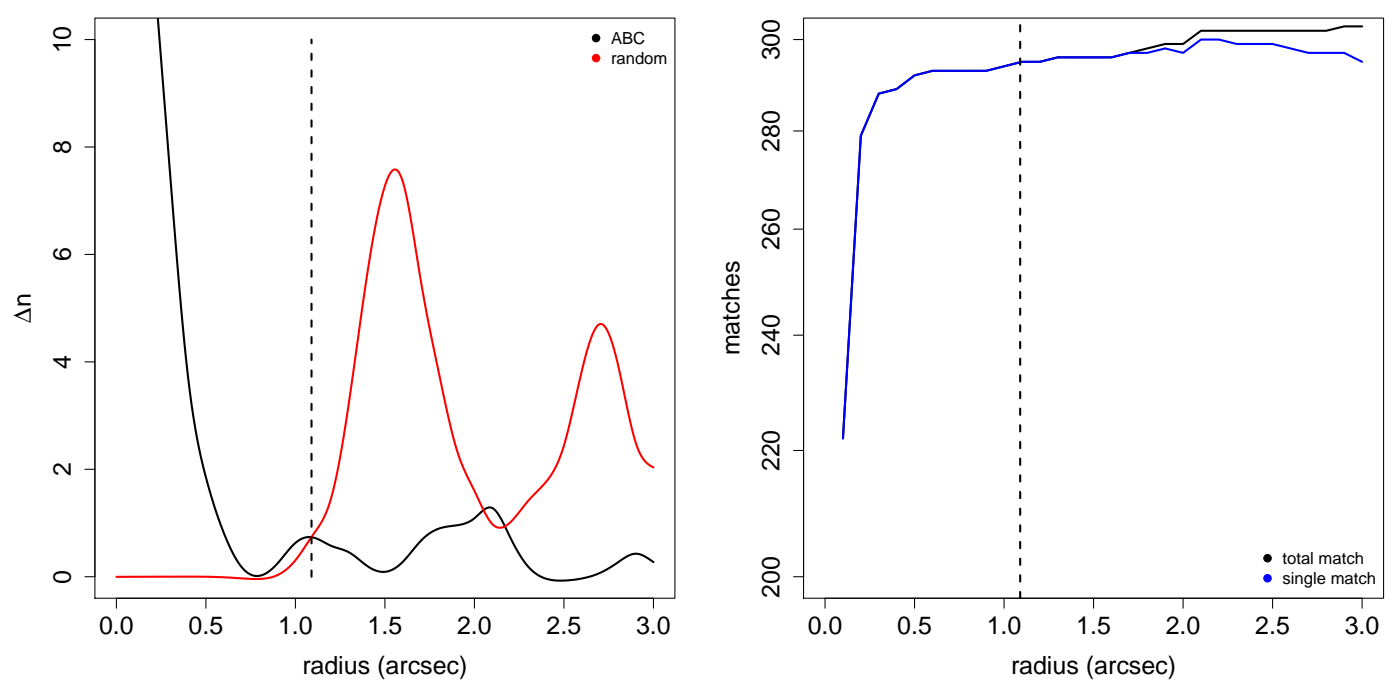

Fig. 10. Left panel: increase of sources with at least one SDSS counterpart $(\Delta n)$ plotted versus the search radius for ABC sources (black line) and for random positions in the sky (red line). Lines representing $(\Delta n)$ were smoothed for better visualization. The optimal search radius is represented with a vertical dashed line. Right panel: number of matches between ABC sources and SDSS DR12 sources plotted versus the search radius. The number of ABC sources with at least one match in SDSS DR12 is indicated with a black line, while the number of ABC the sources with a single match in SDSS DR12 is indicated with a blue line. As in the right panel, the optimal search radius is represented with a vertical dashed line.
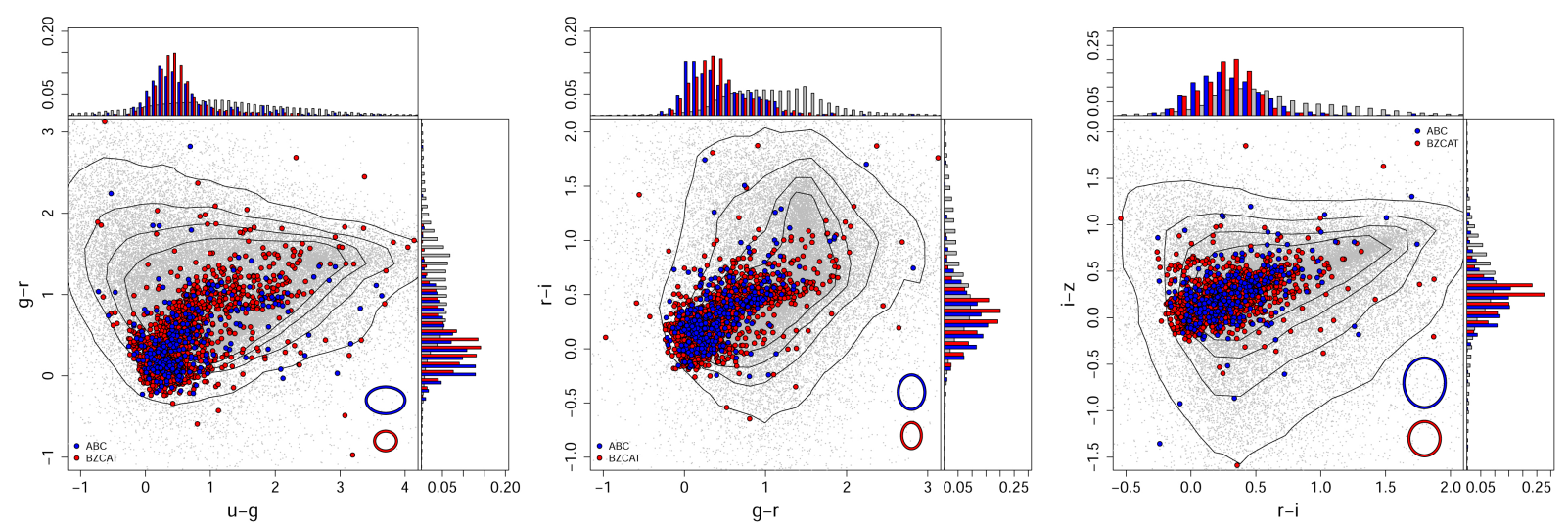

Fig. 11. Comparison of ABC sources (blue circles) and Roma-BZCAT sources (red circles) in the SDSS $g-r$ versus $u-g$ (left panel), $r-i$ versus $g-r$ (central panel), and $i-z$ versus $r-i$ (right panel) color-color diagrams. Gray points in the background represent random SDSS sources and the black continuous lines indicate $\mathrm{KDE}$ isodensity curves containing $60 \%, 70 \%, 80 \%$, and $90 \%$ of the random sources. On the top and on the right of each main panel: we show the normalized distributions of the SDSS colors for the ABC, Roma-BZCAT, and random sources. The ellipses in the panels indicate the average uncertainties on the SDSS colors.

appear bluer than Roma-BZCAT sources, however, the KS test for the distributions of the SDSS colors of ABC sources and Roma-BZCAT sources have $p$-chances of $p=5.7 \times 10^{-3}$, $p=5.5 \times 10^{-3}, p=4.4 \times 10^{-3}$ and $p=9.2 \times 10^{-5}$ for the $u-g, g-r, r-i$, and $i-z$ colors, respectively. Therefore, we can conclude that although ABC sources are on average bluer than Roma-BZCAT sources, these differences are not statistically significant.

In Fig. 12, we show the Roma-BZCAT (left panel) and ABC (right panel) sources on the SDSS $g-r$ versus $u-g$ color-color plane, superimposing the three regions defined by Butler \& Bloom (2011) to select low $(z<2.5)$, intermediate $(2.5<z<3)$, and high $(z>3)$ redshift quasars. For RomaBZCAT sources, we see that the majority of BZBs and BZQs fall into the region of low redshift quasars, with some BZQs falling into the regions of higher redshift quasars.

The BZGs, on the other hand, represent the majority of the Roma-BZCAT sources falling outside the quasar regions from Butler \& Bloom (2011), with some of the $u-g$ redder BZGs turning into the region of $z>3$ quasars. For ABC sources we have a similar situation, with most QSOs and BL Lacs falling into the region of low redshift quasars, and some QSOs entering the regions of higher redshift quasars. Sources outside the quasar regions from Butler \& Bloom (2011) are a mixed bag without a clear predominant class.

We then looked for available SDSS DR12 and LAMOST DR5 spectra for the previously identified counterparts, selecting only spectra without analysis flags. In SDSS DR12 we found 98 spectra, and in LAMOST DR5 we found 28 spectra (16 of which were already found in SDSS), for a total of 110 sources with available optical spectra; they are presented in Figs. B.1 and B.2. These spectra are classified either as QSO (98) or GALAXY (12) spectra, and the distribution of these spectral classes in ABC sources types is presented in Fig. 13. The majority of spectral classes in all ABC sources types is of course represented by QSOs, because these represent more than $90 \%$ of the sources with optical spectral classification. We can see, however, that one of the two $\mathrm{ABC}$ sources classified as blazars and as AGN have 

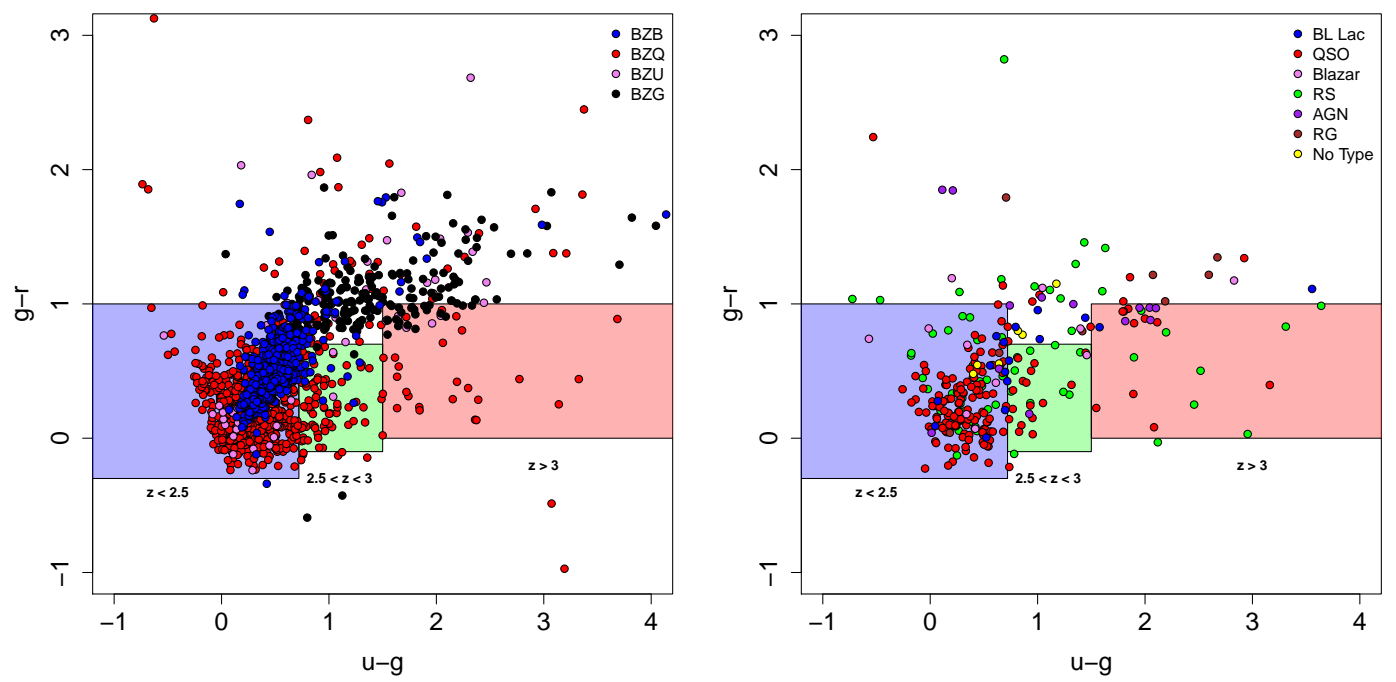

Fig. 12. Distribution of Roma-BZCAT (left panel) and ABC (right panel) sources in the SDSS $g-r$ versus $u-g$ color-color plane. The three colored rectangles represent the regions indicated by Butler \& Bloom (2011) to select low $(z<2.5)$, intermediate $(2.5<z<3)$, and high $(z>3)$ redshift quasars. Roma-BZCAT and ABC subclasses are represented with circles of different colors, as indicated in the legend.

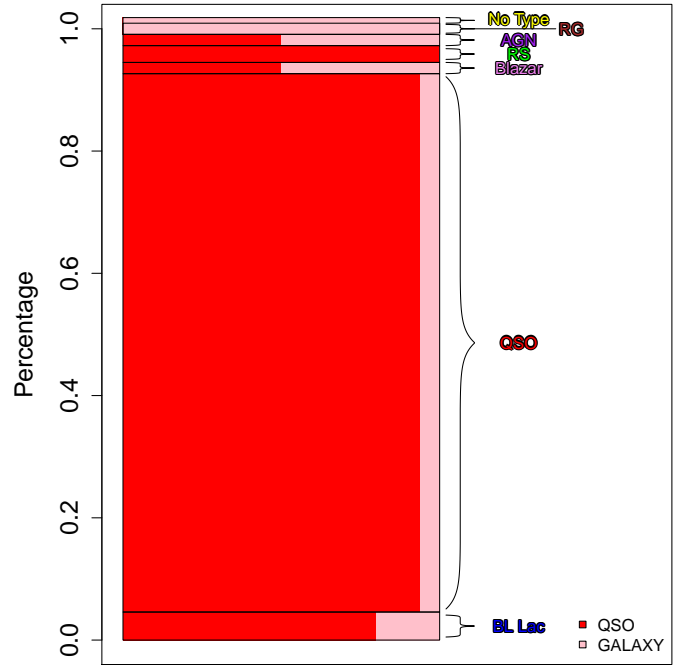

Fig. 13. Distribution of the spectral classes (QSO or GALAXY) of the 110 sources with an optical spectra in SDSS DR12 or LAMOST DR5 in the $\mathrm{ABC}$ sources types.

a GALAXY optical classification, just as the two ABC sources classified as RG and the one without literature classification. The optical properties of ABC sources are summarized in Table C.2, including the LAMOST and SDSS counterpart, spectral classification, and redshift.

\subsection{Infrared data: WISE}

WISE was launched in 2009, completing its double survey of the whole sky in 2011. The data were acquired with a $40 \mathrm{~cm}$ telescope in four filters, $w 1, w 2, w 3$, and $w 4$, with the central wavelength at $3.4,4.6,12$, and $22 \mu \mathrm{m}$, respectively. As mentioned previously, WISE data have been extensively used to select blazar candidates and study their properties (see also Massaro et al. 2012, 2015c; Plotkin et al. 2012; Capetti \& Raiteri 2015; Raiteri \& Capetti 2016). Released in 2013, the AllWISE catalog (Cutri et al. 2013) offers a compilation of the data from the cryogenic and post-cryogenic survey phases of the WISE mission, containing more than 700 million celestial objects.

The optimal search radius for cross-matching ABC and AllWISE catalogs was determined using the same procedure as for SDSS. In the left panel of Fig. 14, we compare the increase of sources with at least one AllWISE counterpart $(\Delta n)$ with an increasing search radius for the ABC sources (black line) and for the random positions (red line). Again, $\Delta n$ was smoothed for improved visualization. For ABC sources, the majority of matches was already found with a search radius of 1.15 , while for the random positions, $\Delta n$ is initially 0 and then increases after $11^{\prime \prime} 0$. We therefore chose as an optimal search radius for the WISE counterpart, a radius of 2 ". 4 , at which the increase of matches at random positions in the sky becomes larger than that at the positions of ABC sources. As shown in the right panel of Fig. 14, at this radius, we have a total of 1311 AllWISE matches for the ABC sources, without any multiple matches.

Specifically, we have 906 source with a detection in all four WISE bands, 739 of which $(\sim 82 \%)$ have a detection with a signal to noise ratio of at least 3 in all four WISE bands. The distribution of the $906 \mathrm{ABC}$ sources with a detection in all four WISE bands in the WISE color-color diagrams is presented with blue circles in Fig. 15, where the distribution of the Roma-BZCAT sources (red circles) is also shown for comparison. WISE counterparts for Roma-BZCAT sources were selected adopting the same matching radius used for $\mathrm{ABC}$ sources. The $w 1-w 2$ versus $w 2-w 3$ and $w 2-w 3$ versus $w 3-w 4$ color-color diagrams are presented in the left and right panel of the figure, respectively. The ellipses in the panels indicate the average uncertainties on the WISE colors. As usual, all magnitudes have been corrected for Galactic absorption. We note, however, that the average Galactic absorption is smaller than the average photometric uncertainties for $|b|>35^{\circ}$ and $|b|>20^{\circ}$ for $w 1$ and $w 2$ bands, respectively; and in these two bands, it becomes lager than 0.1 mag for $|b|<20^{\circ}$. On the other hand, Galactic absorption becomes relevant in $w 3$ and $w 4$ bands only very close to the Galactic center $\left(|b|<4^{\circ}\right.$, see also D'Abrusco et al. 2012). Again, on the top and on the right of the main panels we show the normalized distributions of the WISE color for the ABC and Roma-BZCAT sources. Sources from $\mathrm{ABC}$ have similar $w 1-w 2$ colors to Roma-BZCAT sources, while they appear bluer in the $w 2-w 3$ and $w 3-w 4$ colors. This 

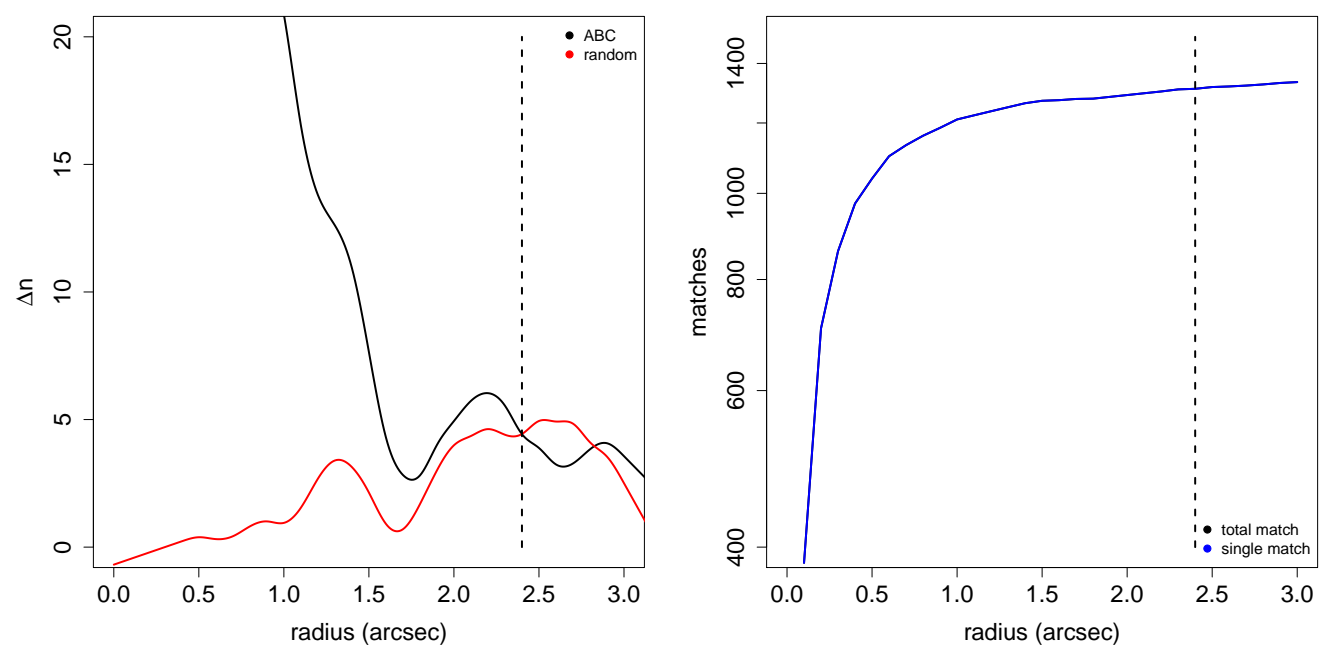

Fig. 14. Same as Fig. 10, but for matches between ABC sources and AllWISE source.
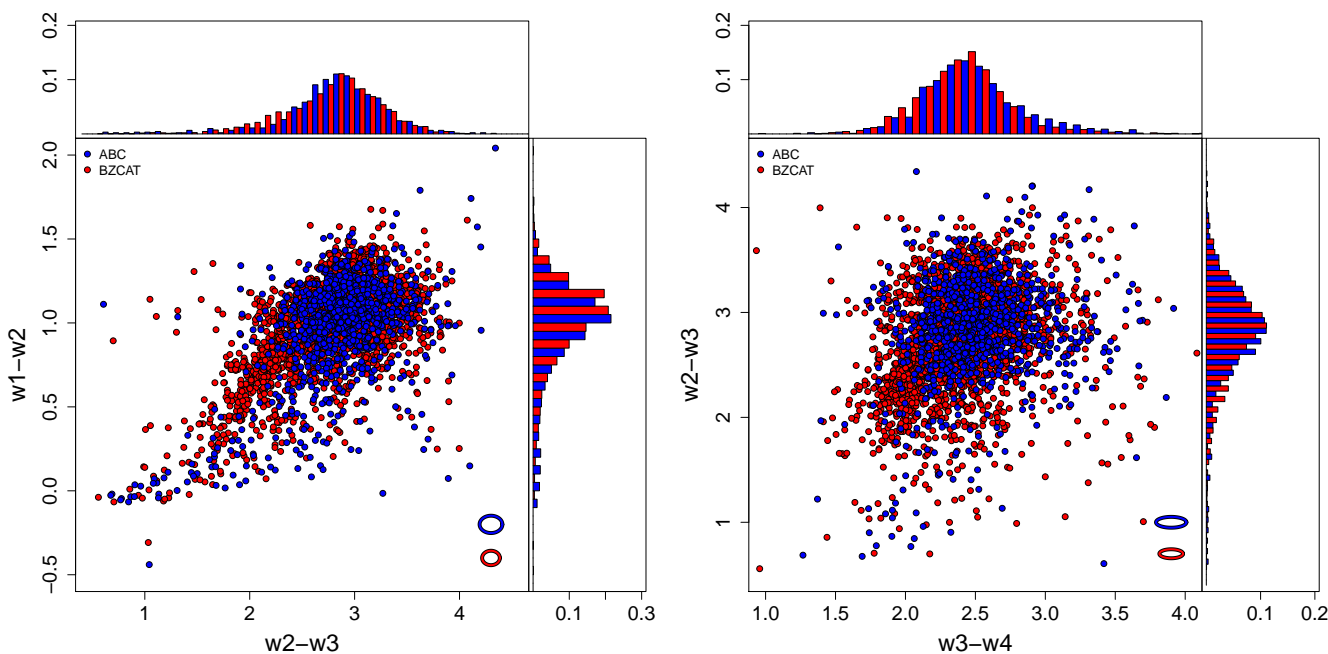

Fig. 15. Distribution of ABC sources (blue circles) and Roma-BZCAT sources (red circles) in the WISE $w 1-w 2$ versus $w 2-w 3$ (left panel) and $w 2-w 3$ versus $w 3-w 4$ (right panel) color-color diagrams. On the top and on the right of each main panel: we show the normalized distributions of the WISE colors for the ABC and Roma-BZCAT sources. The ellipses in the panels indicate the average uncertainties on the WISE colors.

is confirmed by the KS test for the distributions of the WISE colors of ABC sources and Roma-BZCAT sources that yields $p$ chances of $p=0.04, p=4.0 \times 10^{-4}$ and $p=8.9 \times 10^{-11}$ for the $w 1-w 2, w 2-w 3$, and $w 3-w 4$ colors, respectively. Therefore, we can conclude that the difference is significant only in the $w 2-w 3$ and $w 3-w 4$ colors.

\subsection{X-ray data}

Blazars are known X-ray sources since ROSAT DXRBS (Perlman et al. 1998; Landt et al. 2001) and Einstein IPC (Elvis et al. 1992; Perlman et al. 1999) surveys (see also Perlman 2000). Since then, the X-ray properties of blazars have been investigated in depth by numerous authors (see, e.g., example Giommi \& Padovani 1994; Padovani \& Giommi 1995; Comastri et al. 1997; Rieger et al. 2000; Donato et al. 2001; Massaro et al. 2011a,b).

The nature of X-ray emission in blazar is essentially nonthermal and the physical processes responsible for this emission vary in the different blazar subclasses. For HSPs, the synchrotron peak frequency $v_{\mathrm{p}}$ is larger than $10^{15} \mathrm{~Hz}$ and, therefore. the X-ray emission in these sources is dominated by the synchrotron radiation from the relativistic electrons in the jets. As the synchrotron peak frequency moves to lower energies the SSC component enters the X-ray band, and for low-synchrotron peaked blazars (LSPs) with $v_{\mathrm{p}}<10^{14} \mathrm{~Hz}$, this is the dominant contribution to the observed X-ray emission. This is even more true in FSRQs, where the synchrotron peak frequency is $v_{\mathrm{p}}<10^{13} \mathrm{~Hz}$ and the $\mathrm{X}$-ray emission is dominated by the inverse Compton components (SSC and EC). To study the X-ray emission from the ABC sources, we made use of the data available for this sample in the archives of Swift, Chandra and XMM-Newton missions.

\subsubsection{Swift-XRT}

Swift has proven to be an excellent multifrequency observatory for blazar research, having so far observed hundreds of sources (e.g., Moretti et al. 2007, 2012; Dai et al. 2012) and providing an extremely rich and unique database of multifrequency (optical, UV, X-ray), simultaneous blazar observations. Several papers on samples selected with different criteria have already been published, including: blazars detected at $\mathrm{TeV}$ energies 
(e.g., Massaro et al. 2008, 2011a,b), simultaneous optical-to-Xray observations of flaring TeV sources (e.g., Perri et al. 2007; Tramacere et al. 2007), as well as the investigation of lowand high-frequency peaked BL Lacs (e.g., Maselli et al. 2010; Giommi et al. 2012). Swift has also been used for UV-optical and X-ray follow-up observations of $\mathrm{TeV}$ flaring blazars (e.g., Aliu et al. 2011; Aleksić et al. 2012; H.E.S.S. Collaboration 2013) and in the framework of broad-band multiwavelength campaigns (e.g. Raiteri et al. 2011, 2013; Carnerero et al. 2017). It has also been useful in obtaining photometric redshift constraints for many Fermi-detected BL Lacs (Rau et al. 2012).

Data collected by Swift X-Ray Telescope (XRT) were downloaded from the HEASARC ${ }^{8}$ data archive and processed using the XRTDAS software (Capalbi et al. 2005) developed at the ASI Science Data Center and included in the HEAsoft package (v. 6.26.1) distributed by HEASARC, using a procedure similar to the one illustrated in Paggi et al. (2013) and Marchesini et al. (2019). Swift-XRT photon counting (PC) data were available for 325 ABC sources. For each observation, calibrated and cleaned PC mode event files were produced with the XRTPIPELINE task (ver. 0.13.5), generating exposure maps for each observation. In addition to the screening criteria used by the standard pipeline processing, we applied a further filter to screen background spikes that can occur when the angle between the pointing direction of the satellite and the bright Earth limb is low. In order to eliminate this so-called bright Earth effect, due to the scattered optical light that usually occurs towards the beginning or the end of each orbit, we used the procedure proposed by Puccetti et al. (2011) and D'Elia et al. (2013). We monitored the count-rate on the CCD border and, through the XSELECT package, we excluded time intervals when the count-rate in this region exceeded 40 counts $\mathrm{s}^{-1}$. In addition, we selected only time intervals with $\mathrm{CCD}$ temperatures less than $-50^{\circ} \mathrm{C}$ (instead of the standard limit of $-47^{\circ} \mathrm{C}$ ) since contamination by dark current and hot pixels, both of which increase the low energy background, are strongly temperature dependent (D'Elia et al. 2013).

To detect X-ray sources in the XRT images, we made use of the XIMAGE detection algorithm DETECT, which locates the point sources using a sliding-cell method. The average background intensity is estimated in several small square boxes that are uniformly located within the image. The position and intensity of each detected source are calculated in a box whose size maximizes the signal-to-noise ratio. The algorithm was set to work in bright mode, which is recommended for crowded fields and fields containing bright sources since it can reconstruct the centroids of very nearby sources. We then evaluated the net count-rates for the detected sources with the SOSTA algorithm that, besides the net count-rates and the respective uncertainties, yields the statistical significance of each source. We note that SOSTA requires the positions of the sources detected by DETECT, and the uncertainties in the count-rates returned by SOSTA are purely statistical - meaning that do not include systematic errors - and they are, in general, smaller than those given by DETECT. We used count-rates produced by SOSTA because these are in most cases more accurate since DETECT uses a global background for the entire image, whereas SOsTA uses a local background. Finally, we refined the source position and relative positional errors by using the task XRTCENTROID of the XRTDAS package, considering only the sources detected at a position that is compatible with the ABC sources. In this way, we detected $\mathrm{X}$-Ray counterparts for 101 sources.

8 https://heasarc.gsfc.nasa.gov/
Generally, XRT-PC source spectra - with the corresponding arf and rmf files - were obtained from events extracted with XRTPRODUCTS task, using a 30 pixel radius circle centered on the detected source coordinates, while background spectra were estimated from a nearby source-free circular region of 60 pixel radius. When the source count-rate is above 0.5 counts $\mathrm{s}^{-1}$, the data are significantly affected by pileup in the inner part of the point spread function (PSF, Moretti et al. 2005). To remove the pile-up contamination, we extracted only those events contained in an annular region centered on the source (Perri et al. 2007). The inner radius of the region was determined by comparing the observed profiles with the analytical model derived by Moretti et al. (2005) and typically has a four or five pixel radius. In this way, we were able to obtain X-ray for spectra $43 \mathrm{ABC}$ sources.

\subsubsection{Chandra-ACIS}

Chandra X-ray telescope has been used in the past years to provide important information about the high-energy emission of blazars (e.g. Ighina et al. 2019). In addition, thanks to its unmatched angular resolution, Chandra has been used to resolve and study the X-ray jets of several blazars (e.g., Jorstad \& Marscher 2004; Tavecchio et al. 2007; Marscher \& Jorstad 2011; Hogan et al. 2011).

Data collected by the Chandra Advanced CCD Imaging Spectrometer (ACIS) were available for fields containing 62 ABC sources. These were retrieved from the Chandra Data Archive $^{9}$ and analyzed with CIAO (Fruscione et al. 2006) data analysis system version 4.12 and Chandra calibration database CALDB version 4.9.1, adopting standard procedures. We then ran the ACIS level 2 processing with CHANDRA_REPRO CIAO task to apply up-to-date calibrations (CTI correction, ACIS gain, bad pixels) and then excluded time intervals of background flares exceeding $3 \sigma$ with the DEFLARE task. We produced full-band exposure maps, PSF maps to evaluate the PSF size across the ACIS detector, and pileup maps with the PILEUP_MAP task. We then run the WAVDETECT task to identify point sources in each observation with a $\sqrt{2}$ sequence of wavelet scales (i.e., 11.41 22.8345 .66811 .3116 pixels) and a false-positive probability threshold of $10^{-6}$. We then considered only the sources detected at positions compatible with the ABC sources and extracted count-rates, making use of the SRCFLUX task. In this way, we detected X-ray counterparts for 56 ABC sources.

ACIS source spectra and the corresponding arf and rmf files were extracted with the SPECEXTRACT tool from the source regions generated from WAVDETECT, excluding the inner pixels with pileup larger than $5 \%$ as estimated from the pileup maps, while background spectra were extracted from source-free circular regions with typical radii of $80^{\prime \prime}$. In this way, we were able to extract spectra for $46 \mathrm{ABC}$ sources.

\subsubsection{XMM-Newton-EPIC}

The XMM-Newton space observatory, thanks to its large collecting area the ability to make long uninterrupted observations, has provided important information for the multiwavelength study of blazars (e.g., Raiteri et al. 2006; Fidelis et al. 2009; Kalita et al. 2015; Bhagwan et al. 2016).

Data collected by XMM-Newton European Photon Imaging Camera (EPIC), available for fields containing 64 ABC sources,

\footnotetext{
9 http://cda.harvard. edu/chaser
} 
Table 2. X-ray properties of ABC sources.

\begin{tabular}{lccccccc}
\hline \hline Name ALMA & Detector & $\begin{array}{c}\text { EXP } \\
\mathrm{ks}\end{array}$ & $\begin{array}{c}\text { Count rate } \\
10^{-3} \mathrm{~s}^{-1}\end{array}$ & $S / N$ & $\begin{array}{c}\operatorname{Flux}_{0.1-2.4} \\
10^{-13} \mathrm{erg} \mathrm{cm}^{2} \mathrm{~s}^{-1}\end{array}$ & $\Gamma_{\mathrm{X}}$ & $\begin{array}{c}K \\
10^{-4} \mathrm{keV} \mathrm{cm}^{-2} \mathrm{~s}^{-1}\end{array}$ \\
\hline J0010-0433 & XRT & 1.3 & & & & & \\
J0011-8706 & XRT & 4.9 & $2.55 \pm 0.84$ & 3.0 & $0.87 \pm 0.29$ & & \\
J0019-5641 & XRT & 2.9 & & & & & \\
J0024-6820 & XRT & 0.7 & $13.25 \pm 5.00$ & 2.6 & $3.82 \pm 1.44$ & & $0.53_{-0.10}^{+0.11}$ \\
J0025+3919 & ACIS & 19.7 & $15.70 \pm 1.00$ & 15.7 & $2.04 \pm 0.35$ & $1.36_{-0.22}^{+0.22}$ & \\
J0028+2000 & XRT & 3.0 & & & & & \\
J0029+3456 & EPIC & 28.2 & $66.77 \pm 3.08$ & 21.7 & $2.15 \pm 0.10$ & & \\
J0034-0054 & ACIS & 2.0 & $14.60 \pm 2.90$ & 5.0 & $2.54 \pm 0.50$ & & \\
J0039-2220 & XRT & 0.8 & $12.42 \pm 4.30$ & 2.9 & $3.51 \pm 1.21$ & & \\
J0045-3705 & XRT & 2.2 & & & & & \\
\hline
\end{tabular}

Notes. For each source we list the name (Name ALMA), the X-ray detector (Detector) and the observation exposure (EXP). If the source is detected we also list the count-rate (Count rate) and the signal to noise ratio $(S / N)$. We then list the unabsorbed source flux (Flux $x_{0.1-2.4}$ ) in the 0.1-2.4 KeV band, obtained from the count-rate assuming a power-law model with a spectral index 1.8 or, if a spectrum is available, from the spectral fit. For the sources adequately fitted with a power-law model, we also list the power-law spectral index $\Gamma_{\mathrm{X}}$ and normalization $K$, together with the fit $\chi^{2}$ and degrees of freedom ( $\chi^{2}$, d.o.f.). The full table is available in electronic form at the CDS.

were retrieved from the XMM-Newton Science Archive ${ }^{10}$ and reduced with the SAS ${ }^{11} 18.0 .0$ software.

Following Nevalainen et al. (2005) we filtered EPIC data for hard-band flares by excluding the time intervals where the 9.5-12 keV (for MOS1 and MOS2) or 10-12 keV (for PN) count-rate evaluated on the whole detector FOV was more than $3 \sigma$ away from its average value. To achieve a tighter filtering of background flares, we iteratively repeated this process two more times, re-evaluating the average hard-band count-rate and excluding time intervals away more than $3 \sigma$ from this value. The same procedure was applied to soft $1-5 \mathrm{keV}$ band restricting the analysis to an annulus with inner and outer radii of $12^{\prime}$ and $14^{\prime}$ (excluding sources in the field) where the detected emission is expected to be dominated by the background.

When possible, we merged data from MOS1, MOS2 and PN detectors from all observations using the MERGE task, in order to detect the fainter sources that wouldn't have been detected otherwise. Sources were detected on these merged images following the standard SAS sliding box task EDETECT_CHAIN that mainly consist of three steps: (1) source detection with local background, with a minimum detection likelihood of 8 ; (2) removal of sources found in step 1 and creation of smooth background maps by fitting a 2D spline to the residual image; (3) source detection with the background map produced in step 2 with a minimum detection likelihood of 10. The task EMLDETECT was then used to determine the parameters for each detected source including the count rate - by means of a maximum-likelihood fit to the input images, selecting sources with a minimum detection likelihood of 15 and a flux in the $0.3-7 \mathrm{keV}$ band larger than $10^{-14} \mathrm{erg} \mathrm{cm}^{-2} \mathrm{~s}^{-1}$ (assuming an energy conversion factor of $1.2 \times 10^{-11} \mathrm{cts} \mathrm{cm}^{2} \mathrm{erg}^{-1}$ ). An analytical model of the PSF was evaluated at the source position and normalized to the source brightness. The source extent was then evaluated as the radius at which the PSF level equals half of local background. We then considered only the sources detected at position compatible with the $\mathrm{ABC}$ sources. In this way, we detected X-ray counterparts for $55 \mathrm{ABC}$ sources.

The source spectra were extracted with the EVSELECT task from the regions obtained with EMLDETECT. The inner regions of high pileup were estimated using the EPATPLOT task through

\footnotetext{
${ }^{10}$ http://nxsa.esac.esa.int/nxsa-web

11 http://www . cosmos.esa.int/web/xmm-newton/sas
}

the distortion of pattern distribution, following the procedure explained in the SAS Data Analysis Threads ${ }^{12}$. The corresponding arf and rmf files were generated with the RMFGEN and ARFGEN tasks to take into account time and position-dependent EPIC responses, and background spectra were extracted from source free regions of the sky. We were able to extract spectra for 51 $\mathrm{ABC}$ sources.

\subsubsection{X-Ray Spectral Fitting}

In total, we detected X-ray counterparts for $173 \mathrm{ABC}$ sources and we were able to extract a total of 140 spectra for 92 ABC sources. Spectral fitting was performed with the Sherpa ${ }^{13}$ modeling and fitting application (Freeman et al. 2001) in the $0.3-7 \mathrm{KeV}$ energy range, adopting Gehrels weighting (Gehrels 1986). Source spectra were binned to a minimum of 20 counts/bin to ensure the validity of $\chi^{2}$ statistics. For the EPIC spectra we excluded from the spectral fitting the $1.45-1.55 \mathrm{KeV}$ band due to variable $\mathrm{Al} \mathrm{K}$ lines, and fitted simultaneously the MOS1, MOS2, and PN spectra.

For the spectral fitting, we used a model comprising an absorption component fixed to the Galactic value (Kalberla et al. 2005) and a power law, as expected in studies of blazars. This model proved to adequately fit the majority (109) of the extracted spectra. The results of the power-law model fitting are presented in Table 2, where errors correspond to the 1- $\sigma$ confidence level for one interesting parameter $\left(\Delta \chi^{2}=1\right)$. The X-ray spectra fitted with power-law models are presented in Figs. A.1-A.3. The intrinsic (i.e., unabsorbed) fluxes listed in Table 2 are estimated from the spectral fitting when a spectra was available, otherwise they are estimated from the measured count-rates assuming a power-law model with a spectral index of 1.8.

We note that 31 spectra for 24 ABC sources were not adequately fitted by a simple power-law model but they required, instead, more complex models comprising intrinsic absorption, thermal components, reflections components, or emission lines. They are presented in Figs. A.4-A.6. The results of the fit procedure on these spectra are summarized in Table 3. Interestingly, only one of these 24 sources showing complex X-ray spectra

\footnotetext{
12 https: //www . cosmos. esa. int/web/xmm-newton/sasthread-epatplot

13 http://cxc.harvard. edu/sherpa
} 


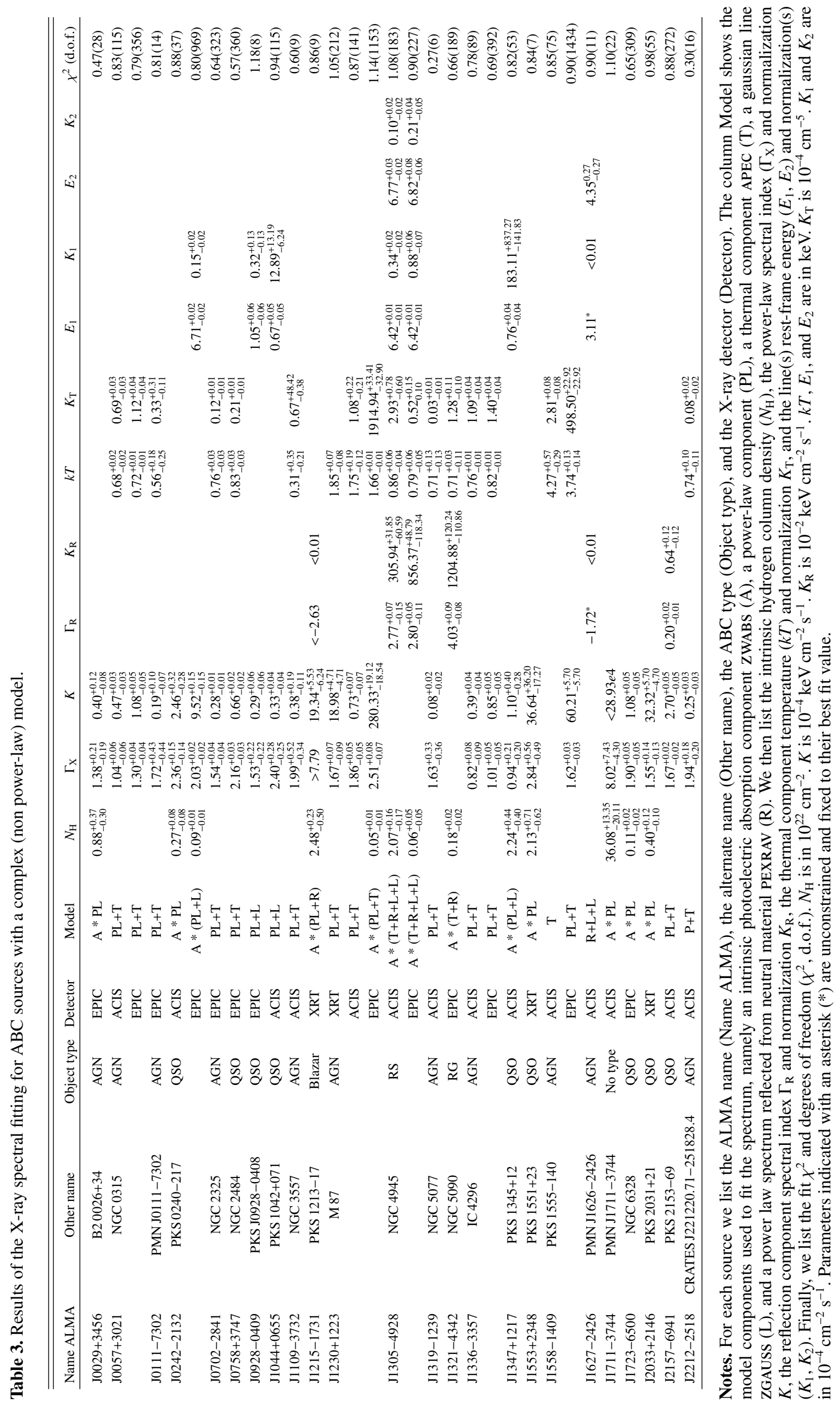



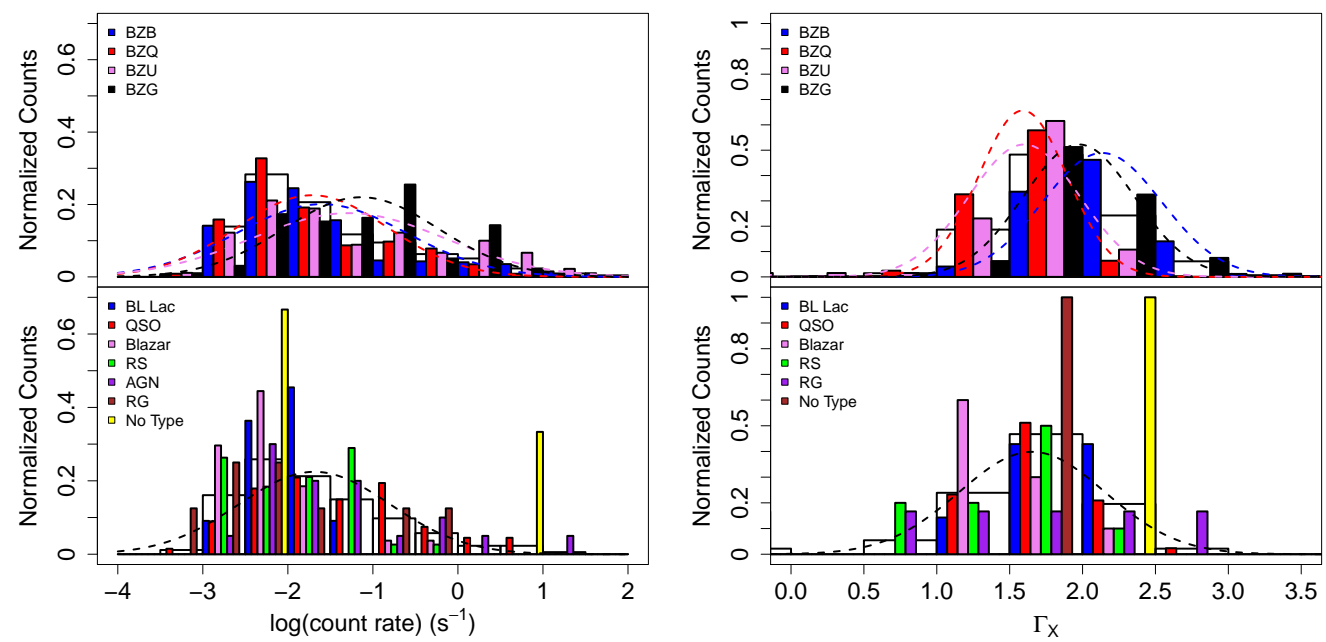

Fig. 16. Left panel: normalized distributions of the $0.3-7 \mathrm{KeV}$ count-rate for Roma-BZCAT (top) and ABC (bottom) sources. The various subclasses of Roma-BZCAT sources and types of ABC sources are presented with the colors indicated in the legend, and the white histograms on the background represent the distribution of the whole samples. For Roma-BZCAT sources, gaussian fits to the subclass distributions are indicated with dashed lines of the respective colors, while for $\mathrm{ABC}$ sources a gaussian fit to the distribution of the whole sample is indicated with a black dashed line. Right panel: same as the right panel, but for the distributions of the $\mathrm{X}$-ray slope $\Gamma_{\mathrm{X}}$.

(namely J1215-1731) has a blazar classification in SIMBAD, while the others are AGNs (11 sources), QSOs (9), RSs (1), RGs (1), and objects without SIMBAD classification (1).

It is useful to compare the $\mathrm{X}$-ray properties of $\mathrm{ABC}$ and Roma-BZCAT sources. To this end, we obtained and reduced Swift-XRT, Chandra-ACIS and XMM-Newton data for RomaBZCAT sources in the same way as we did for ABC sources. In the left panel of Fig. 16, we compare the normalized distributions of the X-ray count-rates for Roma-BZCAT (top) and ABC (bottom) sources. When count-rates for a source were available for more than one instrument, we picked the count-rate corresponding to the detection with the higher signal to noise ratio. The Roma-BZCAT sources of the different subclasses (BZB, $\mathrm{BZQ}, \mathrm{BZU}$, and BZG) are presented with different colors, and gaussian fits to the count-rate distributions of each subclass are indicated with dashed lines of the respective color. The peak logarithmic count-rates (in units of $\mathrm{s}^{-1}$ ) of BZBs and BZQs are at -1.6 and -1.7 , respectively, while BZUs and BZGs are slightly brighter on average, peaking at -1.3 and -1.1 , respectively. For ABC sources, the different source types are indicated with different colors presented in the legend. Due to the lower statistics with respect to the Roma-BZCAT we overplot only a gaussian fit to the distribution of the whole sample, presented with a dashed black line. ABC sources show similar count-rates in X-rays compared to Roma-BZCAT sources, at variance with what is observed at radio wavelengths (see Fig. 2). In particular, the distribution of logarithmic count-rates for Roma-BZCAT sources peaks at -1.6 , while that of ABC sources peaks at -1.7 . In addition, the KS test shows that these two distributions have a $p$-chance, $p=0.44$, of having been randomly sampled from a common parent distribution and are, therefore, statistically indistinguishable.

The right panel of Fig. 16 shows the comparison of the power-law slopes, $\Gamma_{X}$, of the X-ray spectra in terms of count rates. Again, when $\Gamma_{X}$ slopes for a source were available for more than one instrument, we picked the slope corresponding to the detection with the higher signal to noise ratio. BZBs and BZGs are the softer subclasses in X-rays, with their $\Gamma_{X}$ distributions peaking at 2.1 and 2.0, respectively, while BZQs and BZUs are harder, both peaking at 1.6. The ABC sources have a

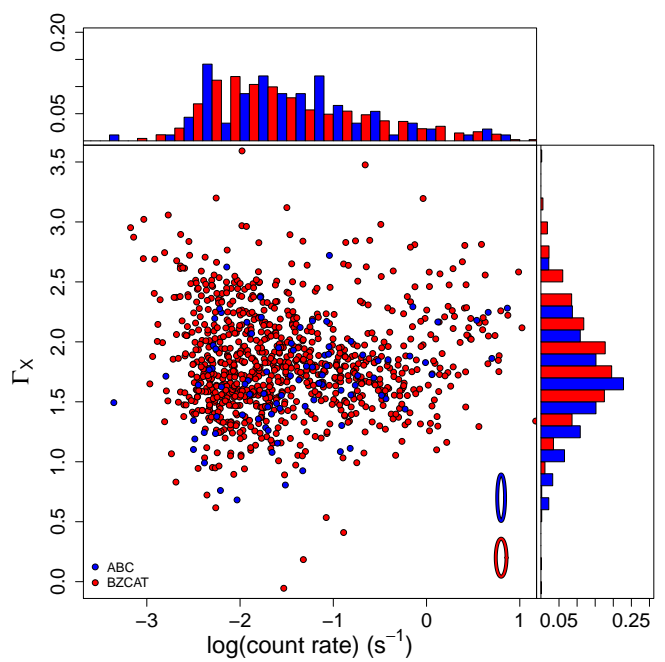

Fig. 17. ABC (blue circles) and Roma-BZCAT (red circles) sources represented on the X-ray slope versus X-ray count-rate plot. The ellipses of the relative color indicate the average uncertainties. On top and on the right of the main panels: we show the normalized distributions of the $\mathrm{X}$-ray count-rate and slope for ABC and Roma-BZCAT sources, with the same colors used in the main panel.

$\Gamma_{\mathrm{X}}$ distribution similar to that of the Roma-BZCAT as a whole. The ABC distribution peaks at $\Gamma_{X}=1.7$, while that of RomaBZCAT sources as whole peaks at 1.8. Also, the KS test shows that these two distributions have a $p$-chance, $p=0.10$, of having been randomly sampled from a common parent distribution, making them, therefore, similar.

In Fig. 17, we present on a X-ray slope versus X-ray countrate plot the $\mathrm{ABC}$ and Roma-BZCAT sources that have an estimate for both count-rate and X-ray slope. On the top and right of this figure, we present the normalized distributions of the X-ray count-rate and slope $\Gamma_{\mathrm{X}}$ for $\mathrm{ABC}$ and Roma-BZCAT sources. Both count-rate and $\Gamma_{X}$ distributions are similar among $A B C$ and Roma-BZCAT sources, and this is confirmed by the KS test, which yields a $p$-chance of 0.11 and 0.10 for the count-rate and $\Gamma_{\mathrm{X}}$ distributions, respectively. 

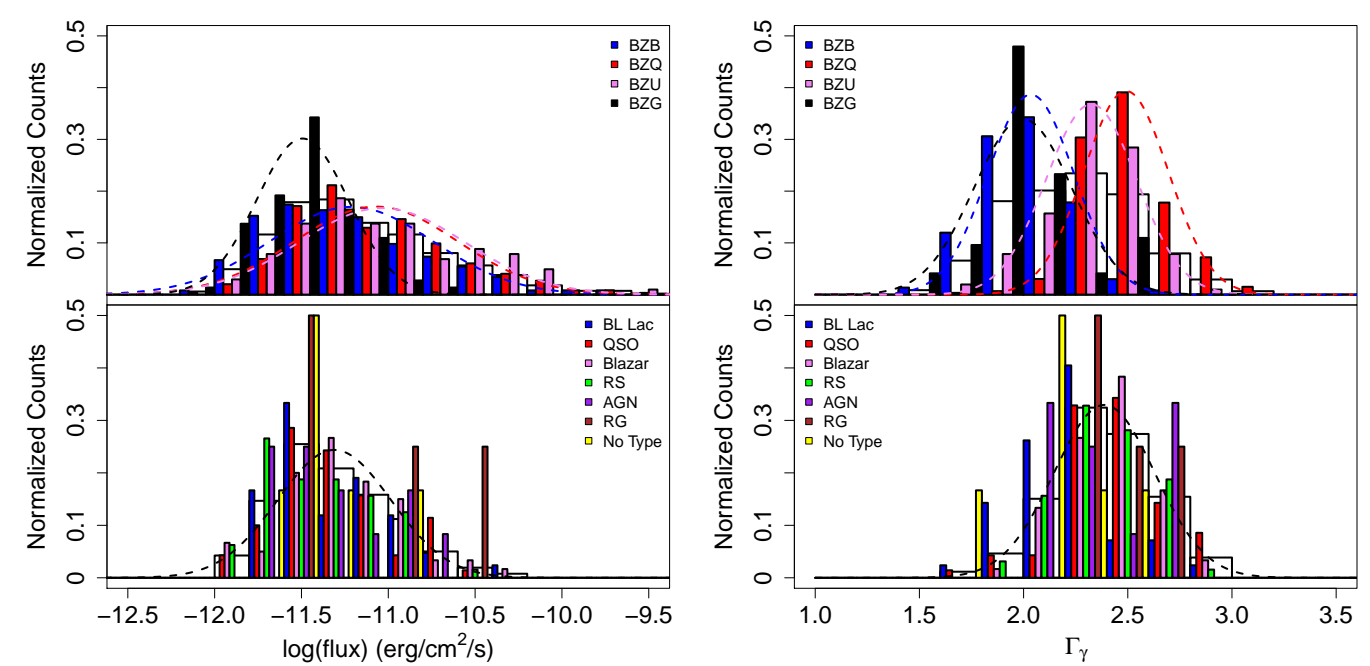

Fig. 18. Left panel: normalized distributions of the $100 \mathrm{MeV}-100 \mathrm{GeV}$ flux for Roma-BZCAT (top) and ABC (bottom) sources. The various subclasses of Roma-BZCAT sources and types of ABC sources are presented with the colors indicated in the legend, and the white histograms on the background represent the distribution of the whole samples. For Roma-BZCAT sources, gaussian fits to the subclass distributions are indicated with dashed lines of the respective colors, while for $\mathrm{ABC}$ source a gaussian fit to the distribution of the whole sample is indicated with a black dashed line. Right panel: same as the right panel, but for the distributions of the $\gamma$-ray slope $\Gamma_{\gamma}$.

\section{4. $\gamma$-ray data: Fermi-LAT}

In this section, we compare the $\gamma$-ray properties of $\mathrm{ABC}$ and Roma-BZCAT sources, as reported in 4FGL catalog. In Sect. 3, we explain our finding of 259 4FGL counterparts (mainly BCUs) for ABC sources. In addition, we find 1506 Roma-BZCAT sources with a counterpart in the 4FGL catalog. For each of these sources, we collected from the 4FGL the photon index obtained fitting the Fermi Large Area Telescope (LAT) spectra with a power-law (PL_INDEX PHOTON) and the energy flux in the range $100 \mathrm{MeV}-100 \mathrm{GeV}$ obtained from the spectral fitting (ENERGY_FLUX100), mostly ( 70\%) with a power-law model. Although these two quantities are not independent, it is instructive to compare their distributions in Roma-BZCAT and ABC sources.

As in Fig. 16, in the left panel of Fig. 18, we compare the normalized distributions of the $\gamma$-ray flux for Roma-BZCAT (top) and $\mathrm{ABC}$ (bottom) sources. The Roma-BZCAT of the different subclasses (BZB, BZQ, BZU, and BZG) are presented with different colors and gaussian fits to the flux distributions of each subclass are indicated with dashed lines of the respective color. The peak logarithmic fluxes (in cgs units) of these fits are similar for BZQs and BZUs, being, -11.1 and -11.0 , respectively, while BZGs are on average dimmer, peaking at -11.5 . BZBs sit somewhat in between, peaking at -11.2 . For $A B C$ sources the different source types are indicated with different colors. Again, due to lower statistics with respect to the Roma-BZCAT, we overplot just a gaussian fit to the distribution of the whole sample. We see that Roma-BZCAT sources extend to larger fluxes with respect to $\mathrm{ABC}$ sources, which is at variance with what is observed at radio wavelengths (see Fig. 2). We note that both distributions of $\gamma$-ray logarithmic flux peak at similar values, with the RomaBZCAT sources peaking at -11.3 , and the $\mathrm{ABC}$ sources peaking at -11.3 , between BZG and BZB peaks. However a KS test shows that the two distributions have a $p$-chance, $p=1.3 \times 10^{-5}$, of having been randomly sampled from a common parent distribution, making them, therefore, significantly different from the statistical point of view.

The right panel of Fig. 18 shows a similar comparison, but for the power-law slopes $\Gamma_{\gamma}$ of the Fermi-LAT spectra. RomaBZCAT subclasses are clearly separated on the basis of their spec-

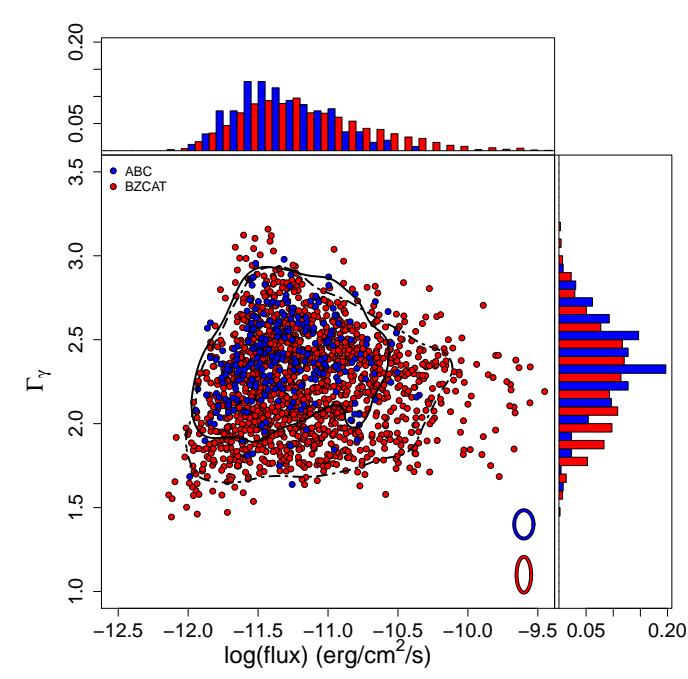

Fig. 19. ABC (blue circles) and Roma-BZCAT (red circles) sources represented on the $\gamma$-ray slope versus $\gamma$-ray flux plot. The ellipses of the relative color indicate the average uncertainties. The black full and dot-dashed lines represent the $90 \% \mathrm{KDE}$ isodensity contours for ABC sources and Roma-BZCAT sources, respectively. On the top and right panels, we present the normalized distributions of the $\gamma$-ray flux and slope for ABC and Roma-BZCAT sources, with the same colors used in the main panel.

tral shape, with BZQs and BZUs being softer in $\gamma$-rays (their $\Gamma_{\gamma}$ distribution peaks at 2.5 and 2.4, respectively) than BZB and BZG (both peaking at 2.0). The $\mathrm{ABC}$ sources are on average softer than average Roma-BZCAT sources, with their $\Gamma_{\gamma}$ distribution peaking at 2.4 and 2.2, respectively. The KS test confirms, however, that the two distributions are completely different, with a $p$-chance, $p=4.0 \times 10^{-13}$, of having been randomly sampled from a common parent distribution. This $p$-chance is, instead, $p=3.7 \times 10^{-2}$, when comparing $\mathrm{ABC}$ sources and BZUs from Roma-BZCAT, indicating that $\mathrm{ABC}$ sources are probably a mixture of different sub-populations possibly dominated by softer FSRQs.

In Fig. 19, we plot ABC and Roma-BZCAT sources with counterparts in 4FGL catalog on a $\gamma$-ray slope versus $\gamma$-ray flux 

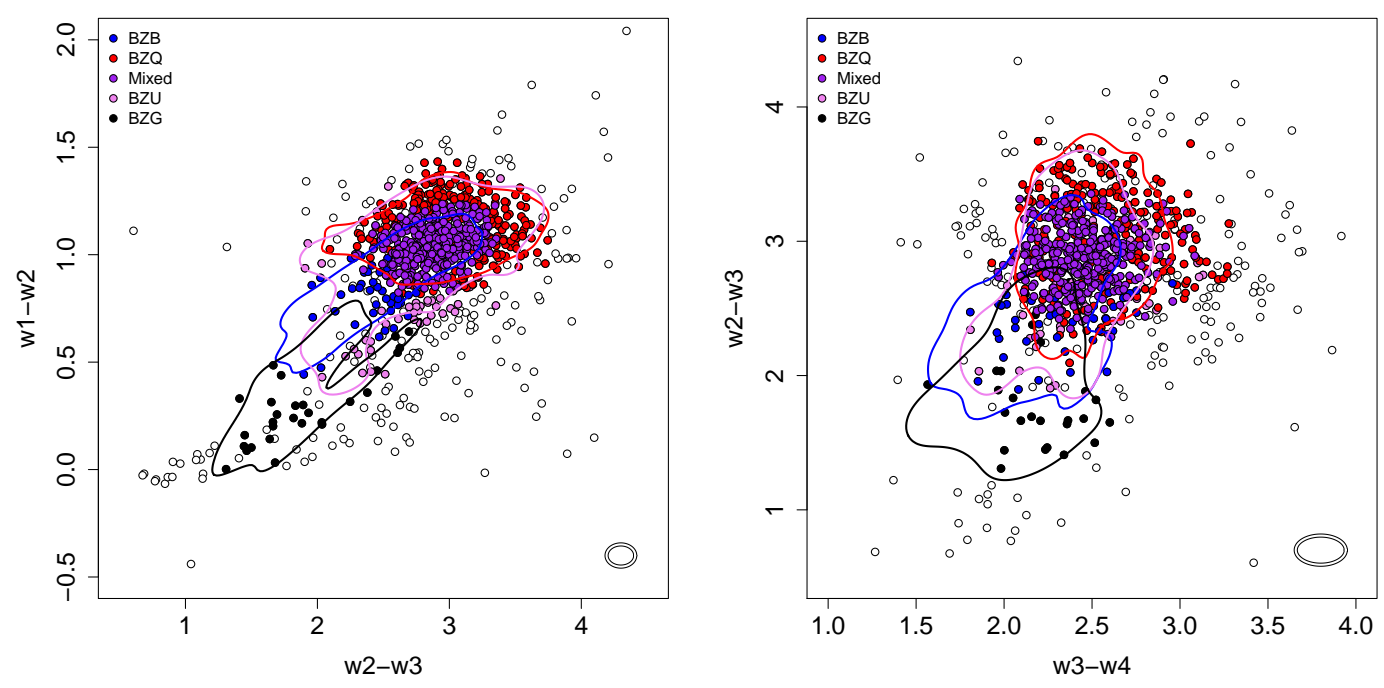

Fig. 20. Left panel: ABC sources with a WISE counterpart detected in all four WISE bands (white circles) represented in the $w 1-w 2$ versus $w 2-w 3$ color-color plane. KDE isodensity contours containing $90 \%$ of the Roma-BZCAT sources detected in $\gamma$-rays (as reported in 4FGL catalog) are presented with lines of different colors for the different Roma-BZCAT subclasses, as indicated in the legend. Filled circles of the respective colors mark the $\gamma$-ray blazar candidates of the different subclasses (see main text). The ellipse indicates the average color uncertainties. Right panel: same as the left panel, but for the $w 2-w 3$ versus $w 3-w 4$ projection.

plot. Again, we note that these two quantities are not independent (since the flux is evaluated from a spectral fit), however this representation is useful for the visualization of the general properties of the sources. On the top and right of this figure, we present the normalized distributions of the $\gamma$-ray flux and slope $\Gamma_{\gamma}$ for $\mathrm{ABC}$ and Roma-BZCAT sources. As noted before, ABC sources are on average softer and dimmer in $\gamma$-rays with respect to the blazar in Roma-BZCAT. This is highlighted by the $90 \% \mathrm{KDE}$ isodensity contours for ABC sources and Roma-BZCAT sources represented with a black full and dot-dashed lines, respectively, that suggest that $\mathrm{ABC}$ sources occupy the same region of the $\gamma$-ray slope versus $\gamma$-ray flux space, although clustering in the softer-dimmer region.

\section{5. $\gamma$-ray blazar candidate selection}

In this section, we make use of the WISE data collected in Sect. 4.2 to select candidate $\gamma$-ray blazars in the ABC sample. As discussed by D'Abrusco et al. (2019), WISE data provide an effective way to select $\gamma$-ray blazars through a comparision of their colors with those of known $\gamma$-ray blazars.

Here we take an approach that is somewhat halfway between those adopted for the compilation of WIBRaLS2 and KDEBLLACS catalogs. First, we selected all Roma-BZCAT sources detected in $\gamma$-rays, that is, with a counterpart in 4FGL catalog (1506 sources) and with a WISE counterpart detected in all four WISE bands (selected as explained in Sect. 4.2), for a total of 1237 sources. Then, for each source subclass (BZB, BZQ, BZU and $\mathrm{BZG}$ ), we evaluated the KDE isodensity contours containing $90 \%$ of the sources in both $w 1-w 2$ versus $w 2-w 3$ and $w 2-w 3$ versus $w 3-w 4$ color-color planes. We then compared the position of the $906 \mathrm{ABC}$ sources with a WISE counterpart detected in all four bands in the two color-color planes, as shown in Fig. 20. Here, the KDE isodensity contours for different RomaBZCAT subclasses are indicated with lines of the relative color indicated in the legend. To select $\gamma$-ray blazar candidates among these ABC sources, we proceeded as follows:

- since the regions occupied by BZBs and BZQs have a significant overlap, if a source is compatible with both the $90 \%$
KDE isodensity contours of BZBs and BZQs on both colorcolor planes, it is classified as $\gamma$-ray blazar candidate of MIXED class,

- if a source is compatible with the $90 \% \mathrm{KDE}$ isodensity contours of BZBs on both color-color planes but not with the $90 \% \mathrm{KDE}$ isodensity contours of BZQs on both color-color planes, it is classified as $\gamma$-ray blazar candidate of BZB class,

- if a source is compatible with the $90 \% \mathrm{KDE}$ isodensity contours of BZQs on both color-color planes but not with the $90 \%$ KDE isodensity contours of BZBs on both color-color planes, it is classified as $\gamma$-ray blazar candidate of BZQ class,

- if a source is compatible with the $90 \% \mathrm{KDE}$ isodensity contours of BZUs on both color-color planes but not with the 90\% KDE isodensity contours of BZBs nor BZQs on both color-color planes, it is classified as $\gamma$-ray blazar candidate of BZU class,

- if a source is compatible with the $90 \% \mathrm{KDE}$ isodensity contours of BZGs on both color-color planes but not with the $90 \% \mathrm{KDE}$ isodensity contours of BZBs, BZQs, or BZUs on both color-color planes, it is classified as $\gamma$-ray blazar candidate of BZG class,

- if a source is not compatible with the $90 \%$ KDE isodensity contours of BZBs, BZQs, BZUs, or BZGs, it is not classified as $\gamma$-ray blazar candidate.

We stress that to consider a source position on the color-color plane compatible with a KDE isodensity contour, we take into account the WISE color uncertainties, that is, the color-color uncertainty ellipse of the source must have an overlap with the isodensity contours. In this way, we select $715 \gamma$-ray blazar candidates, subdivided in 42 candidates BZBs, 247 candidates BZQs, 334 candidates MIXED, 46 candidates BZUs, and 28 candidates BZGs, indicated in Fig. 20 with circles of the respective color. The properties of $\gamma$-ray blazar candidates are summarized in Table C.2, including the WISE counterpart and its blazar candidate classification.

As mentioned before, this selection criterion is intermediate between those presented by D'Abrusco et al. (2019) for the WIBRaLS2 catalog, such that selected sources are detected in all four WISE bands based on their position in the 

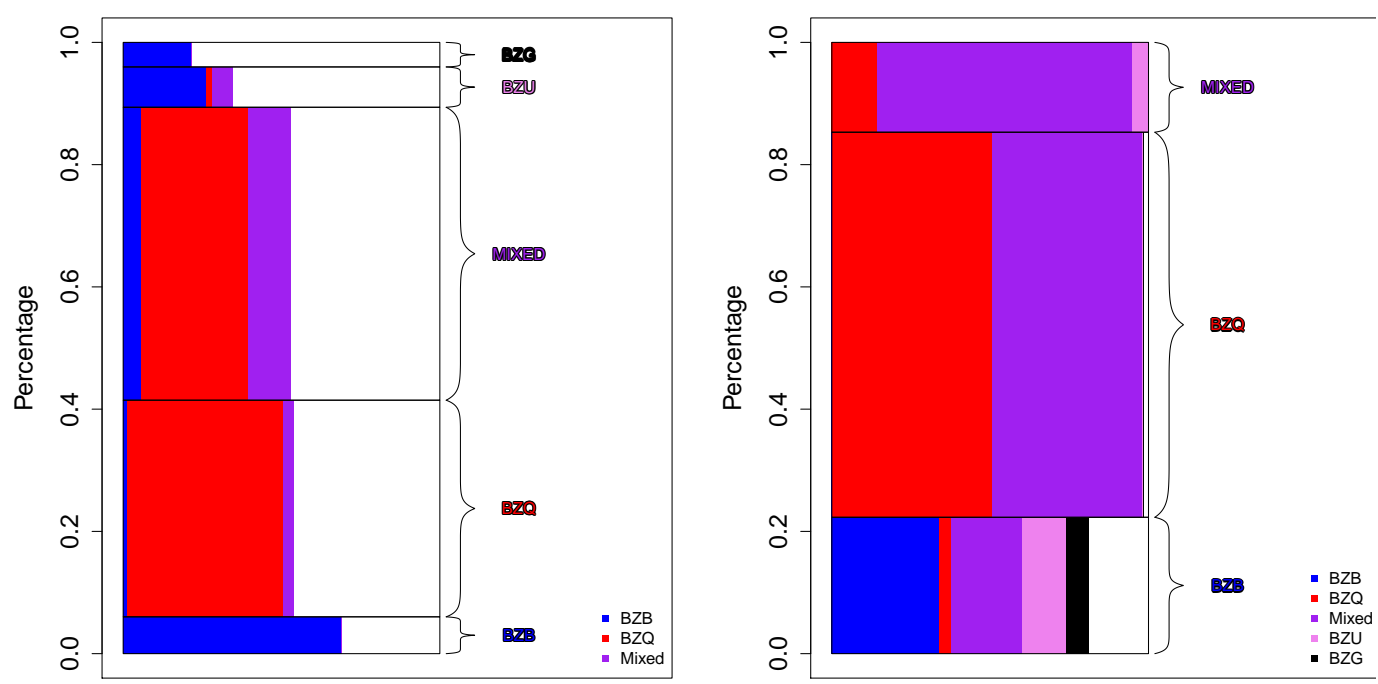

Fig. 21. Left panel: comparison of our classification method and the one proposed by D'Abrusco et al. (2019) for the WIBRaLS2 catalog. The black rectangles represent the $\gamma$-ray blazar candidate classes selected by our method, while the colored bars in each rectangle represent the percentage of sources also classified in WIBRaLS2 catalog. Right panel: same as left panel, but with black rectangles representing the $\gamma$-ray blazar candidate classes of WIBRaLS2 catalog, and with the colored bars representing the percentage of sources also classified in the present work.

three-dimensional principal component space generated by the three independent WISE colors, and for KDEBLLACS, that selects sources detected in the first three WISE bands based on their compatibility with the $90 \% \mathrm{KDE}$ isodensity contours of BZBs in the two-dimensional $w 1-w 2$ versus $w 2-w 3$ colorcolor plane. In addition, the WIBRaLS2 method only selects $\mathrm{BZB}, \mathrm{BZQ}$, and MIXED candidates, while we use our method to select also BZU and BZG candidates. In addition, we note that D'Abrusco et al. (2019) based their selection methods on the third release of the Fermi-LAT catalog, 3FGL, the latest that was available at the time of the publication, while for our method, we used the results of the updated 4FGL. It is for these reasons that the two methods are not equivalent and we expect differences in the classification of the ABC sources.

These differences are summarized in Fig. 21. In the left panel of this figure we present the $715 \mathrm{ABC} \gamma$-ray blazar candidates we selected with our method, 361 of which are also selected in the WIBRaLS2 catalog. For each subclass our classification method (BZB, BZQ, MIXED, BZU and BZG) we indicate with colored rectangles the percentage of sources that have a WIBRaLS2 classification (BZB, BZQ and MIXED). We see that $\sim 70 \%$ of our candidates BZBs are also selected as candidate BZBs in WIBRaLS2, while the remaining $\sim 30 \%$ of this subclass is not classified in WIBRaLS2 catalog. About $\sim 50 \%$ of our BZQ candidates is also classified as BZQ candidate in WIBRaLS2, while three are classified as BZBs and 8 as MIXED in WIBRaLS2. Only $\sim 13 \%$ of the sources we classify as MIXED candidates have the same classification in WIBRaLS2, while $\sim 34 \%$ and $\sim 6 \%$ of these sources are classified as BZQ and BZB candidate in WIBRaLS2, respectively. Finally, $\sim 26 \%$ and $\sim 21 \%$ of the sources that we classify as BZU and BZG, respectively (two classes non-present in WIBRaLS2), are classified as BZB candidates in WIBRaLS2 catalog.

In the right panel of the same figure, we present the reverse comparison, that is, we study how the $361 \mathrm{ABC}$ sources listed in the WIBRaLS2 catalog are classified according to our selection method. The sources classified as BZB candidates in WIBRaLS2 catalog are a mixed bag of different classifications for our method, while $\sim 51 \%$ and $\sim 47 \%$ of the sources classified as BZQ candidates in WIBRaLS2 are classified as candidate BZQs

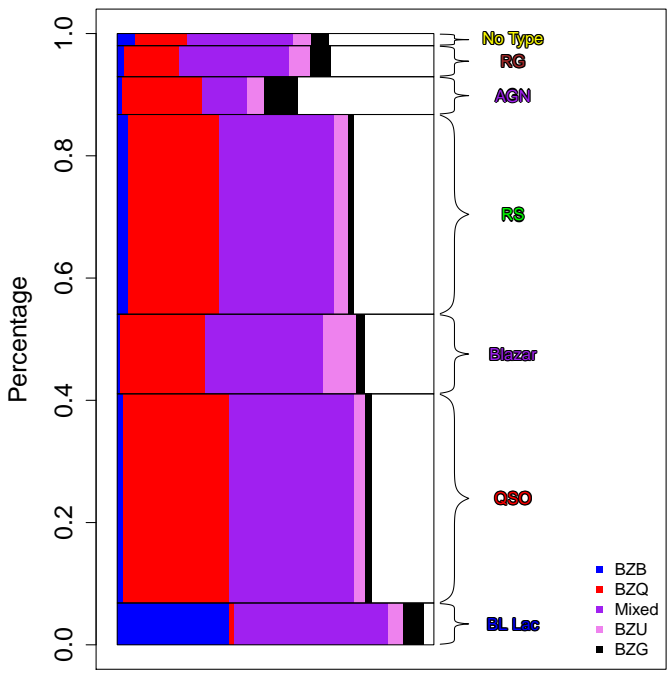

Fig. 22. Summary of the $715 \gamma$-ray blazar candidates selected according to the method presented in Sect. 5. The black rectangles represent the different $\mathrm{ABC}$ source types and the colored bars in each rectangle represent the percentage of each source type classified as $\gamma$-ray blazar candidates of the different classes, with the colors indicated in the legend.

and MIXED according to our method, respectively. Finally, $\sim 14 \%$ and $\sim 80 \%$ of sources classified as candidate MIXED in WIBRaLS2 are classified as BZQs and MIXED candidates in our method.

In Fig. 22, we summarize the results of our $\gamma$-ray blazar candidates selection in terms of $\mathrm{ABC}$ source types. We see that most $\mathrm{BZB}$ candidates belong to the BL Lac source type, while the other source types are mainly composed of candidate BZQs and MIXED candidates, which represent the majority $(\sim 35 \%$ and $\sim 47 \%$ respectively) of our $\gamma$-ray blazar candidates.

To conclude this section, in Fig. 23 we see how the sources for which we have an optical spectroscopic classification (see Sect. 4.1.3) compare with the regions occupied in the WISE color-color diagrams by the different classes of Roma-BZCAT 

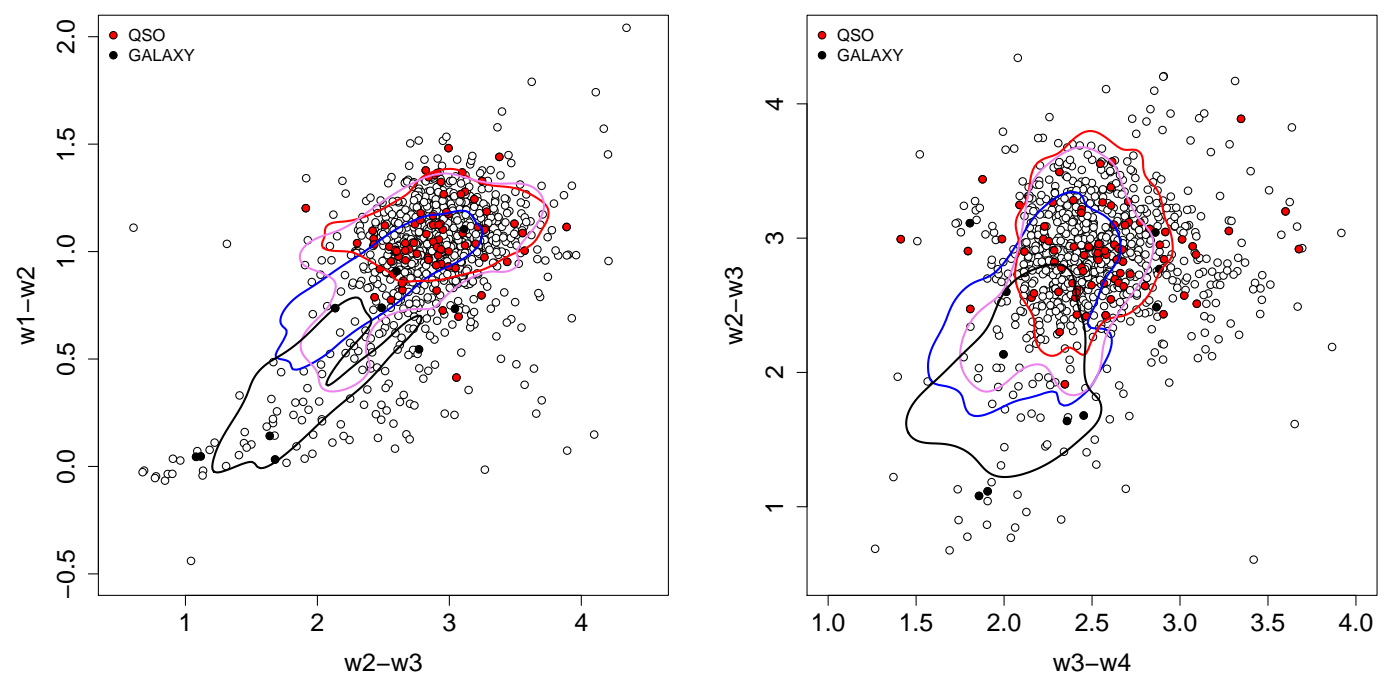

Fig. 23. Left panel: ABC sources with a WISE counterpart detected in all four WISE bands (white circles) represented in the $w 1-w 2$ versus $w 2-w 3$ color-color plane. The same KDE isodensity contours of Fig. 20 are presented with lines of different colors for the different RomaBZCAT subclasses, as indicated in the legend. Filled red and black circles mark sources with an optical spectroscopic classification of QSO and GALAXY, respectively. Right panel: same as the left panel, but for the $w 2-w 3$ versus $w 3-w 4$ projection.

$\gamma$-ray blazars, represented as they are in Fig. 20. Of the 110 sources for which we have an optical spectroscopic classification, 89 have a WISE counterpart detected in all four WISE bands and they are presented in this figure with red circles for sources with an optical spectroscopic classification of QSO, then with black circles for sources with an optical spectroscopic classification of GALAXY. We see that most QSO objects, as expected, lie in the region occupied by BZQs. The GALAXY objects, on the other hand, mostly lie in the region of BZBs especially in the $w 1-w 2$ versus $w 2-w 3$ projection - and in the region of BZGs (whose emission is dominated by the galactic one), extending toward the color-color region occupied by old elliptical galaxies (see e.g. Raiteri et al. 2014), suggesting that these objects are BL Lac objects whose host galaxy dominates the optical emission.

\section{Conclusions}

We built a new catalog of candidate blazars, dubbed ABC, derived from the ALMA Calibrator Catalogue by Bonato et al. (2019). The ABC catalog fills, at least partly, the lack of $|b|<10^{\circ}$ blazars in Roma-BZCAT, providing low Galactic latitude candidate counterparts to unassociated high-energy sources. This is particularly important in the case of rare events, such as detections of high energy neutrinos for which a full exploitation of all-sky data is essential to identify the source population. Some of the ABC sources at low Galactic latitudes may also be useful to verify the Gaia optical reference frame (Mignard et al. 2016).

The ABC catalog contains 1580 sources not included in the Roma-BZCAT. It was cross-matched with Gaia DR2, SDSS DR12, LAMOST DR5, AllWISE, and 4FGL catalogs, finding $805,295,31,1311$, and 259 matches, respectively. These data were used for the classification of our sources and a comparison with the population of known blazars in Roma-BZCAT.

$\mathrm{ABC}$ sources are significantly dimmer than Roma-BZCAT sources in the Gaia $g$ band, while the difference in the Gaia $b-r$ color between the two populations is less pronounced. Also, $\mathrm{ABC}$ sources appear bluer in SDSS than Roma-BZCAT sources, but with low statistical significance. When comparing with the results of Butler \& Bloom (2011), we see that most ABC sources classified as QSO and BL Lac fall into the region of low redshift quasars, with some QSOs entering the regions of higher redshift quasars. Regarding WISE colors, we find that ABC sources are significantly bluer than Roma-BZCAT sources in the $w 2-w 3$ and $w 3-w 4$ colors. In addition, we collected 110 optical spectra in SDSS DR12 and LAMOST DR5, which mostly classify the corresponding sources as QSO (98), while 12 sources resulted in classifications as galactic objects.

A fraction of $\mathrm{ABC}$ sources are located in fields covered by Swift-XRT, Chandra-ACIS and XMM-Newton-EPIC observations. We have retrieved the X-ray archive data and made our own source extraction, achieving the detection of 101, 56 and 55 ABC sources, respectively, extracting spectra for 43, 46, and 51 of them, respectively. Our sources are, on average, similar in X-rays to Roma-BZCAT blazars in terms of count-rates and spectral slopes, implying that our sample is covering the same region of the blazar parameter space in this band. A comparison of $\gamma$-ray properties of ABC sources with Roma-BZCAT blazars has shown that $\mathrm{ABC}$ sources are, on average, dimmer, and their $\gamma$-ray spectra are, on average, softer, consistent with the $\mathrm{ABC}$ containing a significant fraction of FSRQs.

About 57\% (906 out of 1580) of ABC sources are detected in all four WISE bands. This has allowed us to re-examine the selection of $\gamma$-ray blazars by means of mid-IR colors discussed by D'Abrusco et al. (2019). Making use of the KDE contours in the WISE color-color diagrams containing 90\% of WISEdetected 4FGL blazar subclasses, we were able to classify about $80 \%$ (715 out of 906) of the ABC WISE-detected sources as candidate $\gamma$-ray blazar. A comparison with the classification by D'Abrusco et al. (2019) for common sources has shown that these two methods yield different results and may, therefore, be used in a complementary way.

The main properties of the $879 \mathrm{ABC}$ sources for which we were able to collect additional information are summarized in Table 4, including their inclusion in other blazar catalogs (see Sect. 3), the optical spectroscopic classification (see Sect. 4.1.3), the X-ray properties (see Sect. 4.3.4), and the blazar candidate classification (see Sect. 5).

The ABC provides a large sample of candidate blazars that can be investigated both through dedicated optical spectroscopic 
Table 4. Main properties of ABC sources.

\begin{tabular}{|c|c|c|c|c|c|c|c|c|c|c|c|}
\hline Name ALMA & RA & Dec & Object type & Redshift & Type WISE & Type optical & 4FGL & $3 \mathrm{HSP}$ & WIBRaLS2 & KDEBLLACS & $\mathrm{X}$-ray \\
\hline J0002-2153 & 00:02:11.98 & $-21: 53: 09.87$ & BL Lac & & MIXED & & & & & & \\
\hline J0006-2955 & 00:06:01.12 & $-29: 55: 50.10$ & QSO & 0.683 & BZU & & & & & & \\
\hline J0008-3945 & 00:08:09.20 & $-39: 45: 22.96$ & RS & 1.9 & MIXED & & $\mathrm{BCU}$ & & BZQ & & \\
\hline $\mathrm{J} 0011+0823$ & $00: 11: 35.27$ & $+08: 23: 55.59$ & QSO & 1.35 & MIXED & QSO & & & BZQ & & \\
\hline J0011-4105 & $00: 11: 52.40$ & $-41: 05: 45.17$ & $\mathrm{RS}$ & & MIXED & & & & & & \\
\hline J0011-8443 & $00: 11: 45.91$ & $-84: 43: 20.01$ & RS & 0.6 & MIXED & & & & BZQ & & \\
\hline J0011-8706 & 00:11:51.48 & $-87: 06: 25.45$ & RG & 3.292 & & & & & & & det \\
\hline J0019-5641 & 00:19:26.61 & $-56: 41: 42.46$ & RS & & & & $\mathrm{BCU}$ & & & & \\
\hline J0024-0811 & $00: 24: 00.67$ & $-08: 11: 10.05$ & QSO & 2.067 & BZQ & QSO & & & BZQ & & \\
\hline J0024-4202 & 00:24:42.99 & $-42: 02: 03.95$ & QSO & 0.937 & BZQ & & & & BZQ & & \\
\hline
\end{tabular}

Notes. For each source we list the name (Name ALMA), the coordinates (RA, Dec), the ABC source type (Object type), the redshift (Redshift), the blazar candidate class obtained from WISE colors (Type WISE), the source type obtained from optical spectra (Type optical), the source class listed in the 4FGL catalog (4FGL), if the source is present in the 3HSP catalog (3HSP), the blazar candidate class as reported in the WIBRaLS2 catalog (WIBRaLS2), if the source is present in the KDEBLLACS catalog (KDEBLLACS), and the X-ray information (X-ray), namely a source detection (det), a power-law spectrum (PL) or a more complex spectrum (COMPLEX). The full table is available in electronic form at the CDS.

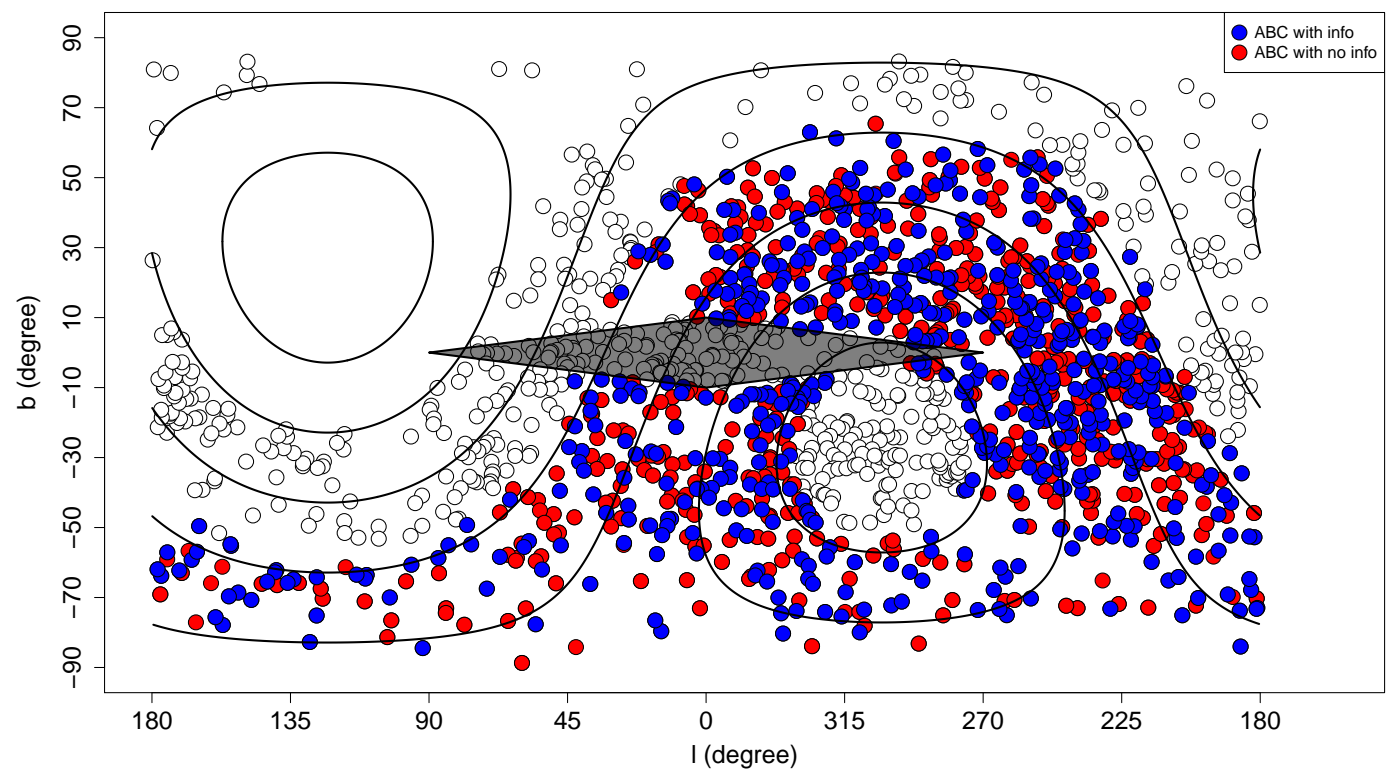

Fig. 24. White circles: Galactic coordinates of the ABC sources. Colored circles: ABC sources falling in the area covered by the planned WFD survey, with the exception of the Galactic center indicated with a dark lozenge. In particular, blue circles represent ABC sources for which we were able to collect additional information (see Table 4), while the red circles represent ABC sources for which we could not collect additional information. The black lines represent equatorial coordinates with declinations from $-60^{\circ}$ to $60^{\circ}$ with increment of $20^{\circ}$.

observation campaigns or through repeated photometric observations for variability studies. An ideal tool for performing the latter investigation will be the ten-year Legacy Survey of Space and Time (LSST) which, starting from 2022, will be performed at the Vera C. Rubin Observatory (Ivezić et al. 2019). The LSST will repeatedly scan the whole southern sky, providing multi-epoch observations in six optical photometric bands (ugrizy) for more than 35 billions objects. The main LSST survey will be the Wide, Fast, Deep (WFD) survey, observing the sky between $-65^{\circ}$ and $+5^{\circ}$ of declination, with an observation cadence of $\sim 3$ days. In Fig. 24 we show the ABC sources in Galactic coordinates with white circles. The colored circles represent the $\mathrm{ABC}$ sources falling in the area covered by the planned WFD survey, with the exception of the Galactic center indicated with a dark lozenge. In particular, in the WFD survey area, we have $556 \mathrm{ABC}$ sources with additional information contained in Table 4 (blue circles) and 529 ABC sources without additional information (red circles). Among the 125
ABC sources in the WFD survey area with an SDSS DR12 counterpart, 114 (more than 90\%) have a $r$ magnitude between the median single-visit $5 \sigma$ point sources depth of $r=24.16$ for the planned WFD survey and the nominal LSST saturation limit of $r \sim 16$ for 15 s exposures ${ }^{14}$. The data collected by the WFD survey will therefore provide a key tool for the investigation of the possible blazar nature of these sources.

Acknowledgements. We thank our anonymous referee for useful comments and suggestions. This project has received funding from the European Union'S Horizon 2020 research and innovation programme under the Marie SkłodowskaCurie grant agreement NO 664931. MB acknowledges support from INAF under PRIN SKA/CTA FORECaST and from the Ministero degli Affari Esteri della Cooperazione Internazionale - Direzione Generale per la Promozione del Sistema Paese Progetto di Grande Rilevanza ZA18GR02. This work has made use of data from the European Space Agency (ESA) mission Gaia (https://www.cosmos.esa.int/gaia), processed by the Gaia

\footnotetext{
${ }^{14}$ See the white paper on the LSST Observing Strategy.
} 
Data Processing and Analysis Consortium (DPAC, https://www.cosmos. esa.int/web/gaia/dpac/consortium). Funding for the DPAC has been provided by national institutions, in particular the institutions participating in the Gaia Multilateral Agreement. Funding for the Sloan Digital Sky Survey IV has been provided by the Alfred P. Sloan Foundation, the US Department of Energy Office of Science, and the Participating Institutions. SDSS-IV acknowledges support and resources from the Center for High-Performance Computing at the University of Utah. The SDSS website is www.sdss.org. SDSS-IV is managed by the Astrophysical Research Consortium for the Participating Institutions of the SDSS Collaboration including the Brazilian Participation Group, the Carnegie Institution for Science, Carnegie Mellon University, the Chilean Participation Group, the French Participation Group, Harvard-Smithsonian Center for Astrophysics, Instituto de Astrofísica de Canarias, The Johns Hopkins University, Kavli Institute for the Physics and Mathematics of the Universe (IPMU)/University of Tokyo, the Korean Participation Group, Lawrence Berkeley National Laboratory, Leibniz Institut für Astrophysik Potsdam (AIP), Max-Planck-Institut für Astronomie (MPIA Heidelberg), Max-Planck-Institut für Astrophysik (MPA Garching), Max-Planck-Institut für Extraterrestrische Physik (MPE), National Astronomical Observatories of China, New Mexico State University, New York University, University of Notre Dame, Observatário Nacional/MCTI, The Ohio State University, Pennsylvania State University, Shanghai Astronomical Observatory, United Kingdom Participation Group Universidad Nacional Autónoma de México, University of Arizona, University of Colorado Boulder, University of Oxford, University of Portsmouth, University of Utah, University of Virginia, University of Washington, University of Wisconsin, Vanderbilt University, and Yale University. This research has made use of the SIMBAD database, operated at CDS, Strasbourg, France. Guoshoujing Telescope (the Large Sky Area Multi-Object Fiber Spectroscopic Telescope LAMOST) is a National Major Scientific Project built by the Chinese Academy of Sciences. Funding for the project has been provided by the National Developmen and Reform Commission. LAMOST is operated and managed by the National Astronomical Observatories, Chinese Academy of Sciences. This publication makes use of data products from the Wide-field Infrared Survey Explorer, which is a joint project of the University of California, Los Angeles, and the Jet Propulsion Laboratory/California Institute of Technology, funded by the National Aeronautics and Space Administration. We acknowledge the use of public data from the Swift data archive. This research has made use of data obtained from the Chandra Data Archive. This research has made use of observations obtained with XMM-Newton, an ESA science mission with instruments and contributions directly funded by ESA Member States and NASA. This research has made use of data and software provided by the High Energy Astrophysics Science Archive Research Center (HEASARC), which is a service of the Astrophysics Science Division at NASA/GSFC. This research has made use of software provided by the Chandra X-ray Center (CXC) in the application packages CIAO, ChIPS, an Sherpa. This research has made use of the TOPCAT software (Taylor 2005).

\section{References}

Abdo, A. A., Ackermann, M., Ajello, M., et al. 2010a, ApJS, 188, 405 Abdo, A. A., Ackermann, M., Ajello, M., et al. 2010b, ApJ, 715, 429 Abdollahi, S., Acero, F., Ackermann, M., et al. 2020, ApJS, 247, 33 Abeysekara, A. U., Benbow, W., Bird, R., et al. 2020, ApJ, 890, 97 Acero, F., Ackermann, M., Ajello, M., et al. 2015, ApJS, 218, 23 Ackermann, M., Ajello, M., Allafort, A., et al. 2011, ApJ, 743, 171 Ackermann, M., Ajello, M., Atwood, W. B., et al. 2015, ApJ, 810, 14 Adelman-McCarthy, J. K., et al. 2009, VizieR Online Data Catalog: II/294 Aharonian, F., Akhperjanian, A. G., Anton, G., et al. 2009, A\&A, 502, 749 Alam, S., Albareti, F. D., Allende Prieto, C., et al. 2015, ApJS, 219, 12 Aleksić, J., Alvarez, E. A., Antonelli, L. A., et al. 2012, A\&A, 544, A142 Aliu, E., Aune, T., Beilicke, M., et al. 2011, ApJ, 742, 127

Allen, J. T., Hewett, P. C., Maddox, N., et al. 2011, MNRAS, 410, 860 Álvarez Crespo, N., Masetti, N., Ricci, F., et al. 2016, AJ, 151, 32 Bailer-Jones, C. A. L., Fouesneau, M., \& Andrae, R. 2019, MNRAS, 490, 5615 Baumgartner, W. H., Tueller, J., Markwardt, C. B., et al. 2013, ApJS, 207, 19 Best, P. N., Kauffmann, G., Heckman, T. M., et al. 2005, MNRAS, 362, 9 Bhagwan, J., Gupta, A. C., Papadakis, I. E., et al. 2016, New Astron., 44, 21 Blandford, R. D., \& Rees, M. J. 1978, BL Lac Objects (Pittsburgh: University of Pittsburgh), 328

Böhringer, H., Schuecker, P., Guzzo, L., et al. 2004, A\&A, 425, 367 Bonaldi, A., Bonavera, L., Massardi, M., et al. 2013, MNRAS, 428, 1845 Bonato, M., Liuzzo, E., Herranz, D., et al. 2019, MNRAS, 485, 1188 Brookes, M. H., Best, P. N., Rengelink, R., et al. 2006, MNRAS, 366, 1265 Brookes, M. H., Best, P. N., Peacock, J. A., et al. 2008, MNRAS, 385, 1297 Butler, N. R., \& Bloom, J. S. 2011, AJ, 141, 93

Capalbi, M., Perri, M., Saija, B., Tamburelli, F., \& Angelini, L. 2005, The Swift XRT Data Reduction Guide, Technical Report 1.2, http://swift.gsfc. nasa.gov/analysis/xrt_swguide_v1_2.pdf
Capetti, A., \& Raiteri, C. M. 2015, A\&A, 580, A73

Carnerero, M. I., Raiteri, C. M., Villata, M., et al. 2017, MNRAS, 472, 3789

Carter, J. A., \& Read, A. M. 2007, A\&A, 464, 1155

Chang, Y.-L., Arsioli, B., Giommi, P., et al. 2019, A\&A, 632, A77

Chiaro, G., Meyer, M., Di Mauro, M., et al. 2019, ApJ, 887, 104

Comastri, A., Fossati, G., Ghisellini, G., et al. 1997, ApJ, 480, 534

Condon, J. J., Cotton, W. D., Greisen, E. W., et al. 1998, AJ, 115, 1693

Crook, A. C., Huchra, J. P., Martimbeau, N., et al. 2007, ApJ, 655, 790

Cui, X.-Q., Zhao, Y.-H., Chu, Y.-Q., et al. 2012, Res. Astron. Astrophys., 12, 1197

Cusumano, G., La Parola, V., Segreto, A., et al. 2010, A\&A, 524, A64

Cutri, R. M., Wright, E. L., Conrow, T., et al. 2013, Explanatory Supplement to the AllWISE Data Release Products

D'Abrusco, R., Massaro, F., Ajello, M., et al. 2012, ApJ, 748, 68

D'Abrusco, R., Massaro, F., Paggi, A., et al. 2013, ApJS, 206, 12

D'Abrusco, R., Massaro, F., Paggi, A., et al. 2014, ApJS, 215, 14

D’Abrusco, R., Álvarez Crespo, N., Massaro, F., et al. 2019, ApJS, 242, 4

Dai, X., Bregman, J. N., \& Kochanek, C. S. 2012, Am. Astron. Soc. Meet. Abstr., 219, 415.06

D’Elia, V., Perri, M., Puccetti, S., et al. 2013, A\&A, 551, A142

de Menezes, R., Amaya-Almazán, R. A., Marchesini, E. J., et al. 2020, Ap\&SS,

365,12

Dermer, C. D., \& Schlickeiser, R. 1993, ApJ, 416, 458

Dermer, C. D., Finke, J. D., Krug, H., et al. 2009, ApJ, 692, 32

Di Mauro, M., Manconi, S., Zechlin, H.-S., et al. 2018, ApJ, 856, 106

Donato, D., Ghisellini, G., Tagliaferri, G., et al. 2001, A\&A, 375, 739

Edelson, R., \& Malkan, M. 2012, ApJ, 751, 52

Elvis, M., Plummer, D., Schachter, J., et al. 1992, ApJS, 80, 257

Fidelis, V. V., Yakubovskyi, D. A., \& Voytkova, Y. V. 2009, Astron. Lett., 35, 579

Fitzpatrick, E. L., \& Massa, D. 2007, ApJ, 663, 320

Freeman, P., Doe, S., \& Siemiginowska, A. 2001, Proc. SPIE, 4477, 76

Frew, D. J., Bojičić, I. S., \& Parker, Q. A. 2013, MNRAS, 431, 2

Fruscione, A., McDowell, J. C., Allen, G. E., et al. 2006, Proc. SPIE, 6270, $62701 \mathrm{~V}$

Gaia Collaboration (Prusti, T., et al.) 2016a, A\&A, 595, A1

Gaia Collaboration (Brown, A. G. A., et al.) 2016b, A\&A, 595, A2

Gaia Collaboration (Brown, A. G. A., et al.) 2018, A\&A, 616, A1

Garrappa, S., Buson, S., Franckowiak, A., et al. 2019, ApJ, 880, 103

Gehrels, N. 1986, ApJ, 303, 336

Gentile Fusillo, N. P., Gänsicke, B. T., \& Greiss, S. 2015a, MNRAS, 448, 2260

Gentile Fusillo, N. P., Rebassa-Mansergas, A., Gänsicke, B. T., et al. 2015b, MNRAS, 452, 765

Giommi, P., \& Padovani, P. 1994, MNRAS, 268, L51

Giommi, P., Polenta, G., Lähteenmäki, A., et al. 2012, A\&A, 541, A160

Giommi, P., Padovani, P., \& Polenta, G. 2013, MNRAS, 431, 1914

Gregory, P. C., Scott, W. K., Douglas, K., et al. 1996, ApJS, 103, 427

Guzzo, L., Schuecker, P., Böhringer, H., et al. 2009, A\&A, 499, 357

Healey, S. E., Romani, R. W., Cotter, G., et al. 2008, ApJS, 175, 97

H.E.S.S. Collaboration (Abramowski, A., et al.) 2013, A\&A, 552, A118

Hogan, B. S., Lister, M. L., Kharb, P., et al. 2011, ApJ, 730, 92

IceCube Collaboration (Aartsen, M. G., et al.) 2018, Science, 361, eaat1378

Ighina, L., Caccianiga, A., Moretti, A., et al. 2019, MNRAS, 489, 2732

Inoue, S., \& Takahara, F. 1996, ApJ, 463, 555

Ivezić, Ž., Kahn, S. M., Tyson, J. A., et al. 2019, ApJ, 873, 111

Jorstad, S. G., \& Marscher, A. P. 2004, ApJ, 614, 615

Kalberla, P. M. W., Burton, W. B., Hartmann, D., et al. 2005, A\&A, 440, 775

Kalita, N., Gupta, A. C., Wiita, P. J., et al. 2015, MNRAS, 451, 1356

Koay, J. Y., Macquart, J.-P., Rickett, B. J., et al. 2011, AJ, 142, 108

Kolmogorov, A. N. 1933, Giornale dell'Instituto Italiano degli Attuari, 4, 83

Krawczyk, C. M., Richards, G. T., Mehta, S. S., et al. 2013, ApJS, 206, 4

Krimm, H. A., Holland, S. T., Corbet, R. H. D., et al. 2013, ApJS, 209, 14

Krishnan, V., Ellingsen, S. P., Reid, M. J., et al. 2017, MNRAS, 465, 1095

Landoni, M., Massaro, F., Paggi, A., et al. 2015, AJ, 149, 163

Landt, H., Padovani, P., Perlman, E. S., et al. 2001, MNRAS, 323, 757

Lanyi, G. E., Boboltz, D. A., Charlot, P., et al. 2010, AJ, 139, 1695

Laurent-Muehleisen, S. A., Kollgaard, R. I., Feigelson, E. D., et al. 1999, ApJ, 525,127

Lavaux, G., \& Hudson, M. J. 2011, MNRAS, 416, 2840

Lefaucheur, J., \& Pita, S. 2017, A\&A, 602, A86

Li, J., An, T., Shen, Z.-Q., et al. 2010, ApJ, 720, L56

Lyu, Y., \& Liu, X. 2016, MNRAS, 463, 24

Mahony, E. K., Sadler, E. M., Croom, S. M., et al. 2011, MNRAS, 417, 2651

Makarov, V. V., Frouard, J., Berghea, C. T., et al. 2017, ApJ, 835, L30

Malizia, A., Bassani, L., Bazzano, A., et al. 2012, MNRAS, 426, 1750

Marchesini, E. J., Andruchow, I., Cellone, S. A., et al. 2016a, A\&A, 591, A21

Marchesini, E. J., Masetti, N., Chavushyan, V., et al. 2016b, A\&A, 596, A10

Marchesini, E. J., Paggi, A., Massaro, F., et al. 2019, A\&A, 631, A150 
Marscher, A. P., \& Jorstad, S. G. 2011, ApJ, 729, 26

Marsden, D., Gralla, M., Marriage, T. A., et al. 2014, MNRAS, 439, 1556

Maselli, A., Massaro, E., Nesci, R., et al. 2010, A\&A, 512, A74

Maselli, A., Massaro, F., Cusumano, G., et al. 2013, ApJS, 206, 17

Massardi, M., Ekers, R. D., Murphy, T., et al. 2008, MNRAS, 384, 775

Massaro, F., Tramacere, A., Cavaliere, A., et al. 2008, A\&A, 478, 395

Massaro, E., Giommi, P., Leto, C., et al. 2009, A\&A, 495, 691

Massaro, F., Paggi, A., Elvis, M., et al. 2011a, ApJ, 739, 73

Massaro, F., Paggi, A., \& Cavaliere, A. 2011b, ApJ, 742, L32

Massaro, F., D'Abrusco, R., Tosti, G., et al. 2012, ApJ, 752, 61

Massaro, F., D’Abrusco, R., Paggi, A., et al. 2013, ApJS, 209, 10

Massaro, F., Thompson, D. J., \& Ferrara, E. C. 2015a, A\&ARv, 24, 2

Massaro, E., Maselli, A., Leto, C., et al. 2015b, Ap\&SS, 357, 75

Massaro, F., D’Abrusco, R., Landoni, M., et al. 2015c, ApJS, 217, 2

Mauch, T., Murphy, T., Buttery, H. J., et al. 2003, MNRAS, 342, 1117

Megeath, S. T., Gutermuth, R., Muzerolle, J., et al. 2012, AJ, 144, 192

Meusinger, H., Hinze, A., \& de Hoon, A. 2011, A\&A, 525, A37

Mignard, F., Klioner, S., Lindegren, L., et al. 2016, A\&A, 595, A5

Miller, B. P., Brandt, W. N., Schneider, D. P., et al. 2011, ApJ, 726, 20

Monroe, T. R., Prochaska, J. X., Tejos, N., et al. 2016, AJ, 152, 25

Moretti, A., Campana, S., Mineo, T., et al. 2005, Proc. SPIE, 5898, 360

Moretti, A., Perri, M., Capalbi, M., et al. 2007, Proc. SPIE, 6688, 66880G

Moretti, A., Vattakunnel, S., Tozzi, P., et al. 2012, A\&A, 548, A87

Nebot Gómez-Morán, A., Motch, C., Barcons, X., et al. 2013, A\&A, 553, A12

Nevalainen, J., Markevitch, M., \& Lumb, D. 2005, ApJ, 629, 172

Nolan, P. L., Abdo, A. A., Ackermann, M., et al. 2012, ApJS, 199, 31

Pâris, I., Petitjean, P., Aubourg, É., et al. 2014, A\&A, 563, A54

Pâris, I., Petitjean, P., Ross, N. P., et al. 2017, A\&A, 597, A79

Padovani, P., \& Giommi, P. 1995, ApJ, 444, 567

Paggi, A., Massaro, F., D’Abrusco, R., et al. 2013, ApJS, 209, 9

Pasetto, A., Kraus, A., Mack, K.-H., et al. 2016, A\&A, 586, A117

Peña-Herazo, H. A., Massaro, F., Chavushyan, V., et al. 2019, Ap\&SS, 364, 85

Perlman, E. S. 2000, Am. Inst. Phys. Conf. Ser., 53, 4827

Perlman, E. S., Padovani, P., Giommi, P., et al. 1998, AJ, 115, 1253

Perlman, E. S., Schachter, J. F., \& Stocke, J. T. 1999, Am. Astron. Soc. Meet. Abstr., 195, 16.01

Perri, M., Maselli, A., Giommi, P., et al. 2007, A\&A, 462, 889

Petrov, L., \& Taylor, G. B. 2011, AJ, 142, 89

Petrov, L., Mahony, E. K., Edwards, P. G., et al. 2013, MNRAS, 432, 1294

Pierre, M., Pacaud, F., Adami, C., et al. 2016, A\&A, 592, A1

Planck Collaboration XXVIII. 2014, A\&A, 571, A28

Plotkin, R. M., Anderson, S. F., Brandt, W. N., et al. 2012, ApJ, 745, L27

Prochaska, J. X., Hennawi, J. F., \& Herbert-Fort, S. 2008, ApJ, 675, 1002
Puccetti, S., Capalbi, M., Giommi, P., et al. 2011, A\&A, 528, A122 Pursimo, T., Ojha, R., Jauncey, D. L., et al. 2013, ApJ, 767, 14 Pushkarev, A. B., \& Kovalev, Y. Y. 2012, A\&A, 544, A34 Raiteri, C. M., \& Capetti, A. 2016, A\&A, 587, A8

Raiteri, C. M., Villata, M., Kadler, M., et al. 2006, A\&A, 452, 845

Raiteri, C. M., Villata, M., Aller, M. F., et al. 2011, A\&A, 534, A87

Raiteri, C. M., Villata, M., D’Ammando, F., et al. 2013, MNRAS, 436, 1530

Raiteri, C. M., Villata, M., Carnerero, M. I., et al. 2014, MNRAS, 442, 629

Raiteri, C. M., Villata, M., Carnerero, M. I., et al. 2019, MNRAS, 489, 1837

Rau, A., Schady, P., Greiner, J., et al. 2012, A\&A, 538, A26

Rieger, F. M., Kirk, J. G., \& Mastichiadis, A. 2000, ArXiv e-prints [arXiv:astro-ph/0005479]

Ricci, R., Sadler, E. M., Ekers, R. D., et al. 2004, MNRAS, 354, 305

Ricci, F., Massaro, F., Landoni, M., et al. 2015, AJ, 149, 160

Riley, A. H., Strigari, L. E., Porter, T. A., et al. 2019, ApJ, 878, 8

Rochais, T., Singh, V., Chick, W., et al. 2017, MNRAS, 464, 553

Sarazin, F., Anchordoqui, L., Beatty, J., et al. 2019, BAAS, 51, 93

Schlafly, E. F., \& Finkbeiner, D. P. 2011, ApJ, 737, 103

Smirnov, N. V. 1939, Bull. Moscow Univ., 2, 3

Sowards-Emmerd, D., Romani, R. W., Michelson, P. F., et al. 2005, ApJ, 626, 95

Stickel, M., Padovani, P., Urry, C. M., et al. 1991, ApJ, 374, 431

Stocke, J. T., \& Rector, T. A. 1997, ApJ, 489, L17

Sun, J., \& Shen, Y. 2015, ApJ, 804, L15

Szabo, T., Pierpaoli, E., Dong, F., et al. 2011, ApJ, 736, 21

Tavecchio, F., Maraschi, L., Wolter, A., et al. 2007, ApJ, 662, 900

Taylor, M. B. 2005, Astronomical Data Analysis Software and Systems XIV

(San Francisco: Astronomical Society of the Pacific), 29

Tempel, E., Kipper, R., Tamm, A., et al. 2016, A\&A, 588, A14

Titov, O., \& Malkin, Z. 2009, A\&A, 506, 1477

Titov, O., Lambert, S. B., \& Gontier, A.-M. 2011, A\&A, 529, A91

Titov, O., Pursimo, T., Johnston, H. M., et al. 2017, AJ, 153, 157

Toba, Y., Oyabu, S., Matsuhara, H., et al. 2014, ApJ, 788, 45

Tramacere, A., Giommi, P., Massaro, E., et al. 2007, A\&A, 467, 501 Urry, C. M., \& Padovani, P. 1995, PASP, 107, 803

van Velzen, S., Falcke, H., Schellart, P., et al. 2012, A\&A, 544, A18

Véron-Cetty, M.-P., \& Véron, P. 2010, A\&A, 518, A10

Von Der Linden, A., Best, P. N., Kauffmann, G., et al. 2007, MNRAS, 379, 867

Wenger, M., Ochsenbein, F., Egret, D., et al. 2000, A\&AS, 143, 9

White, R. L., Becker, R. H., Helfand, D. J., et al. 1997, ApJ, 475, 479

Wright, A. E., Griffith, M. R., Burke, B. F., et al. 1994, ApJS, 91, 111

Wright, E. L., Eisenhardt, P. R. M., Mainzer, A. K., et al. 2010, AJ, 140, 1868

Wylezalek, D., Galametz, A., Stern, D., et al. 2013, ApJ, 769, 79

Yuan, Z. S., Han, J. L., \& Wen, Z. L. 2016, MNRAS, 460, 3669

Zickgraf, F.-J., Engels, D., Hagen, H.-J., et al. 2003, A\&A, 406, 535 


\section{Appendix A: X-ray spectra}

In this appendix we collect the results of the spectral fitting discussed in Sect. 4.3.4. In particular, in Figs. A.1-A.3 we show the spectra fitted with a power-law model, while in Figs. A.4-A.6 we show spectra fitted with the models listed in Table 3.
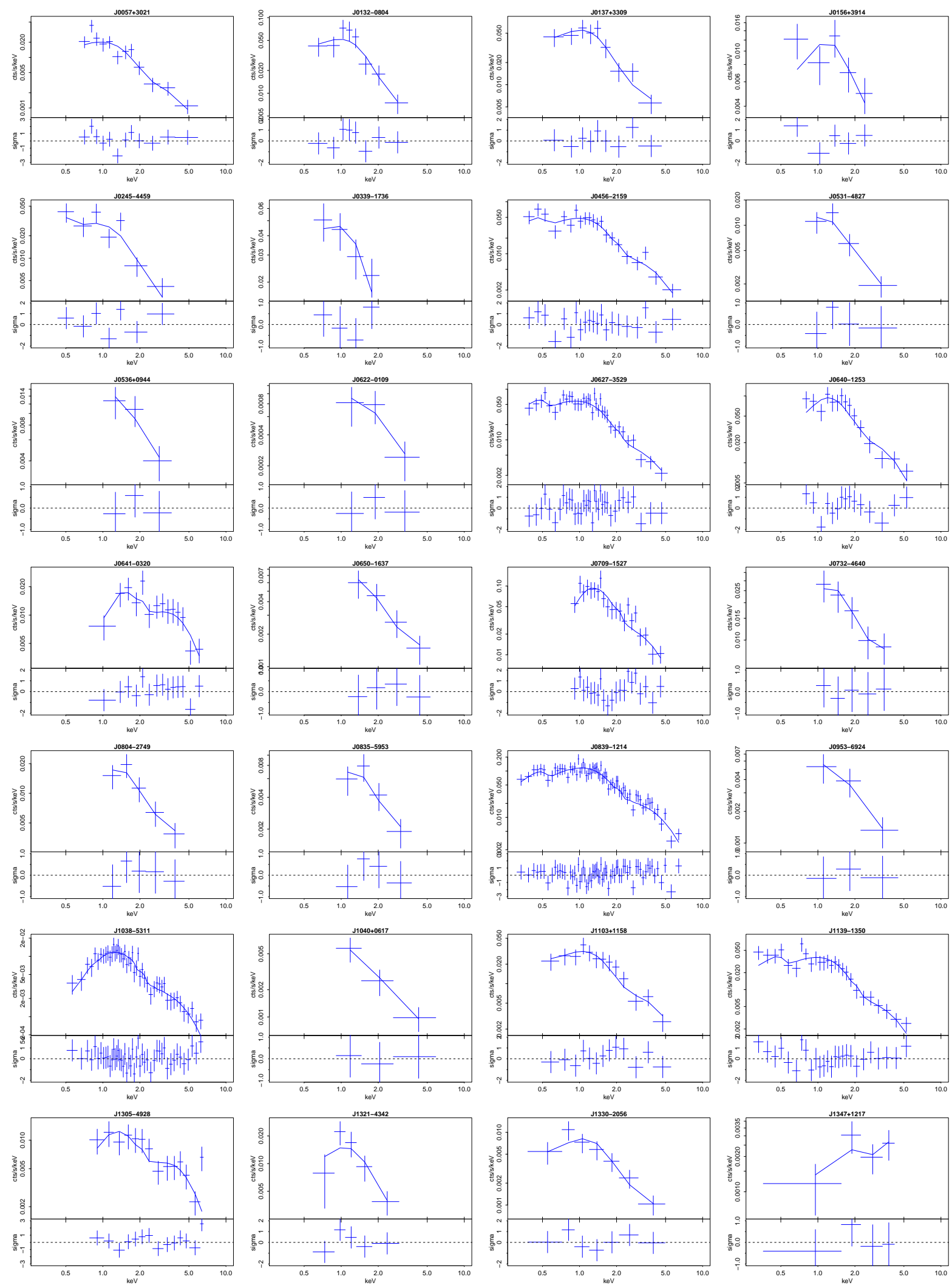

Fig. A.1. Swift-XRT spectra with their best-fit power law models (upper panels) and residuals (lower panels). 
A\&A 641, A62 (2020)
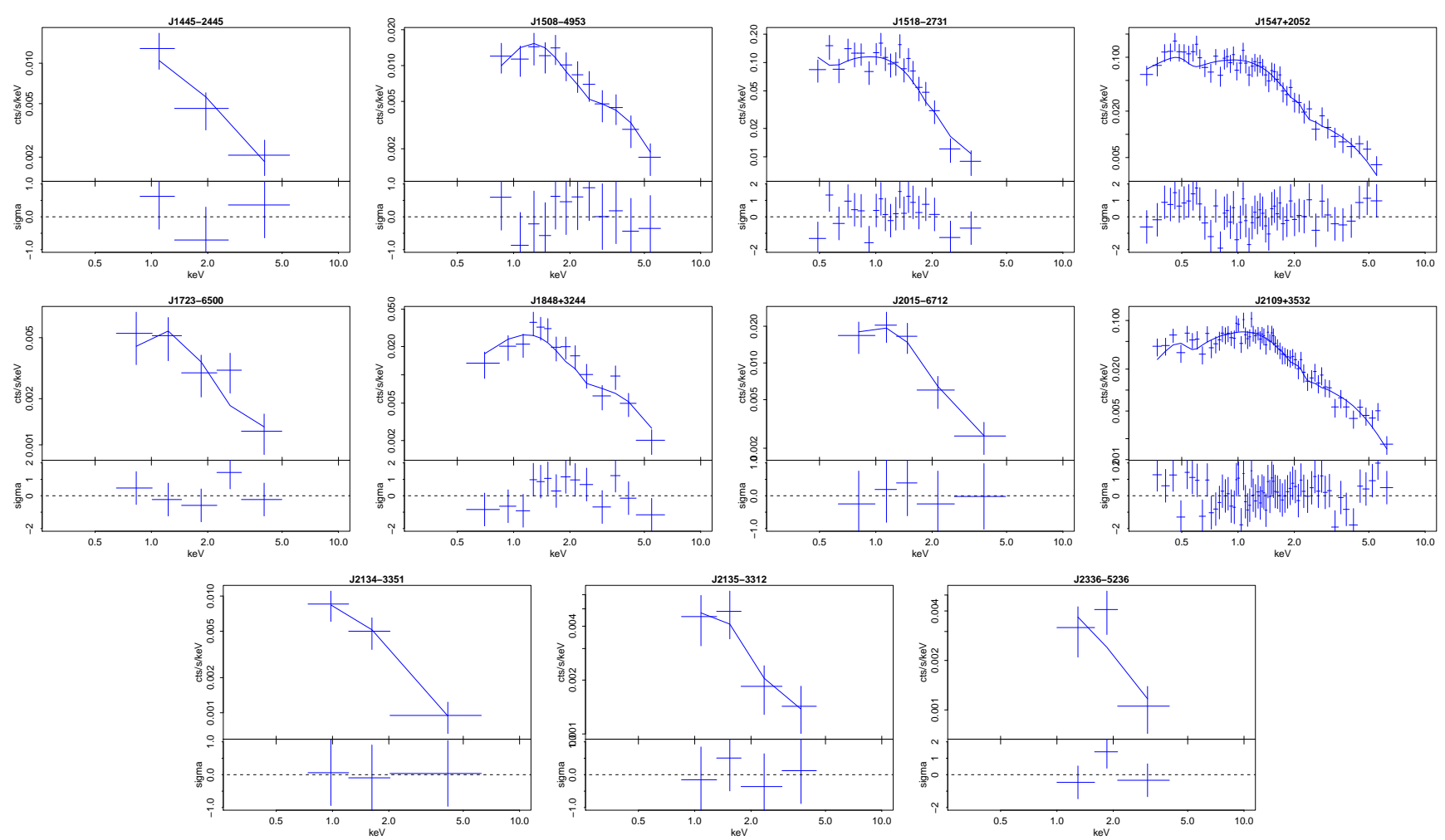

Fig. A.1. continued. 
A. Paggi et al.: A new multiwavelength census of blazars
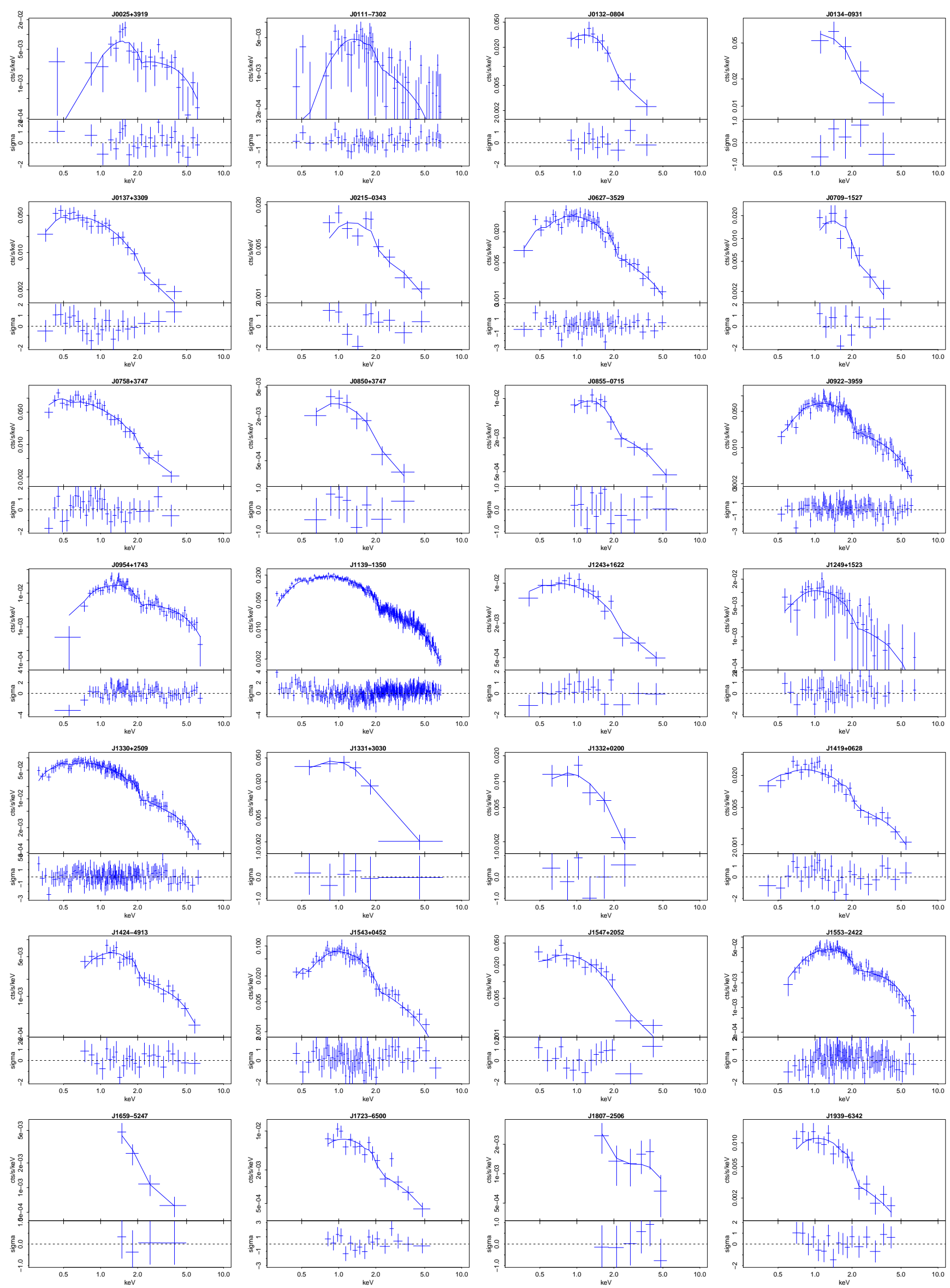

Fig. A.2. Chandra-ACIS spectra with their best-fit power law models (upper panels) and residuals (lower panels). 

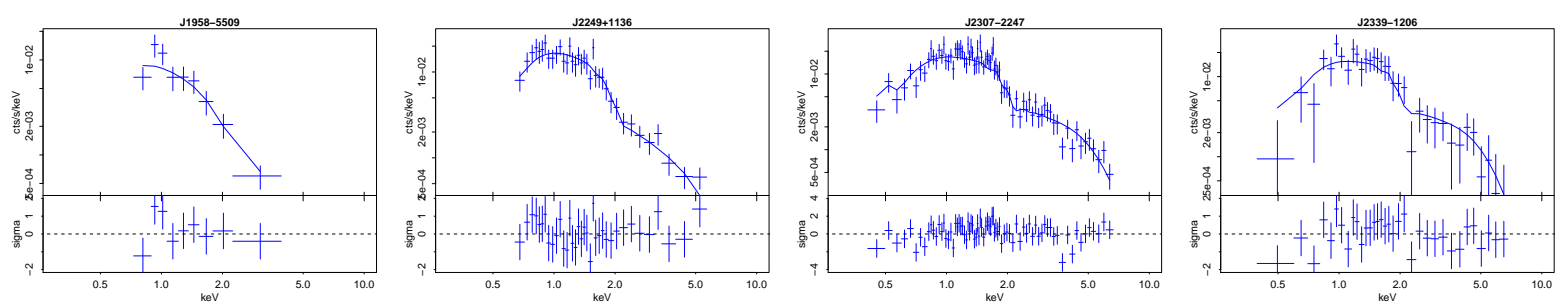

Fig. A.2. continued.
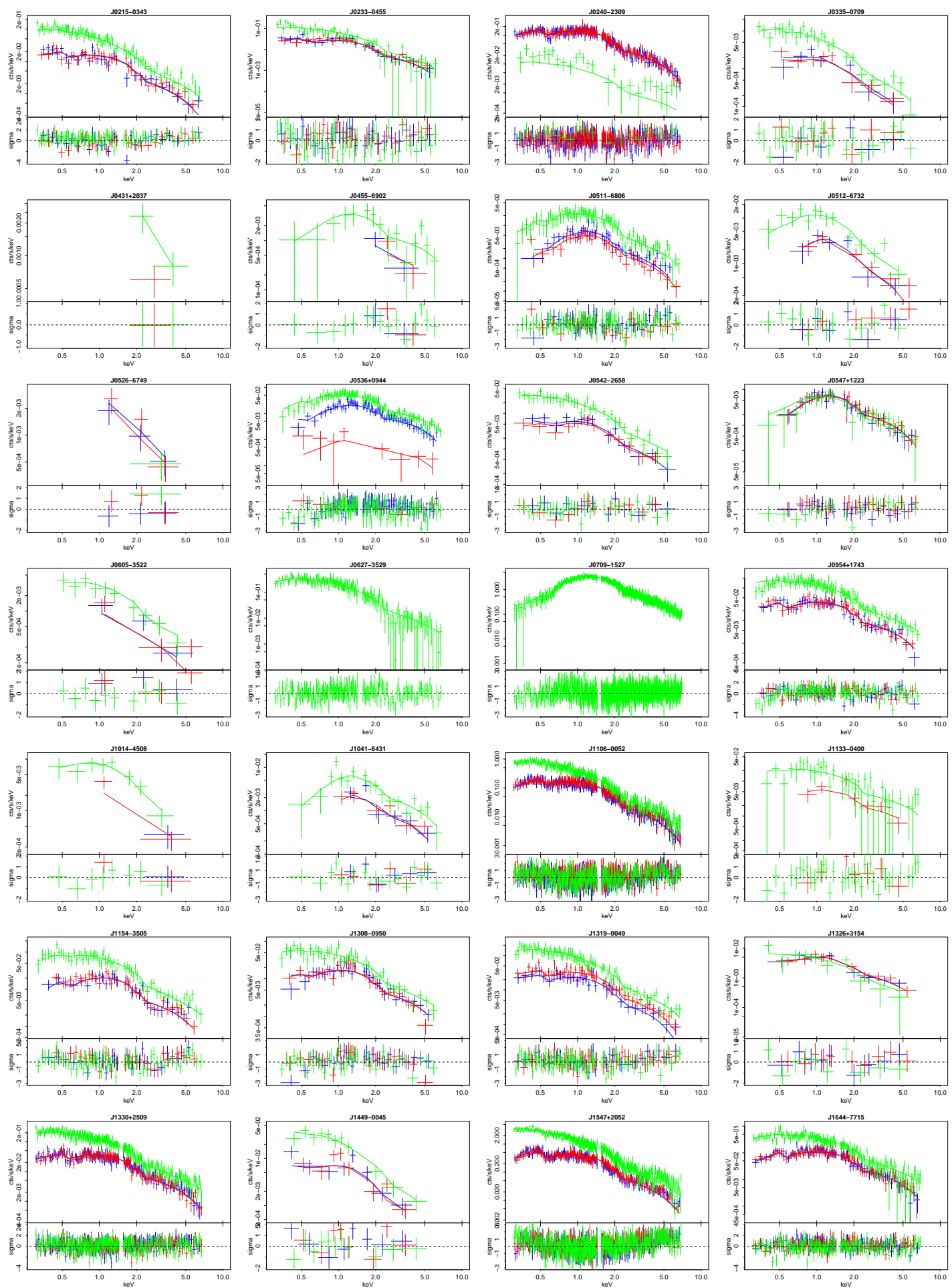

Fig. A.3. XMM-Newton-EPIC spectra with their best-fit power law models (upper panels) and residuals (lower panels). Lines and points in blue, red and green indicates models and data of MOS1, MOS2 and PN detectors, respectively. 
A. Paggi et al.: A new multiwavelength census of blazars
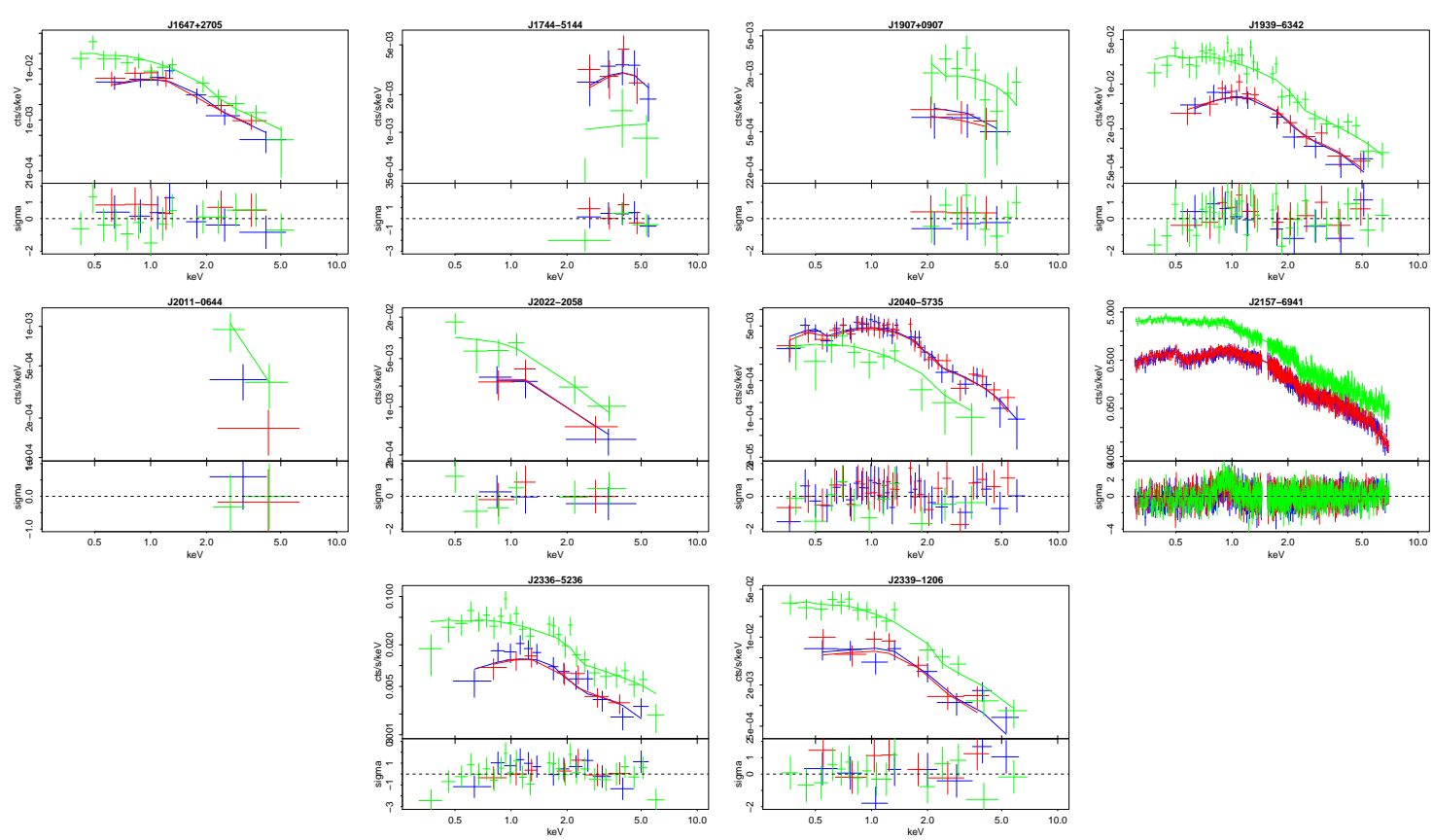

Fig. A.3. continued.
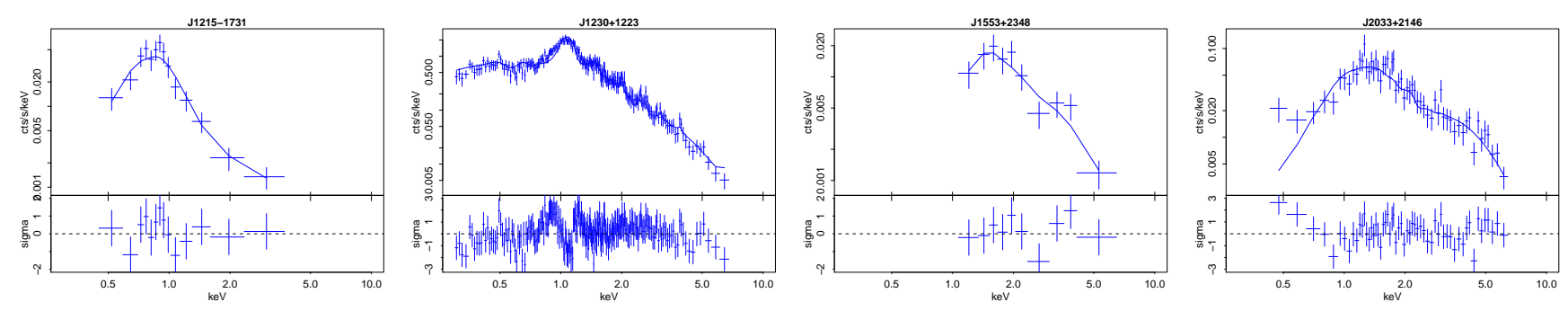

Fig. A.4. Swift-XRT spectra listed in Table 3 with their best-fit models (upper panels) and residuals (lower panels). 

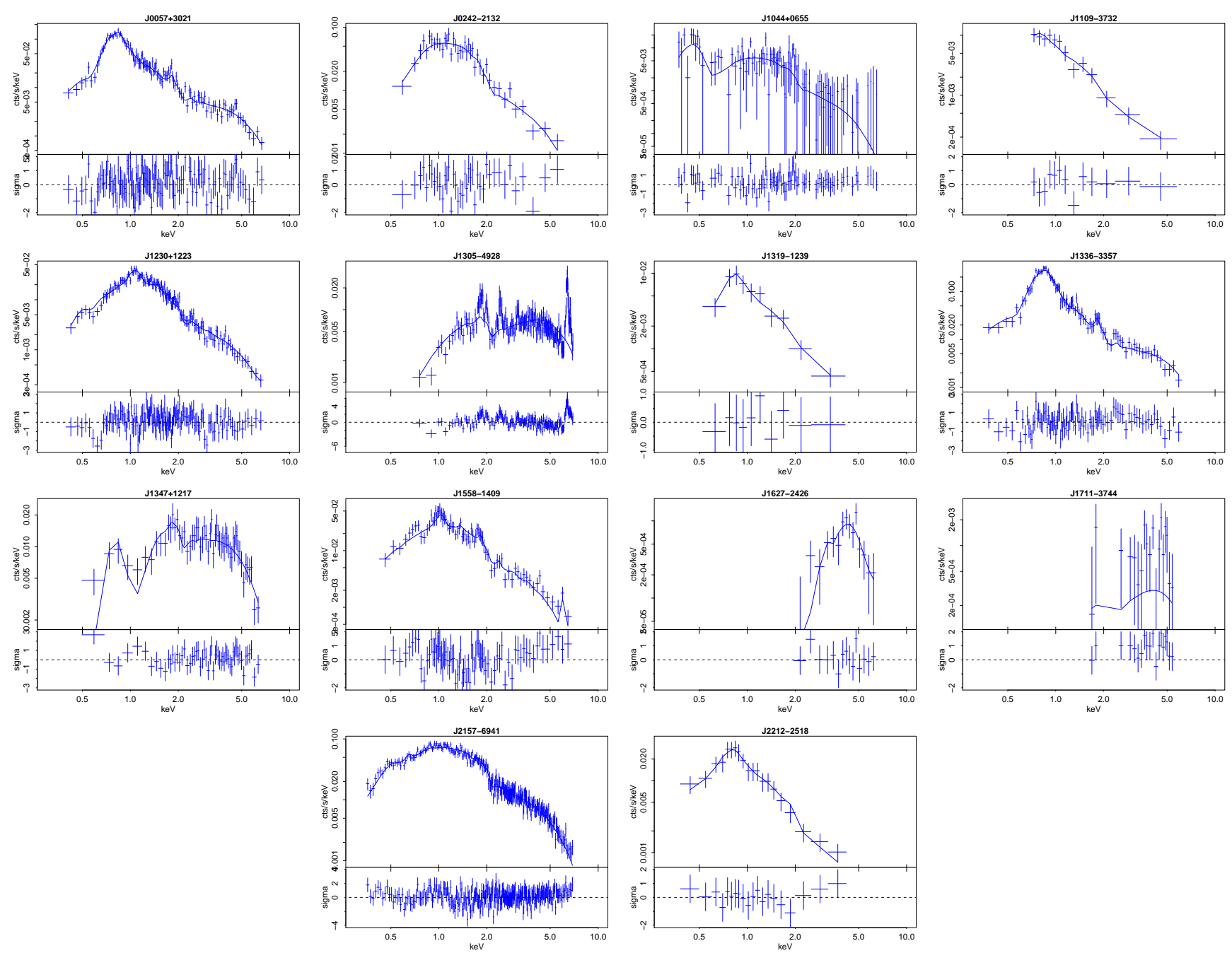

Fig. A.5. Chandra-ACIS spectra listed in Table 3 with their best-fit models (upper panels) and residuals (lower panels). 
A. Paggi et al.: A new multiwavelength census of blazars
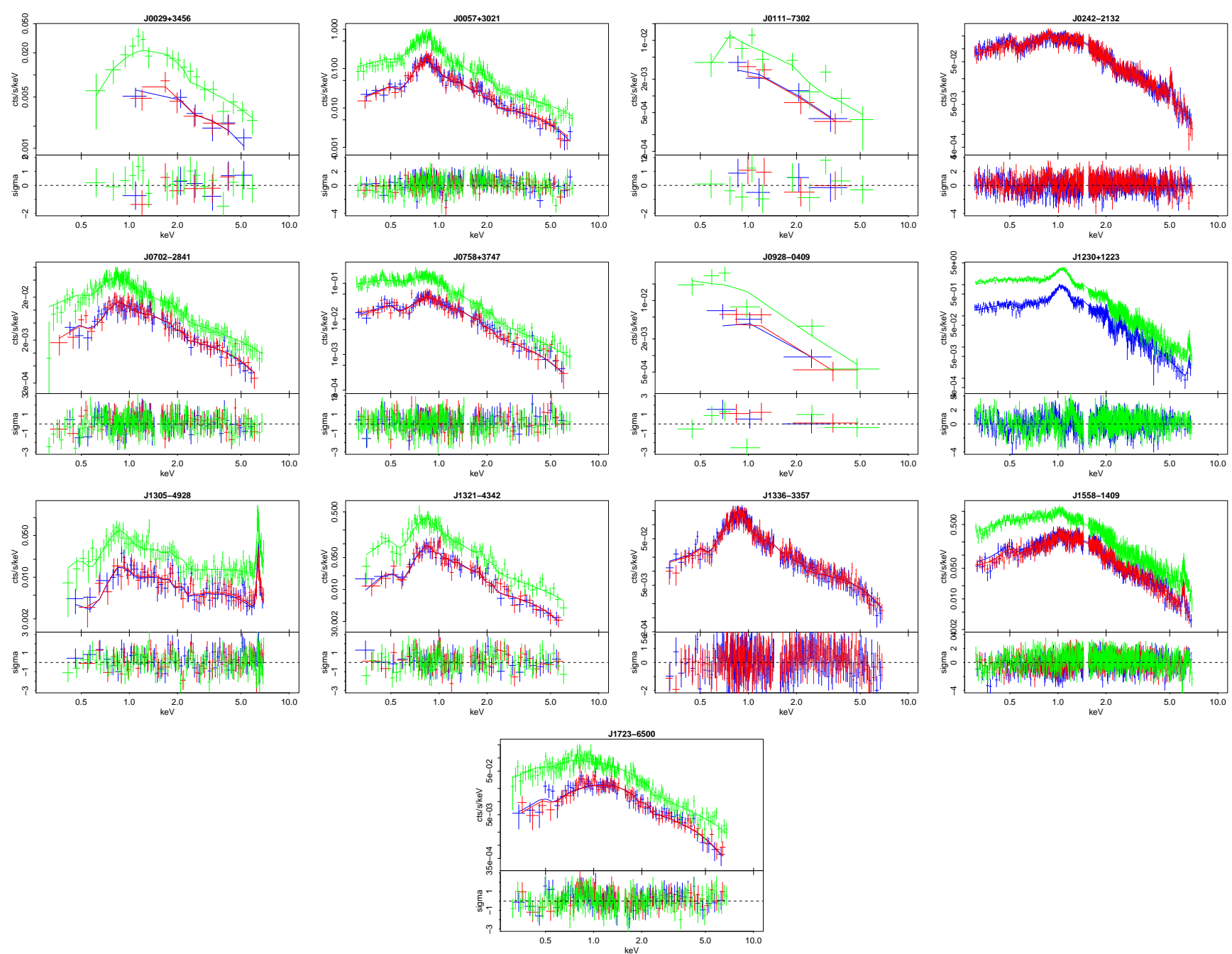

Fig. A.6. XMM-Newton-EPIC spectra listed in Table 3 with their best-fit models (upper panels) and residuals (lower panels). Lines and points in blue, red and green indicates models and data of MOS1, MOS2 and PN detectors, respectively. 


\section{Appendix B: Optical spectra}

In this appendix we collect the optical spectra collected for ABC sources as discussed in Sect. 4.1.3. In particular, in Fig. B.1 we present the SDSS DR12 spectra, while in Fig. B. 2 we present the LAMOST DR5 spectra.

J0024-0811

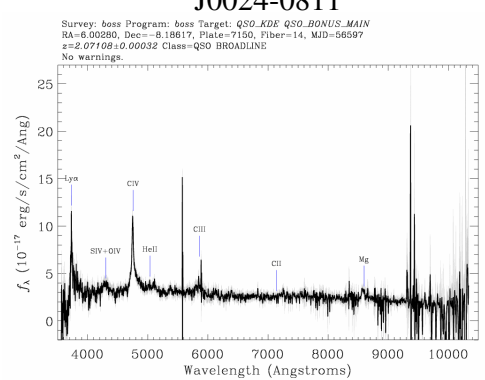

$\mathrm{J} 0029+3456$
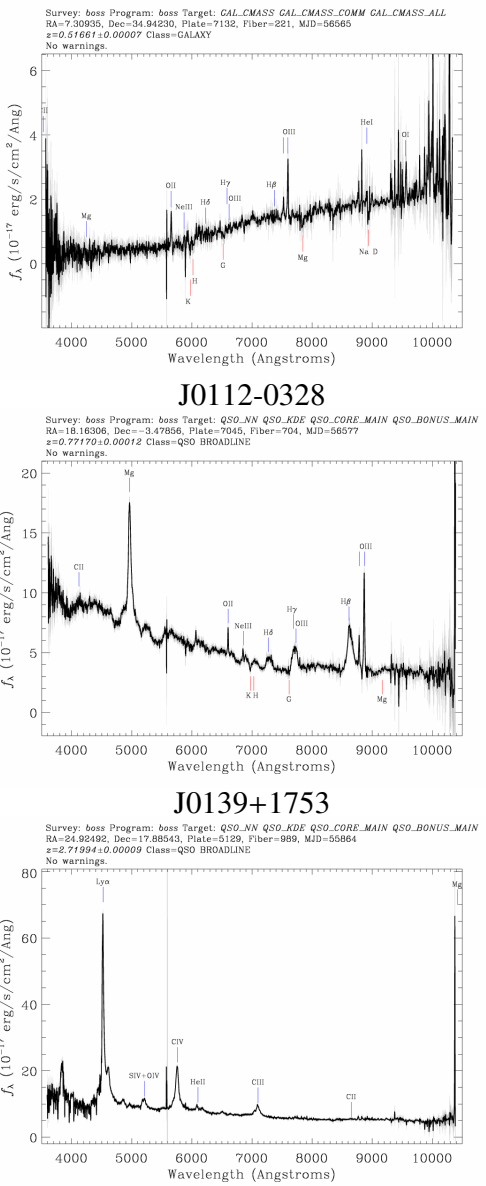

$\mathrm{J} 0158+1307$

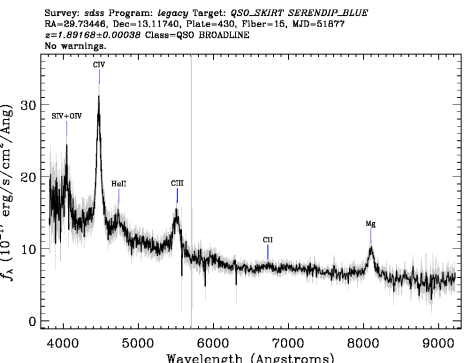

$\mathrm{J} 0028+2000$

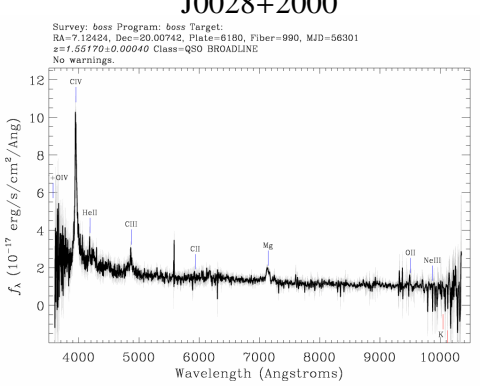

J0034-0054

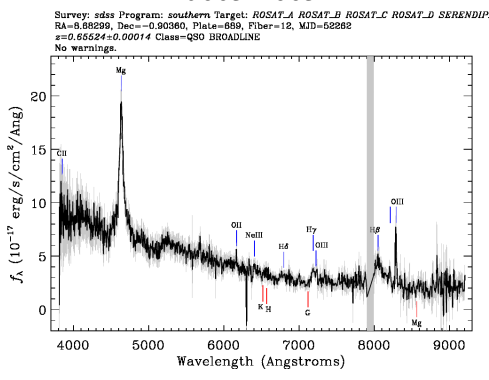

J0123-0923
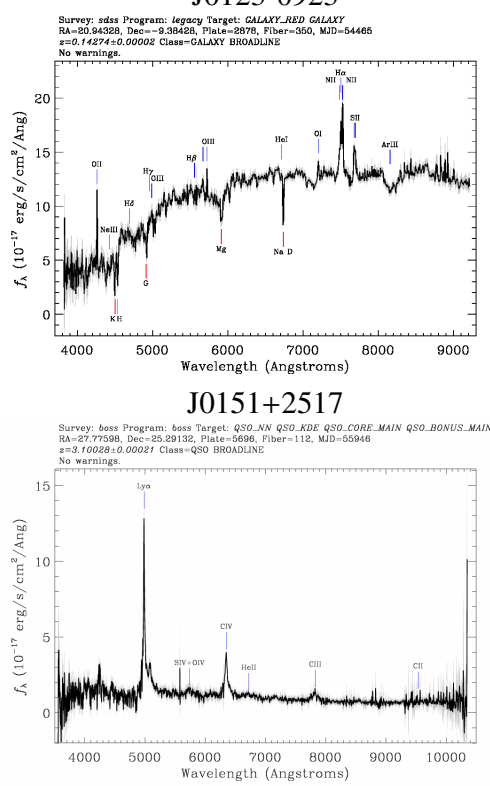

$\mathrm{J} 0200+0322$

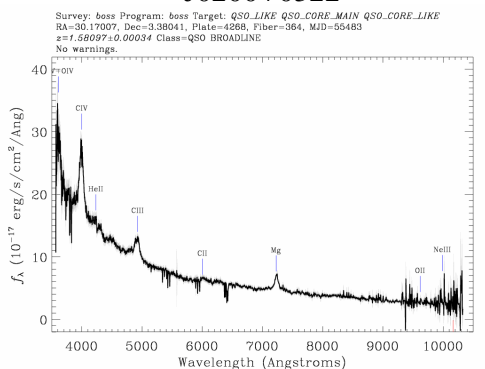

J0029-0113

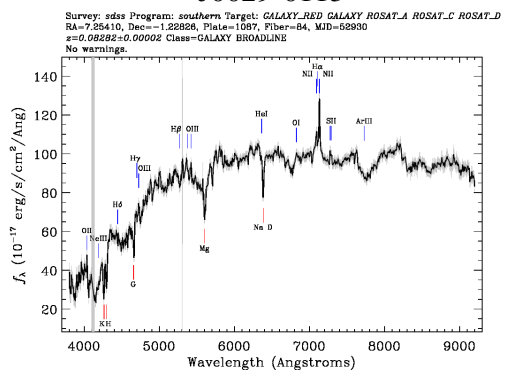

$\mathrm{J} 0107+1312$

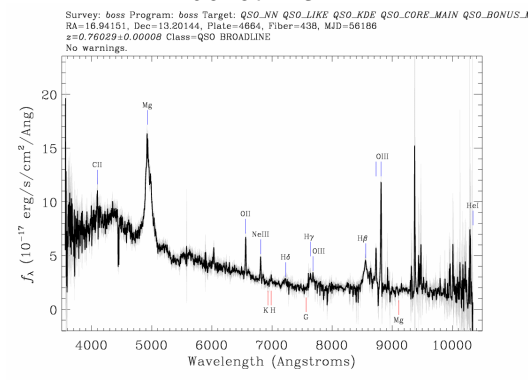

J0134-0931
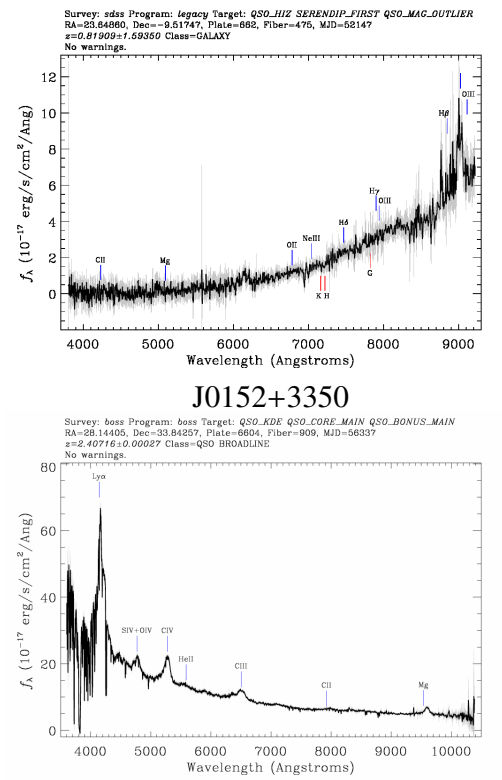

J0213-0719

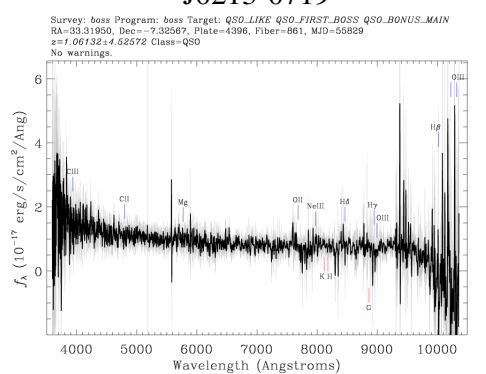

Fig. B.1. SDSS DR12 optical spectra for the $98 \mathrm{ABC}$ sources discussed in Sect. 4.1.3. 
A. Paggi et al.: A new multiwavelength census of blazars

J0215-0343

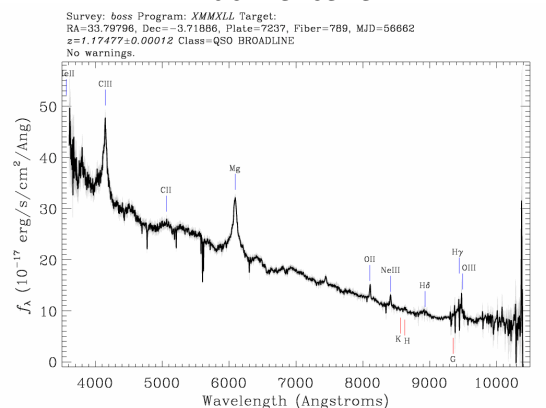

J0743+3941

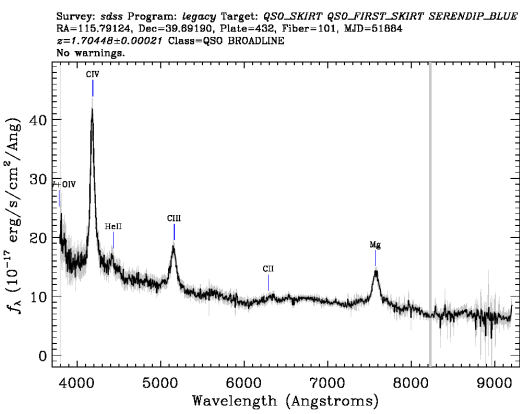

$\mathrm{J} 0758+3747$

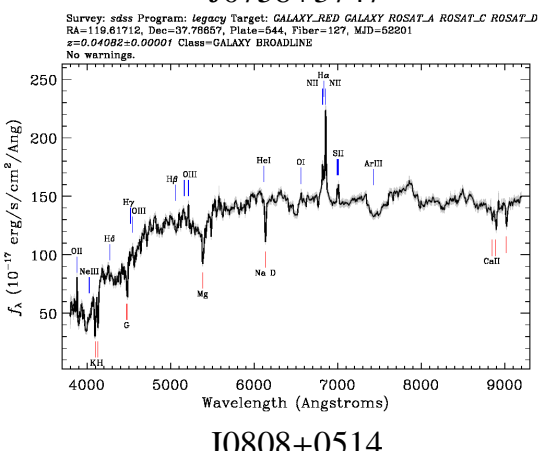

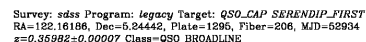

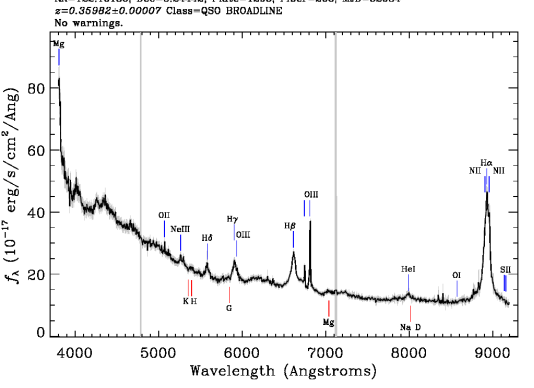

J0832+1832

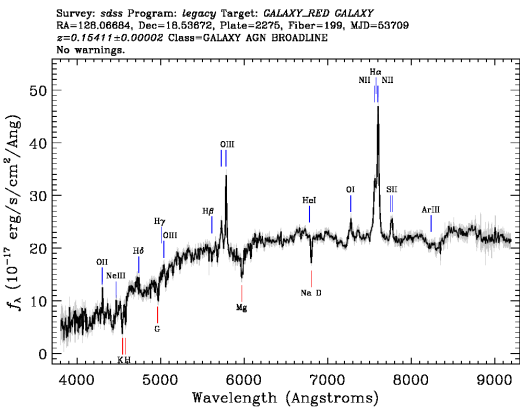

J0233-0455

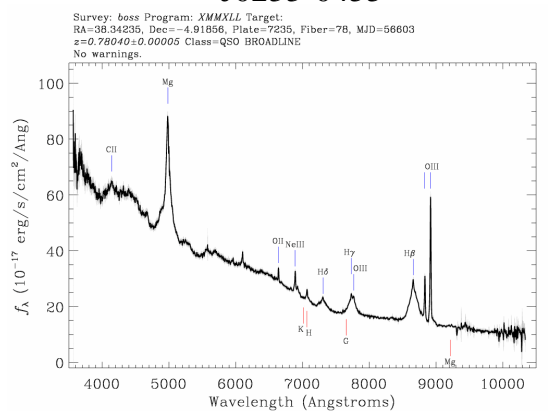

J0745+3142

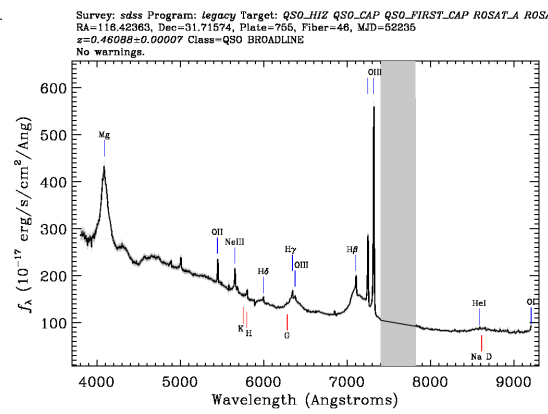

$\mathrm{J} 0802+2509$
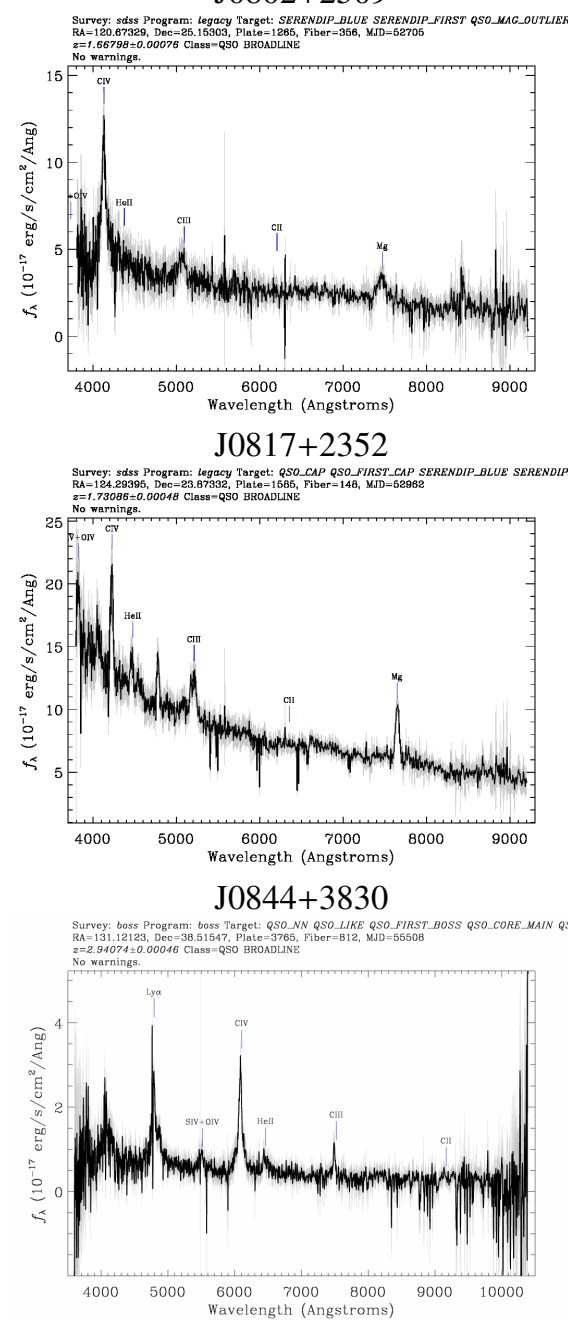

J0408-0529

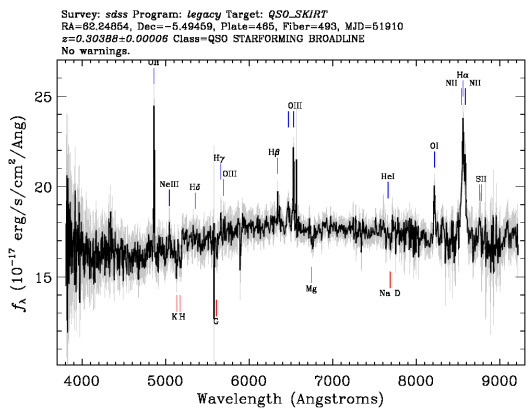

J0758+1136

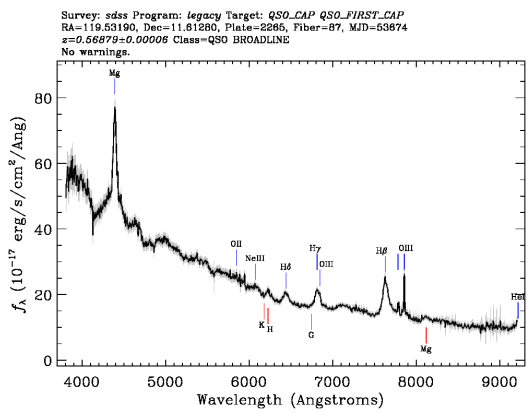

J0807+1352

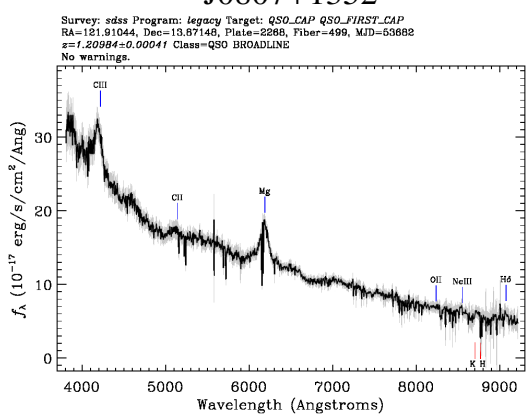

J0821+2857
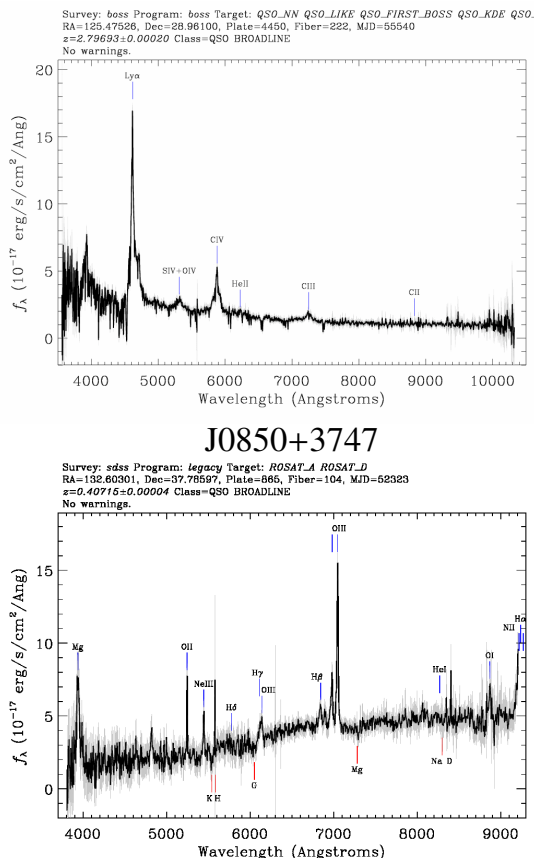

Fig. B.1. continued. 
$\mathrm{J} 0853+0654$

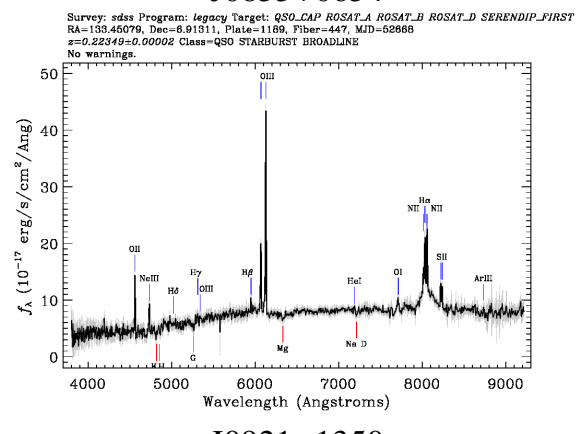

J0921+1350

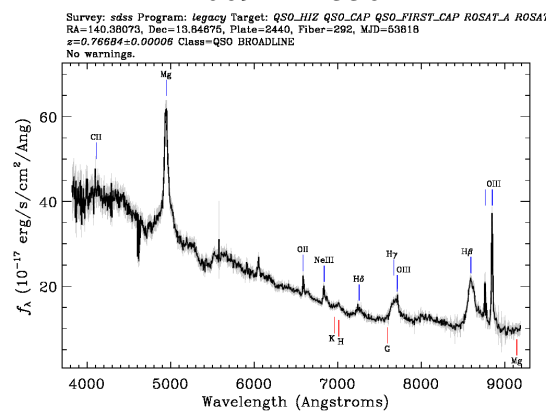

J0945+3534
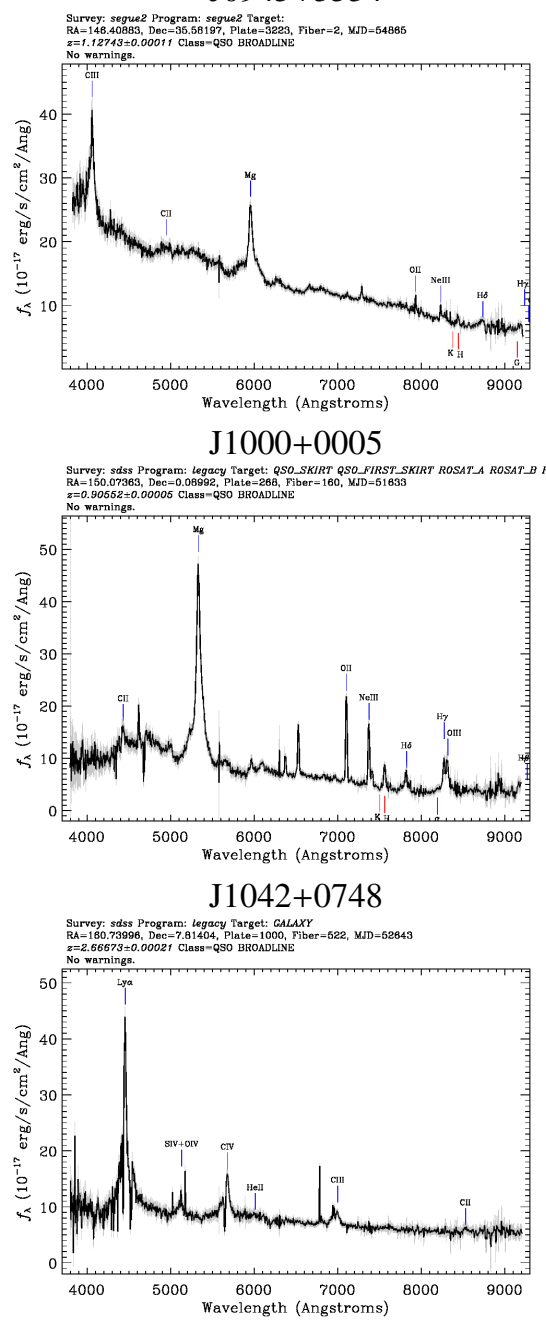

J0912+2205

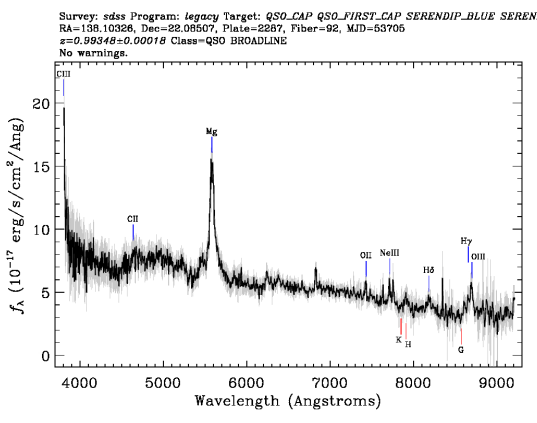

J0931+1414

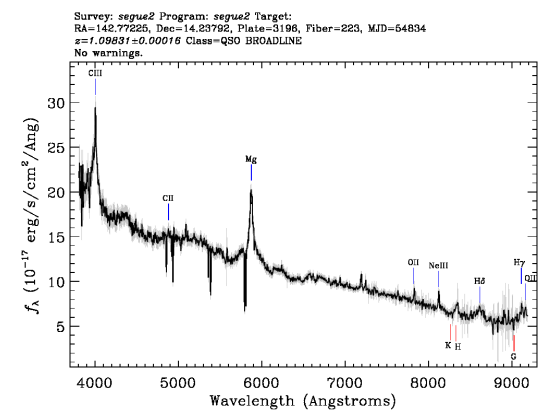

J0945-0153

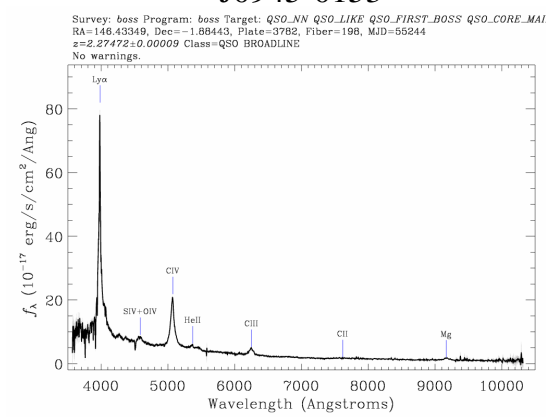

J1024-0052
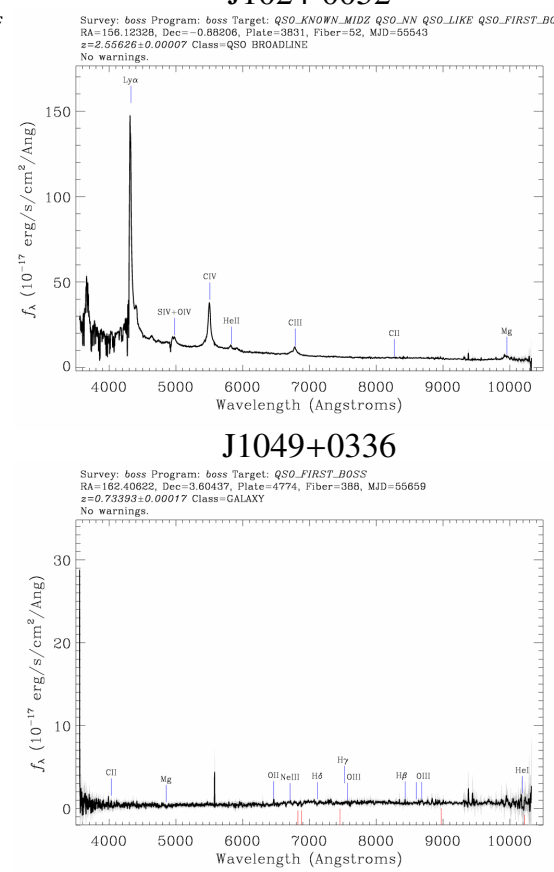

J0914+3512
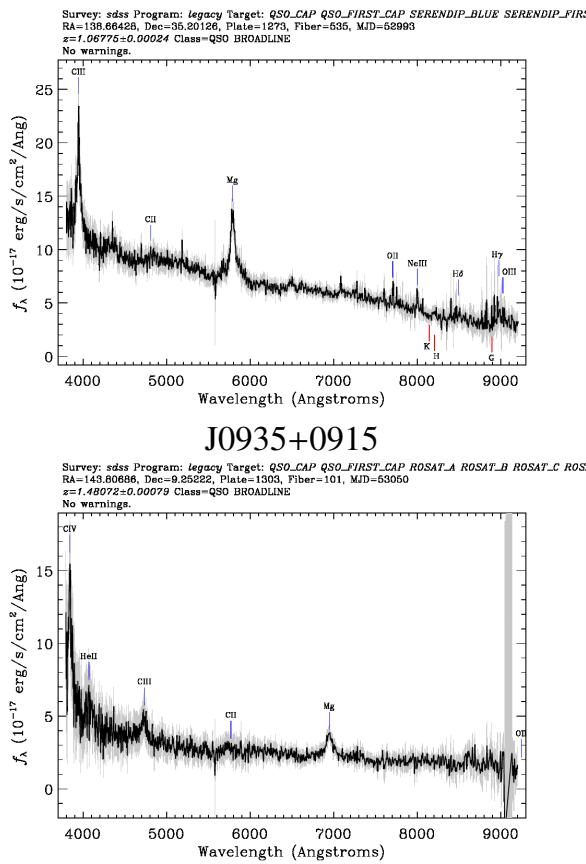

J0954+1743

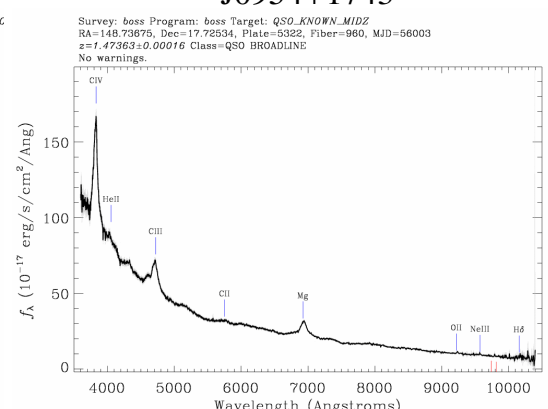

J1029+0519

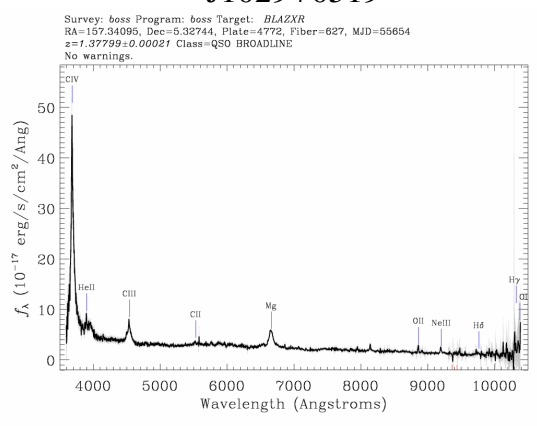

$\mathrm{J} 1103+1158$

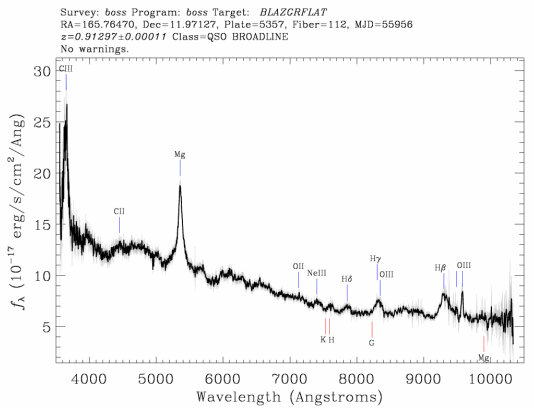

Fig. B.1. continued. 
J1106-0052

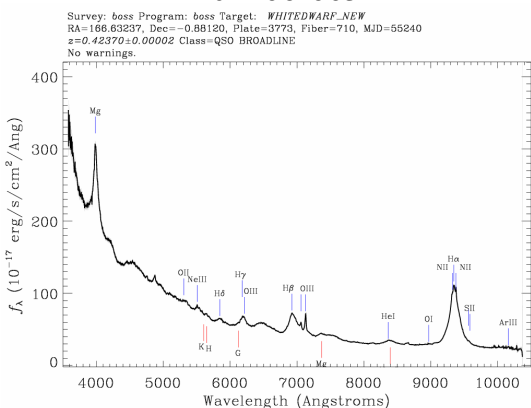

J1125+2005

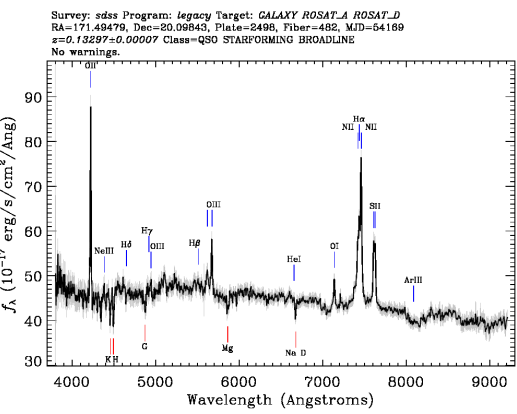

J1148-0046

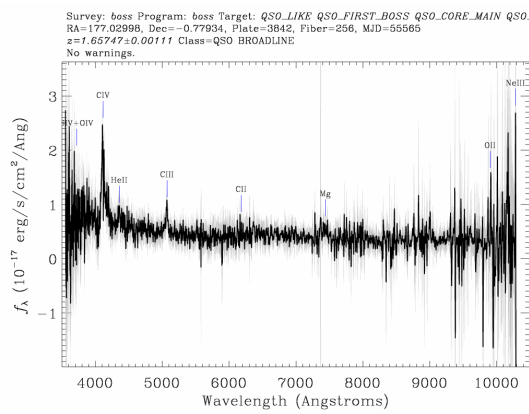

$\mathrm{J} 1211+1820$

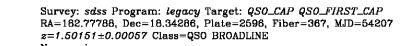

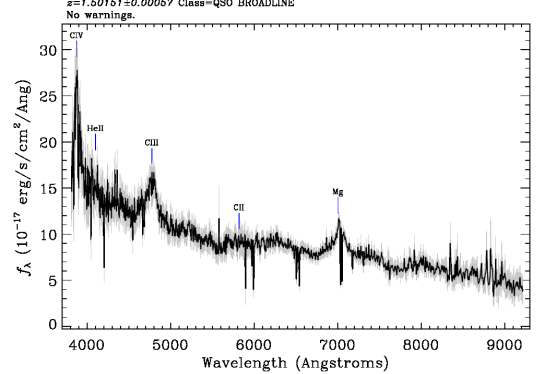

J1218+1105

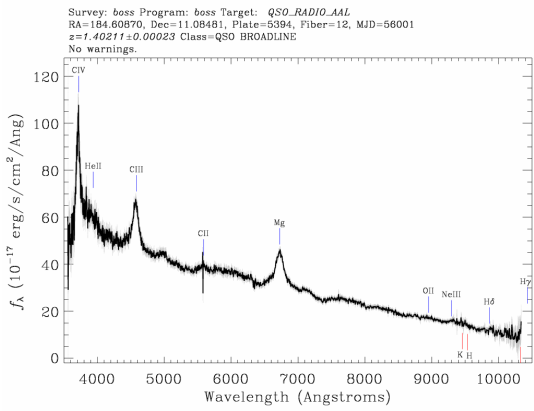

$\mathrm{J} 1107+1628$

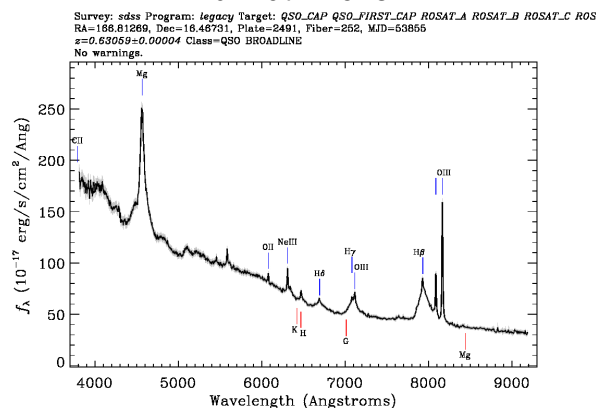

J1130+0846

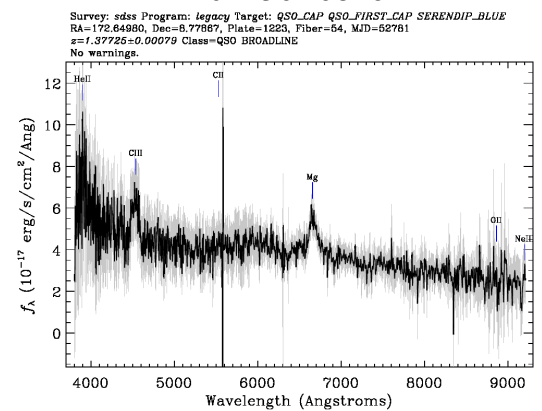

$\mathrm{J} 1157+1638$

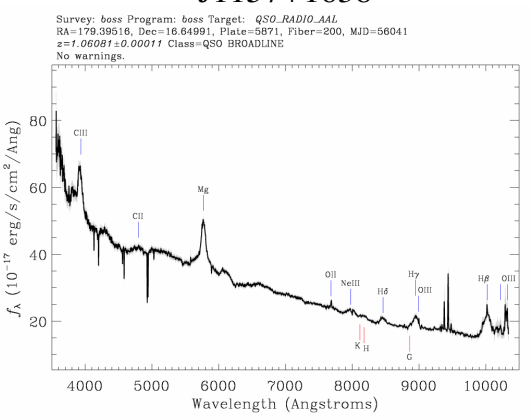

J1214+3309

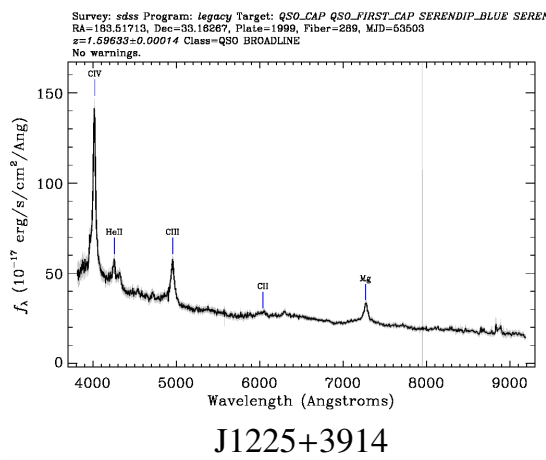

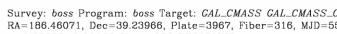

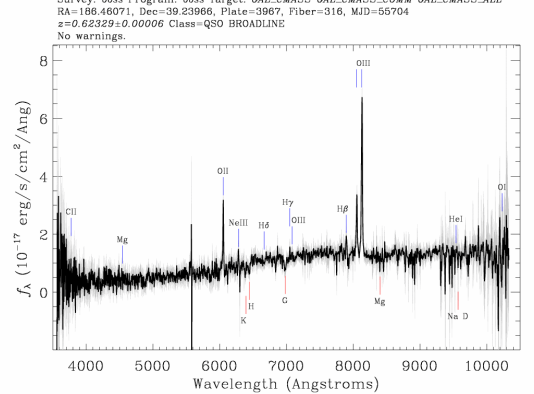

J1124+2307

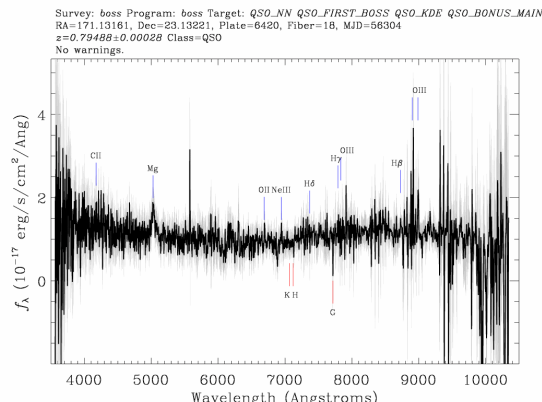

J1130+3031

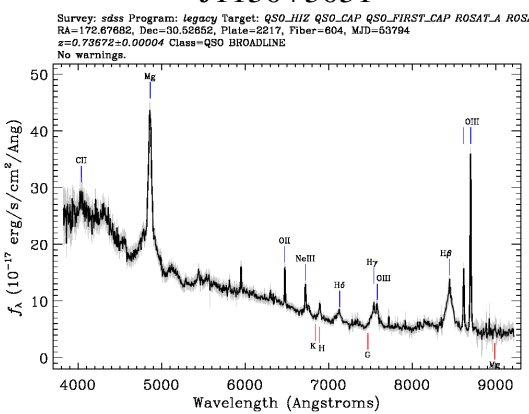

J1206+3941

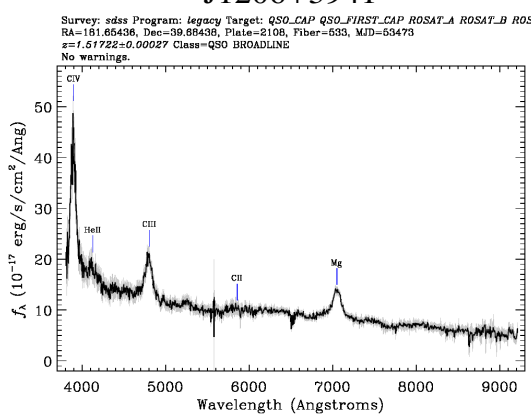

$\mathrm{J} 1215+3151$

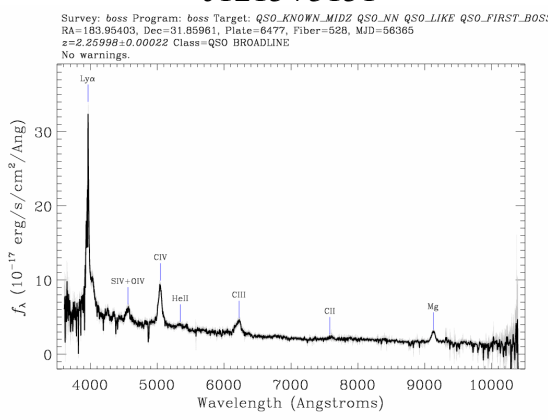

J1232+1809

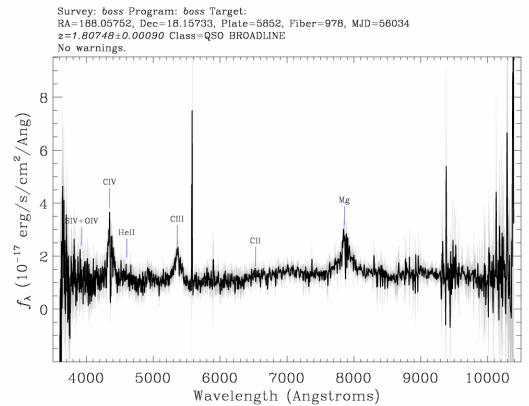

Fig. B.1. continued. 
$\mathrm{J} 1237+0755$

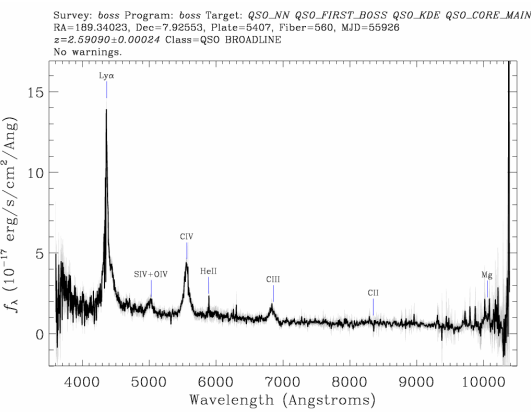

$\mathrm{J} 1243+1622$

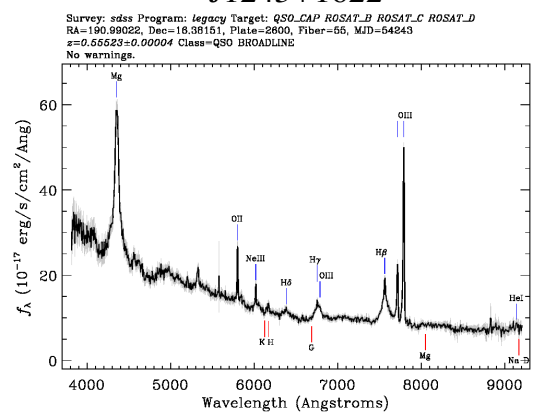

$\mathrm{J} 1300+0828$

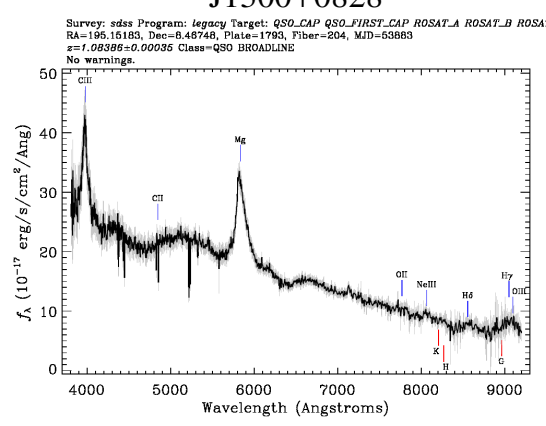

$\mathrm{J} 1330+2509$

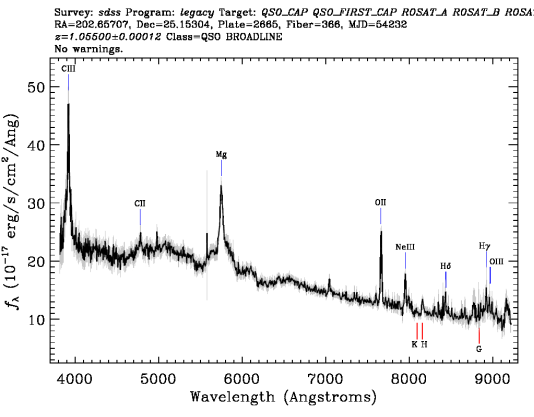

J1419+0628

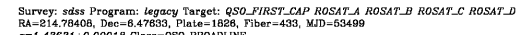

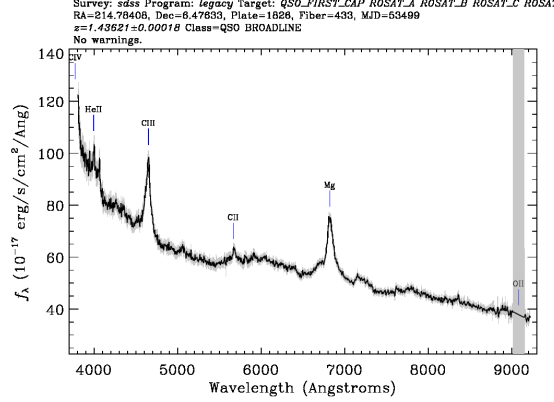

$\mathrm{J} 1237+1924$

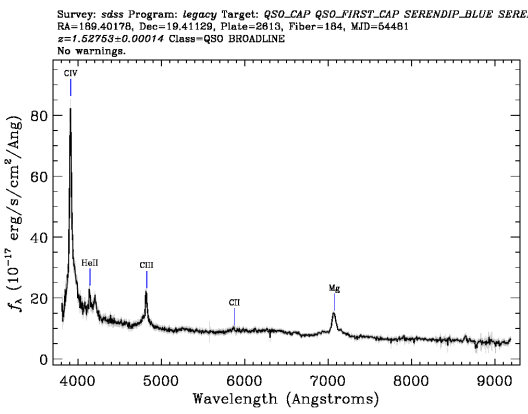

$\mathrm{J} 1248+2022$

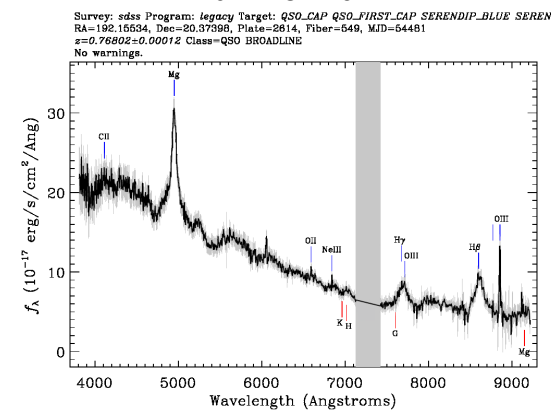

$\mathrm{J} 1315+1220$

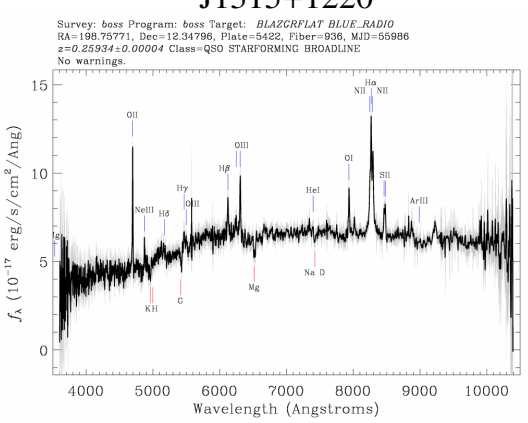

$\mathrm{J} 1331+3030$

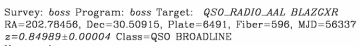
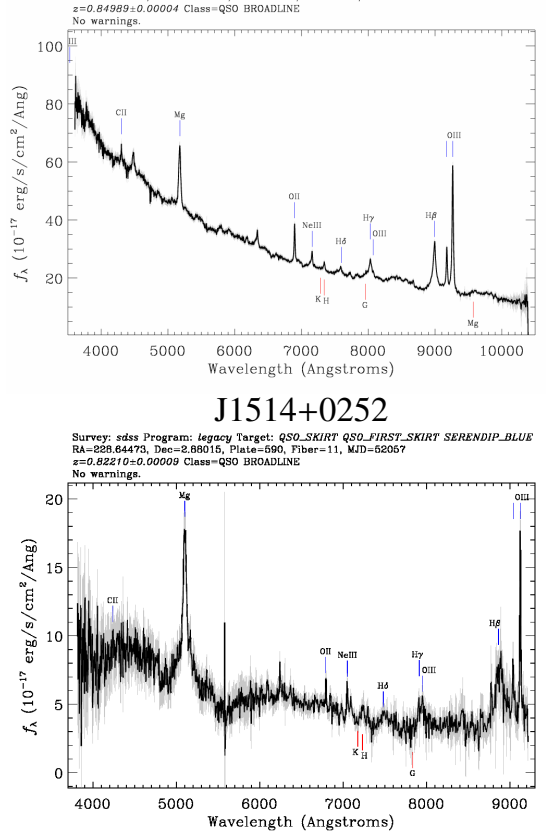

$\mathrm{J} 1242+0941$

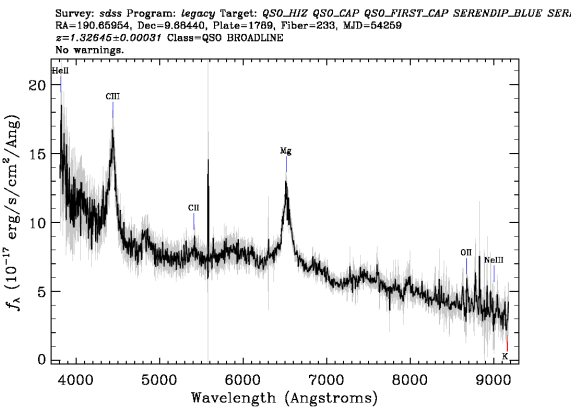

$\mathrm{J} 1250+1343$

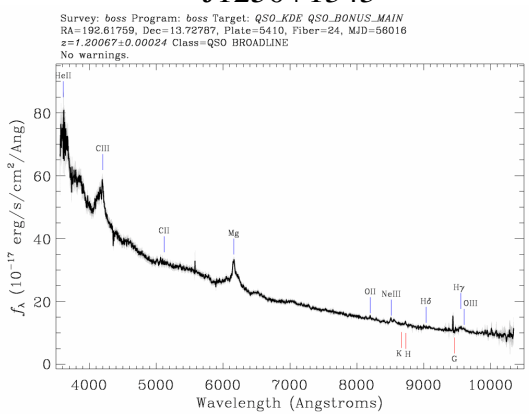

J1319-0049

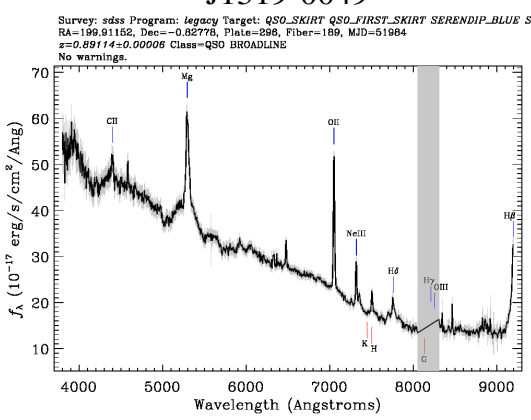

$\mathrm{J} 1332+0200$
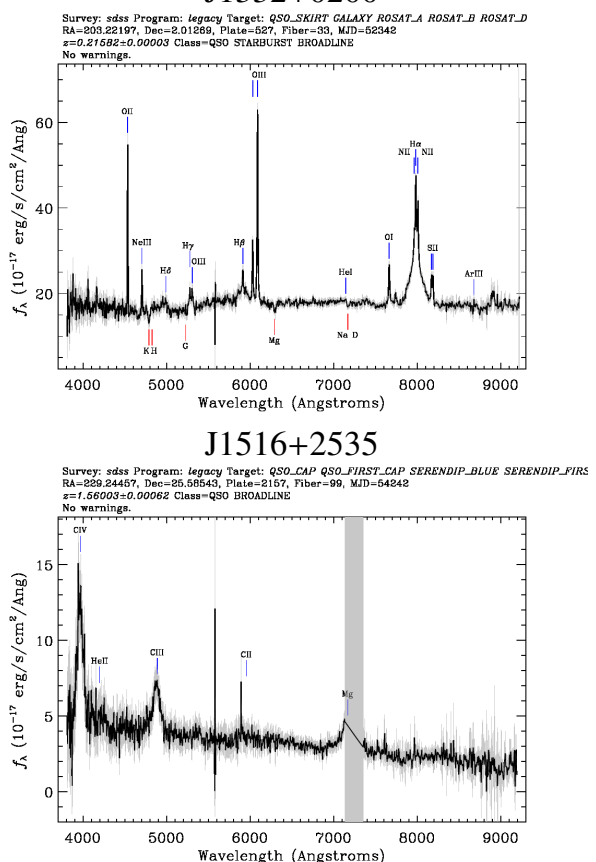

Fig. B.1. continued. 
$\mathrm{J} 1521+0517$

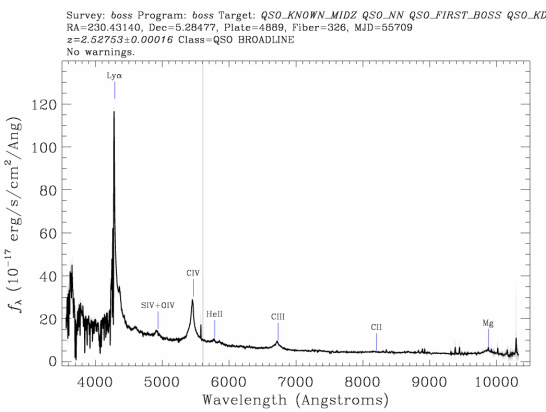

$\mathrm{J} 1537+2300$

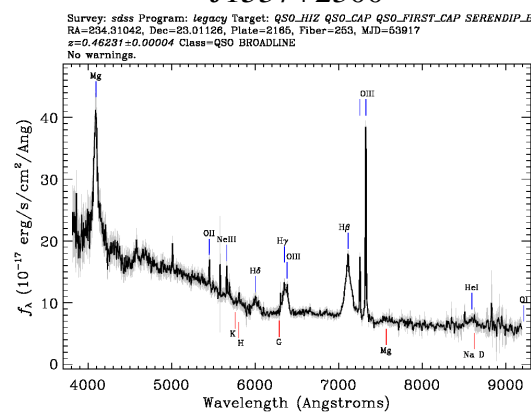

$\mathrm{J} 1545+2006$

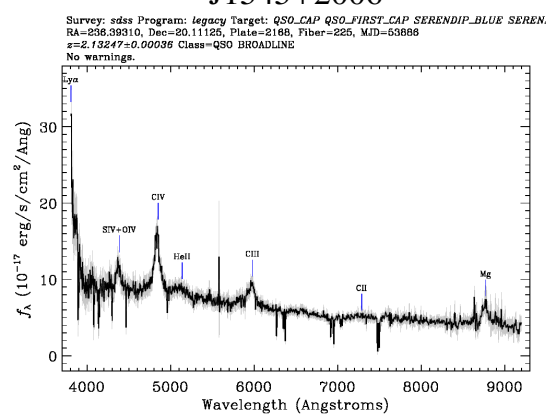

J1602+2418

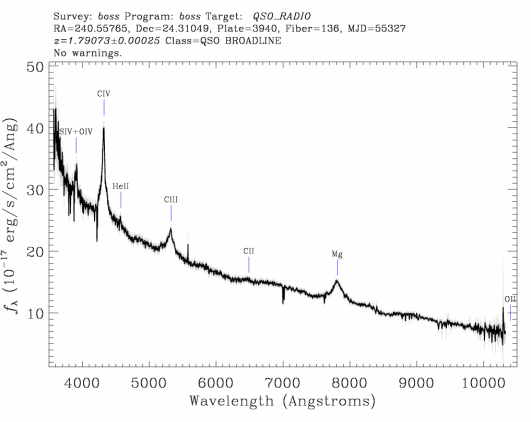

J1647+2705

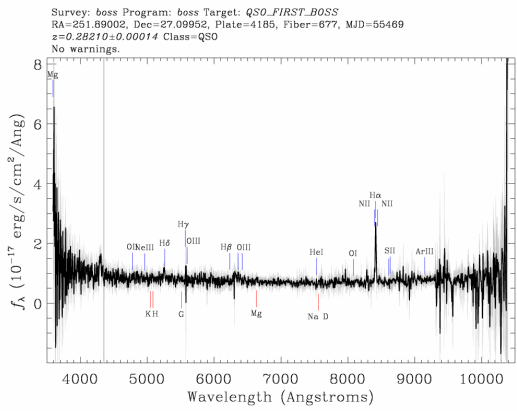

$\mathrm{J} 1521+1756$

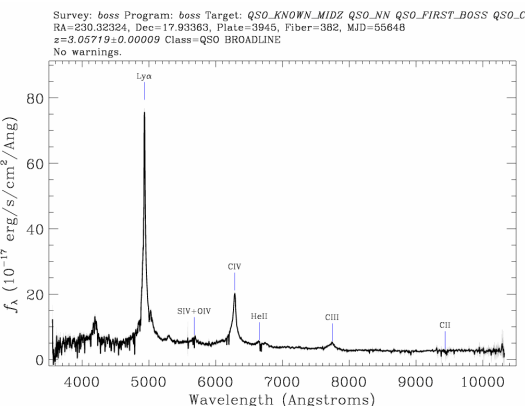

$\mathrm{J} 1539+0534$

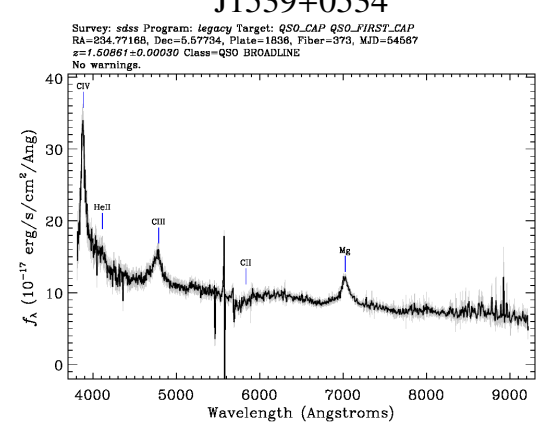

$\mathrm{J} 1547+2052$

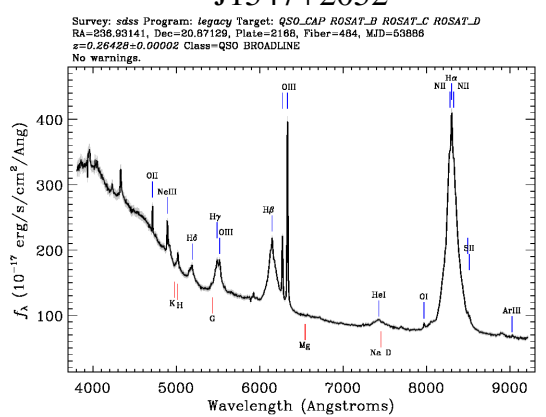

J1606+1814
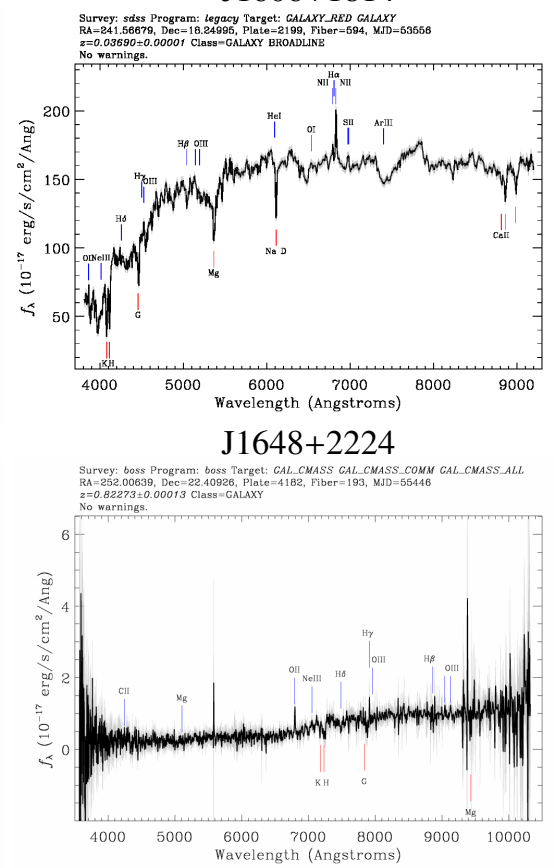

$\mathrm{J} 1532+2344$

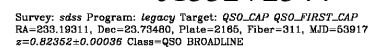

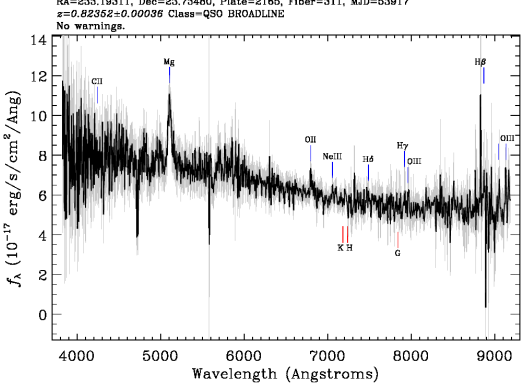

J1544+0407

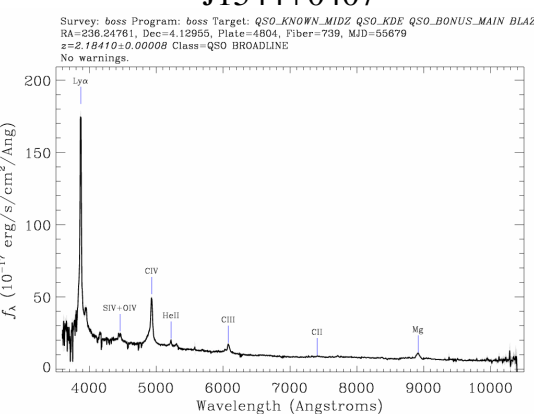

$\mathrm{J} 1553+2348$

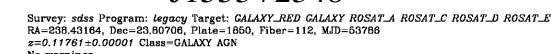

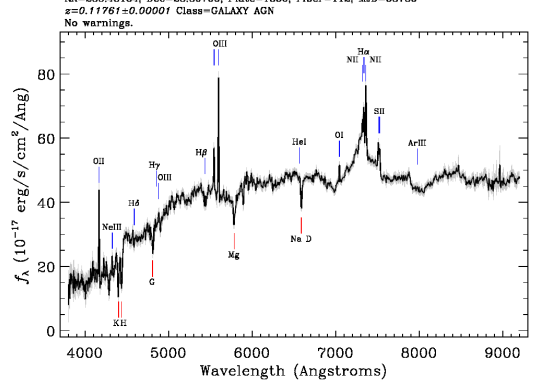

J1634+3203

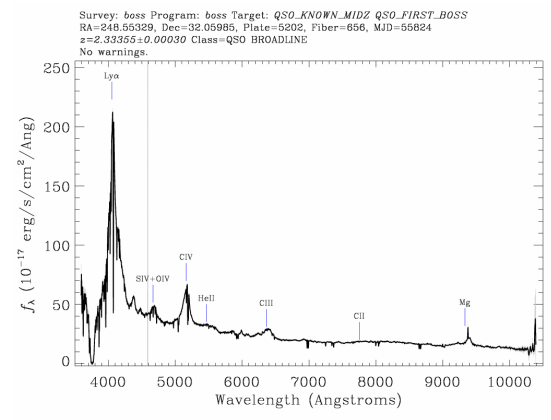

$\mathrm{J} 1659+2629$

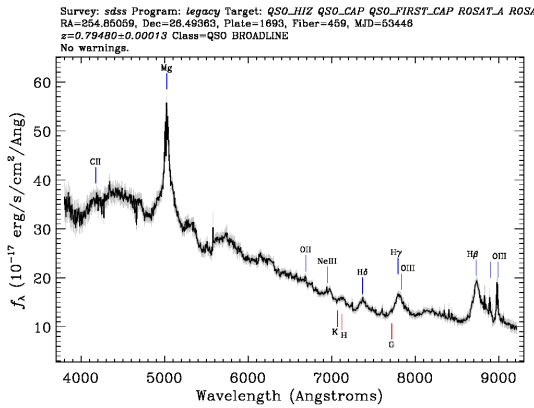

Fig. B.1. continued. 
J2116+0536

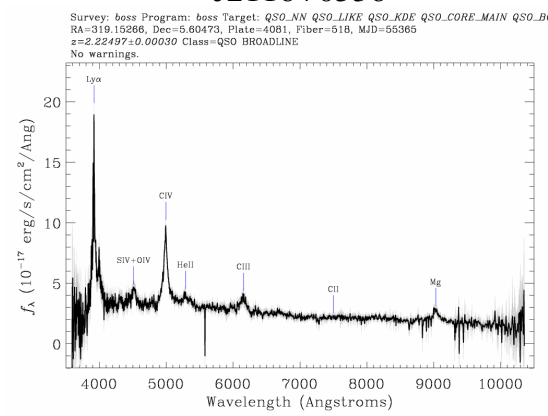

J2240-0836

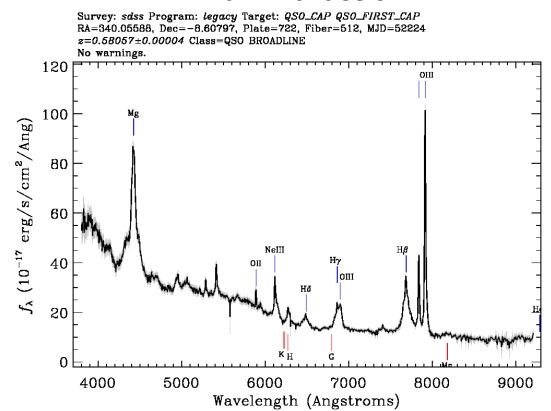

J2136-0643

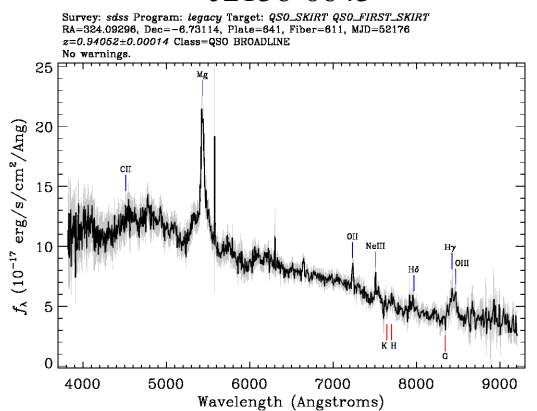

J2312-0109

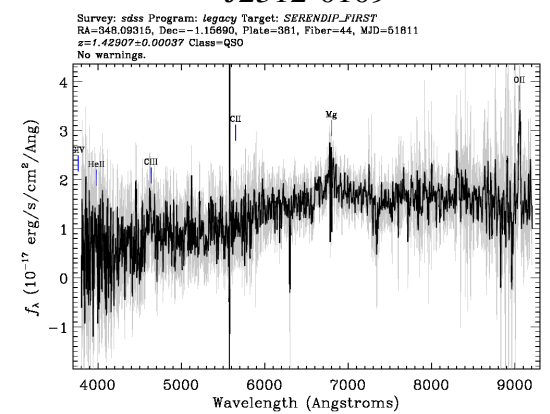

$\mathrm{J} 2153+1241$

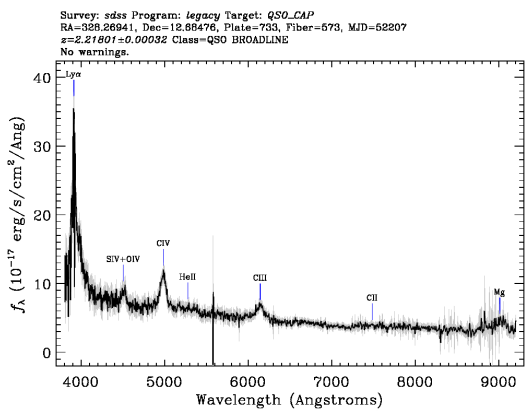

J2340-0053

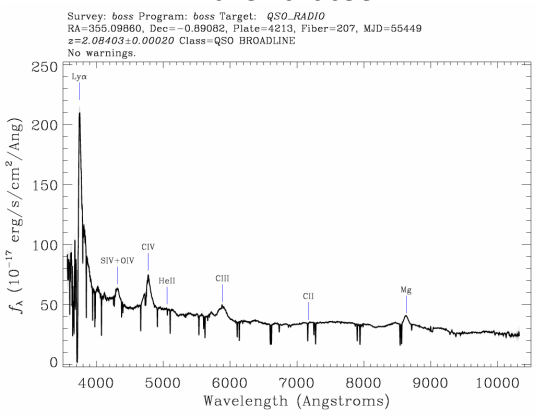

J2346+0705

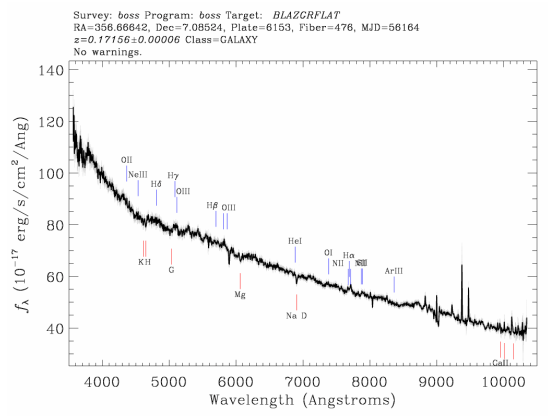

J2359-0031

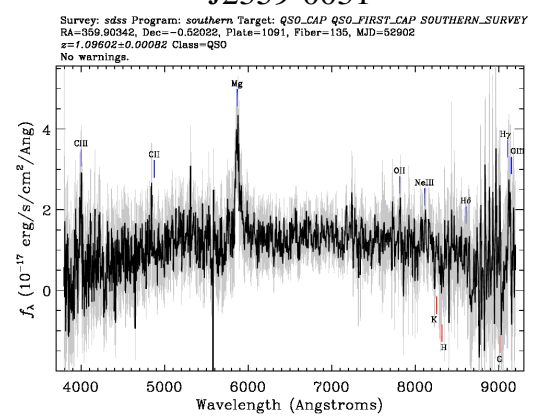

Fig. B.1. continued. 
A. Paggi et al.: A new multiwavelength census of blazars
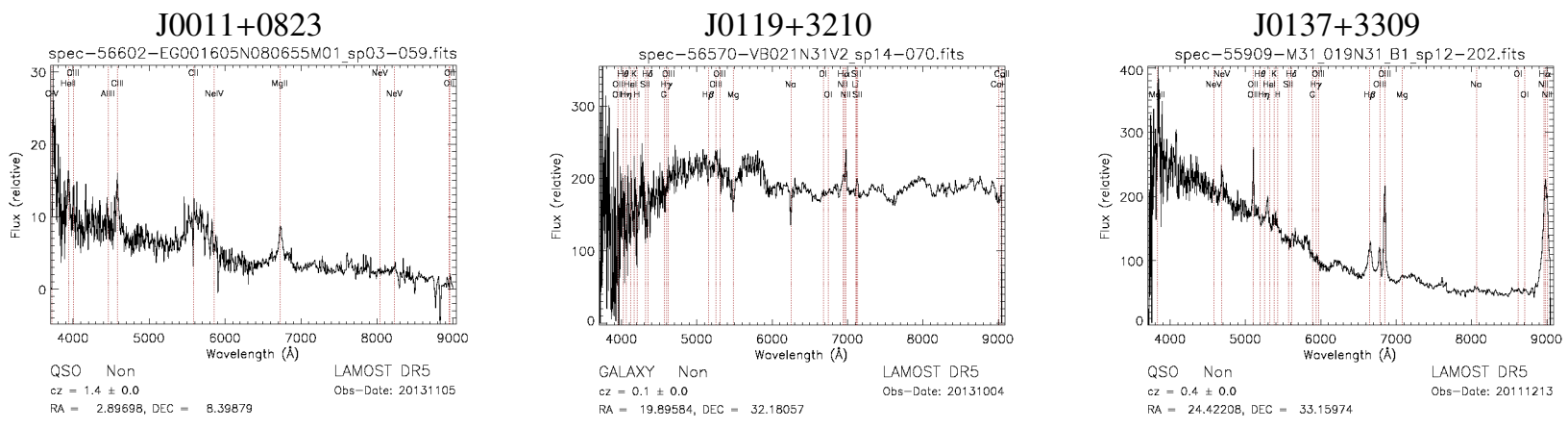

$\mathrm{J} 0152+3350$

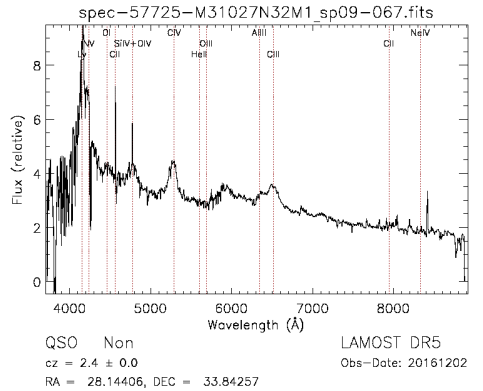

J0156+3914

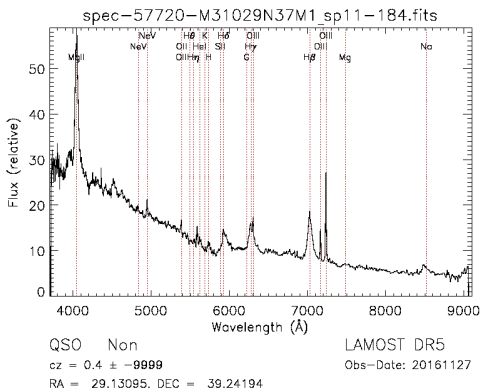

J0204+3649

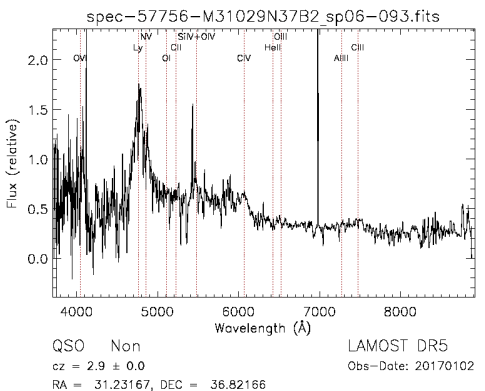

J0954+1743

J0945+3534

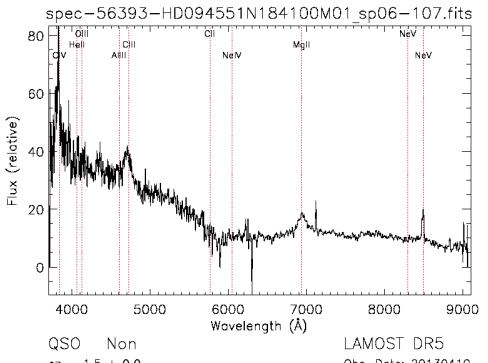

$\begin{array}{ll}Q S O & \text { Non } \\ c z=1.5 \pm 0.0\end{array}$

$\mathrm{J} 1000+0005$

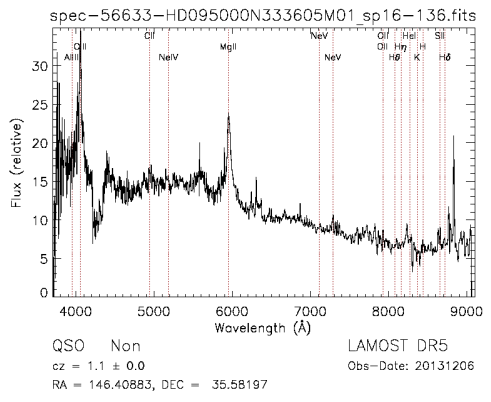

J0958+3224

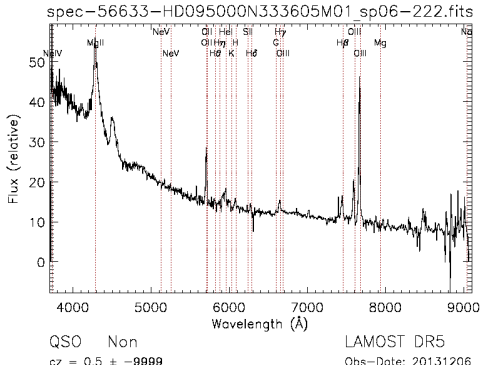

J1024-0052

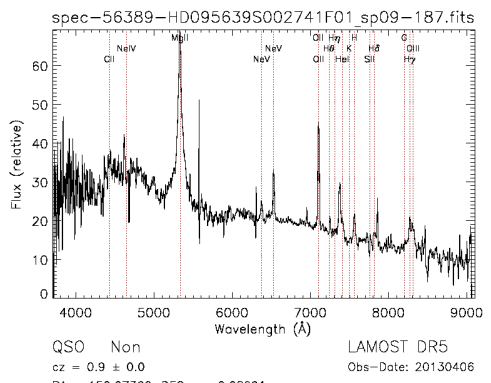

PA $=15007356, D E C=0.0899$

$\mathrm{J} 1101+0215$

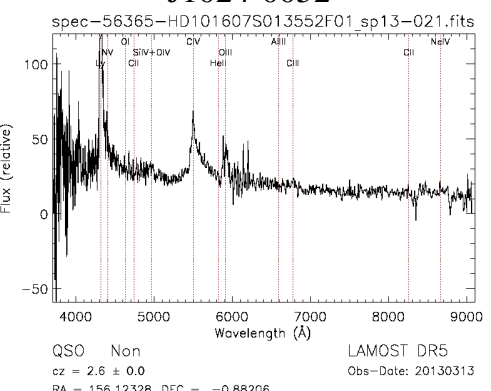

$\mathrm{J} 1107+1628$
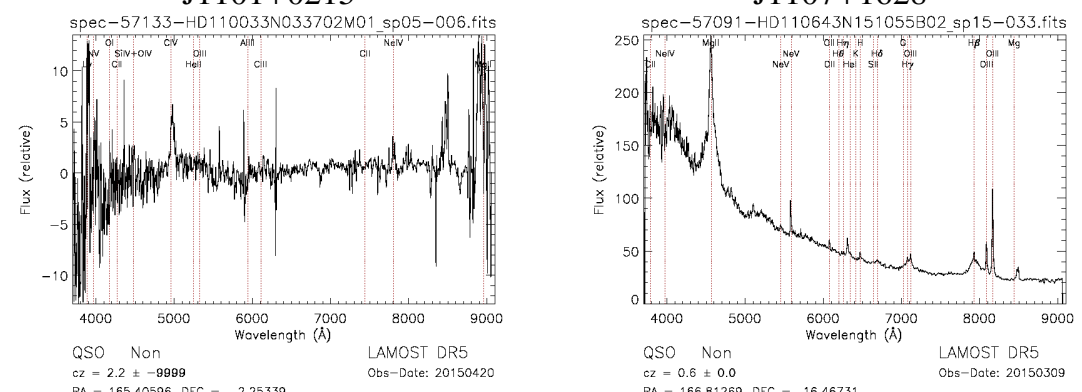

$c z=0.5 \pm-999$

J1050-0318

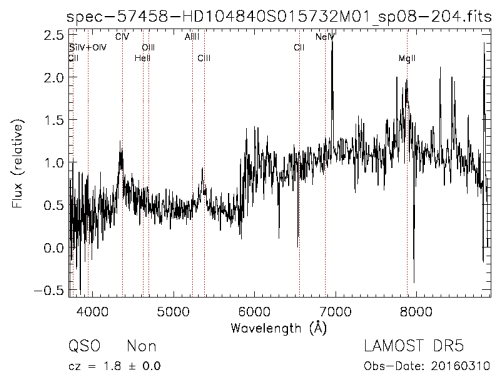

$\mathrm{J} 1130+3031$

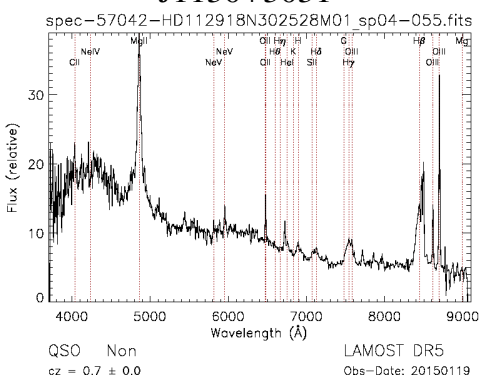

Fig. B.2. LAMOST DR5 optical spectra for the $28 \mathrm{ABC}$ sources discussed in Sect. 4.1.3. 


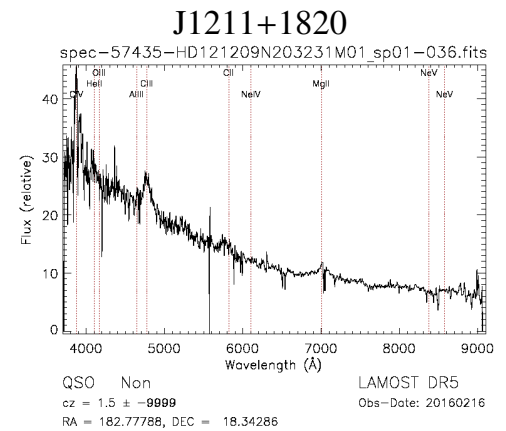

$\mathrm{J} 1242+0941$

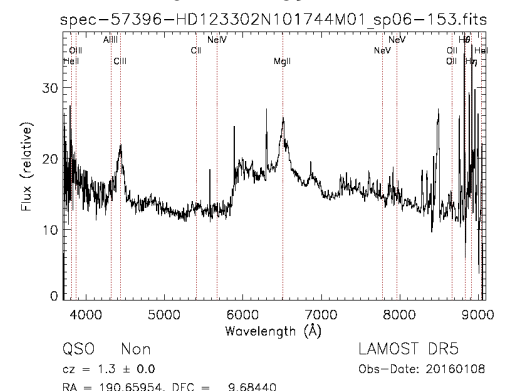

$\mathbf{J} 1331+3030$

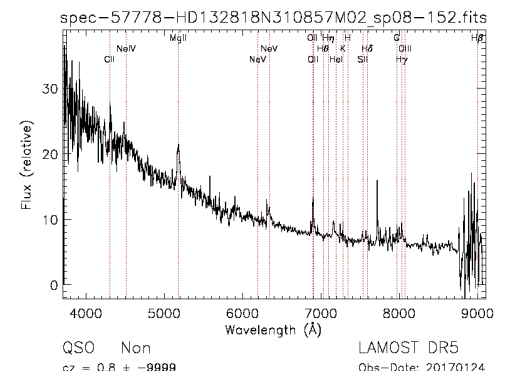

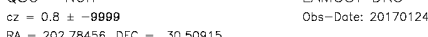

$\mathrm{J} 2149+0756$

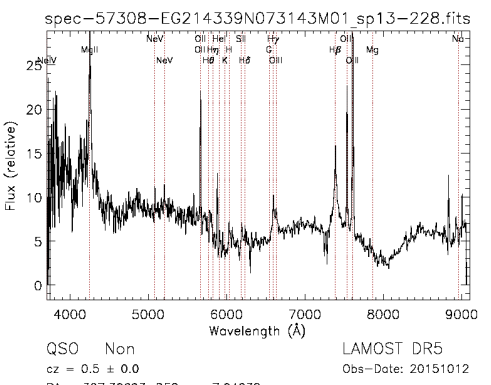

$\mathrm{J} 1226+1352$

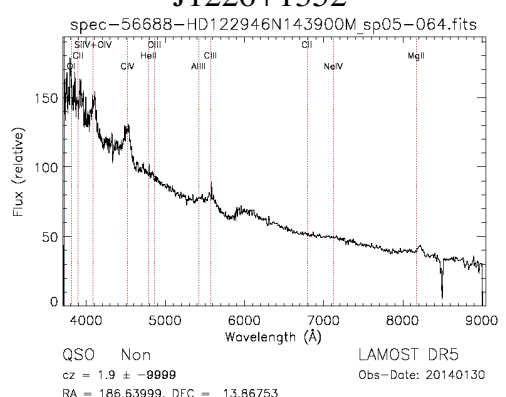

$\mathrm{J} 1243+1622$

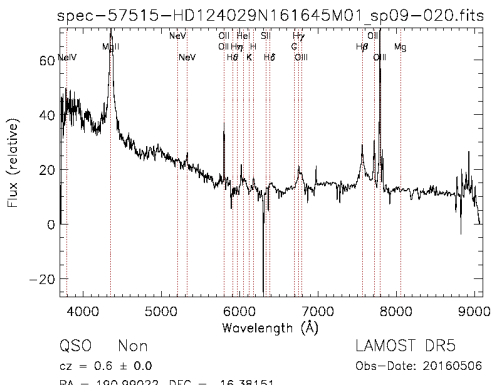

J1419+0628

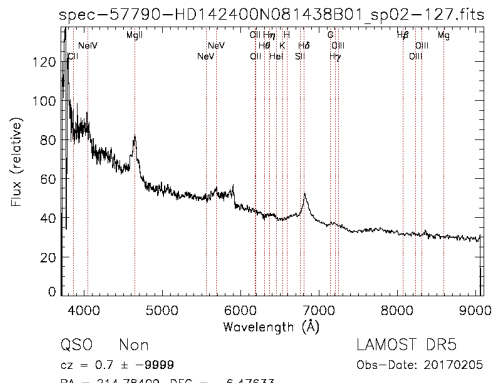

$\mathrm{J} 2221+1117$

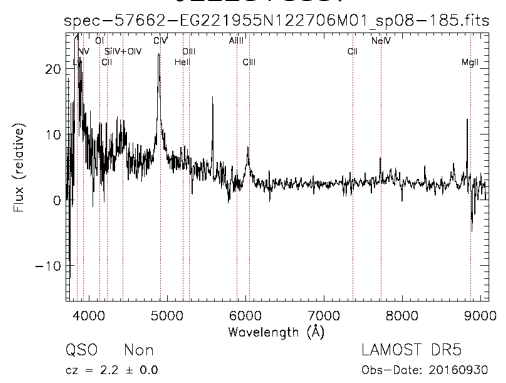

J1237+1924

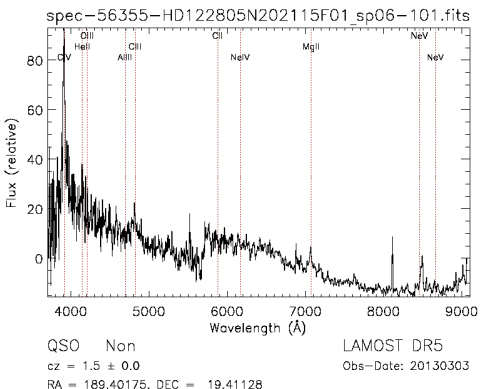

$\mathrm{J} 1250+1343$

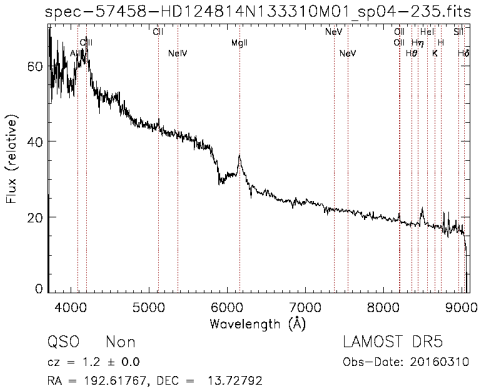

J1659+2629

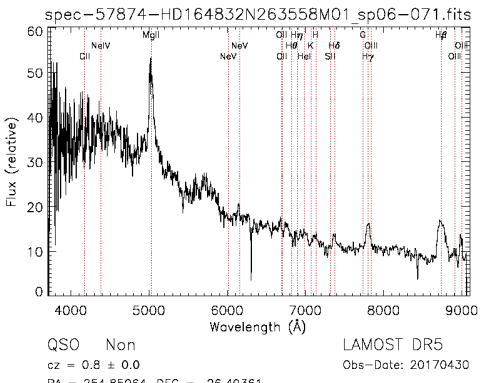

J2300+0337

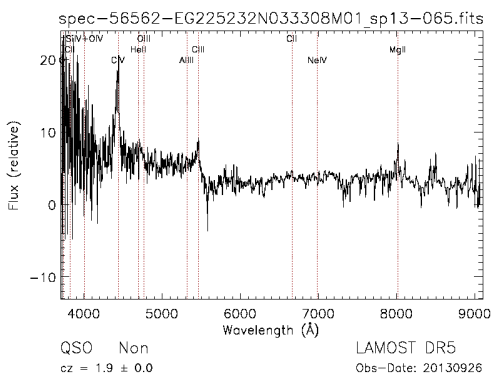

J2316+1618

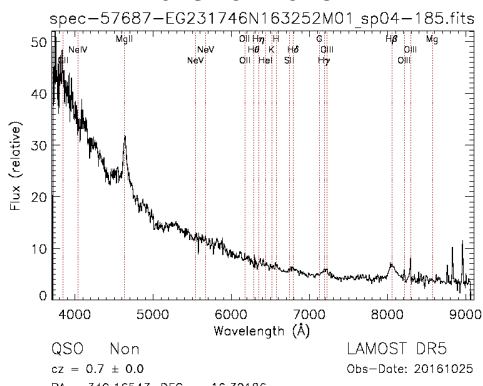

J2316+1618

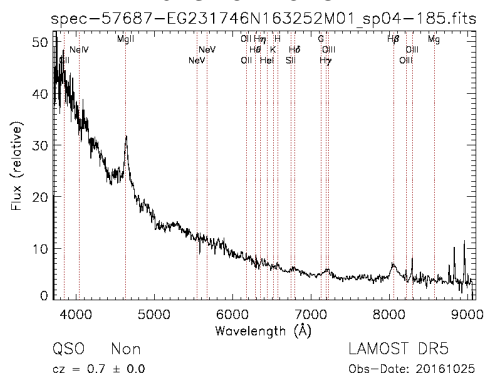

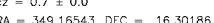

Fig. B.2. continued. 


\section{Appendix C: Optical spectra}

In this appendix we present tables collecting properties of $\mathrm{ABC}$ sources. In particular in Table C.1 we show the properties of the ABC sources found in other blazar catalogs (4FGL, 3HSP, WIBRaLS2 and KDEBLLACS), while in Table C.2 we present the infrared and optical properties of ABC sources.

Table C.1. ABC sources found in other blazar catalogs.

\begin{tabular}{|c|c|c|c|c|c|c|c|c|c|}
\hline Name ALMA & Redshift & Object type & Name 4FGL & Type 4FGL & $\begin{array}{l}\text { Name } \\
3 \text { HSP }\end{array}$ & Name WIBRaLS2 & $\begin{array}{l}\text { Type } \\
\text { WIBRaLS2 }\end{array}$ & $\begin{array}{l}\text { Class } \\
\text { WIBRaLS2 }\end{array}$ & $\begin{array}{l}\text { Name } \\
\text { KDEBLLACS }\end{array}$ \\
\hline J0008-3945 & 1.9 & RS & 4FGL J0008.0-3937 & $\mathrm{BCU}$ & & J000809.17-394522.8 & BZQ & $\mathrm{D}$ & \\
\hline $\mathrm{J} 0011+0823$ & 1.35 & QSO & & & & J001135.27+082355.5 & BZQ & $\mathrm{D}$ & \\
\hline J0011-8443 & 0.6 & RS & & & & J001145.90-844320.0 & BZQ & $\mathrm{C}$ & \\
\hline J0019-5641 & & RS & 4FGL J0019.2-5640 & $\mathrm{BCU}$ & & & & & \\
\hline J0024-0811 & 2.067 & QSO & & & & J002400.67-081109.8 & BZQ & $\mathrm{D}$ & \\
\hline J0024-4202 & 0.937 & QSO & & & & J002442.99-420203.9 & BZQ & $\mathrm{D}$ & \\
\hline J0024-6820 & 0.354 & AGN & 4FGL J0023.7-6820 & $\mathrm{BCU}$ & & J002406.72-682054.5 & BZQ & A & \\
\hline J0025-4803 & & RS & 4FGL J0025.7-4801 & $\mathrm{BCU}$ & & J002545.81-480355.1 & BZQ & $\mathrm{D}$ & \\
\hline $\mathrm{J} 0028+2000$ & 1.552 & QSO & 4FGL J0028.4+2001 & FSRQ & & J002829.81+200026.7 & BZQ & $\mathrm{D}$ & \\
\hline J0030-0211 & 2.1 & QSO & 4FGL J0030.6-0212 & $\mathrm{BCU}$ & & & & & \\
\hline
\end{tabular}

Notes. For each source we list the name (Name ALMA), the redshift (Redshift), the ABC source type (Object type), the name (Name 4FGL) and source class (Type 4FGL) as listed in the 4FGL catalog, the name of the source as listed in the 3HSP catalog (Name 3HSP), the name (Name WIBRaLS2) and blazar candidate class (Type WIBRaLS2) as listed in the WIBRaLS2 catalog, and the name of the source as listed in the KDEBLLACS catalog (Name KDEBLLACS). The full table is available in electronic form at the CDS.

Table C.2. Infrared and optical properties of ABC blazar candidates.

\begin{tabular}{|c|c|c|c|c|c|c|c|c|c|c|c|}
\hline Name ALMA & Redshift & Object type & Name WISE & Type WISE & Name LAMOST & $\begin{array}{l}\text { Type } \\
\text { OST }\end{array}$ & LAM- & Redshift LAMOST & Name SDSS & $\begin{array}{l}\text { Type } \\
\text { SDSS }\end{array}$ & Redshift SDSS \\
\hline J0002-2153 & & BL Lac & J000211.98-215310.0 & MIXED & & & & & & & \\
\hline J0006-2955 & 0.683 & QSO & J000601.12-295550.0 & BZU & & & & & & & \\
\hline J0008-3945 & 1.9 & RS & J000809.17-394522.8 & MIXED & & & & & & & \\
\hline J0011+0823 & 1.35 & QSO & $\mathrm{J} 001135.27+082355.5$ & MIXED & J001135.27+082355.6 & QSO & & $1.40175(0.0004989)$ & & & \\
\hline J0011-4105 & & RS & J001152.39-410545.1 & MIXED & & & & & & & \\
\hline J0011-8443 & 0.6 & RS & J001145.90-844320.0 & MIXED & & & & & & & \\
\hline J0024-0811 & 2.067 & QSO & J002400.67-081109.8 & BZQ & & & & & \multicolumn{2}{|c|}{ J002400.67-081110@2SO } & $2.071(0.00032)$ \\
\hline J0024-6820 & 0.354 & AGN & J002406.72-682054.5 & BZQ & & & & & & & \\
\hline J0024-4202 & 0.937 & QSO & J002442.99-420203.9 & BZQ & & & & & & & \\
\hline J0025+3919 & 1.946 & QSO & J002526.13+391935.6 & MIXED & & & & & & & \\
\hline
\end{tabular}

Notes. For each source we list the name (Name ALMA), the redshift (Redshift), the ABC source type (Object type), the name of WISE counterpart (Name WISE), the blazar candidate class obtained from WISE colors (Type WISE), the name of the LAMOST DR5 counterpart (Name LAMOST), the redshift as evaluated from the LAMOST spectrum (Redshift LAMOST) with the error in parenthesis, the name of the SDSS DR12 counterpart (Name SDSS), and the redshift as evaluated from the SDSS spectrum (Redshift SDSS) with the error in parenthesis. The full table is available in electronic form at the CDS. 